PNL-10514

UC-721

\title{
Assessment of Tank 241-C-106 Temperature Response Indications
}

L. L. Eyler

March 1995

Prepared for the U.S. Department of Energy under Contract DE-AC06-76RLO 1830

Pacific Northwest Laboratory

Operated for the U.S. Department of Energy by Battelle Memorial Institute 


\title{
DISCLAIMER
}

This report was prepared as an account of work sponsored by an agency of the United States Government. Neither the United States Government nor any agency thereof, nor Battelle Memorial Institute, nor any of their employees, makes any warranty, expressed or implied, or assumes any legal liability or responsibility for the accuracy, completeness, or usefulness of any information, apparatus, product, or process disclosed, or represents that its use would not infringe privately owned rights. Reference herein to any specific commercial product, process, or service by trade name, trademark, manufacturer, or otherwise does not necessarily constitute or imply its endorsement, recommendation, or favoring by the United States Government or any agency thereof, or Battelle Memorial Institute. The views and opinions of authors expressed herein do not necessarily state or reflect those of the United States Government or any agency thereof.

\author{
PACIFIC NORTHWEST LABORATORY \\ operated by \\ BATTELLE MEMORIAL INSTITUTE \\ for the \\ UNITED STATES DEPARTMENT OF ENERGY \\ under Contract DE-ACO6-76RLO 1830
}

Printed in the United States of America

Available to DOE and DOE contractors from the

Office of Scientific and Technical Information; P.O. Box 62, Oak Ridge, TN 37831; prices available from (615) 576-8401. FTS 626-8401.

Available to the public from the National Technical Information Service, U.S. Department of Commerce, 5285 Port Royal Rd., Springfield, VA 22161. 
PNL-10514

UC-721

\section{Assessment of Tank 241-C-106 Temperature Response Indications}

L. L. Eyler

March 1995

Prepared for the U.S. Department of Energy under Contract DE-AC06-76RLO 1830

Pacific Northwest Laboratory Richland, Washington 99352 


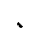

: 


\section{Contents}

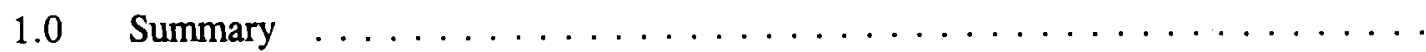

2.0 Identification of Significant Events, Historical Data, Observations, and Hypothesized Mechanism . . . . . . . . . . . . .

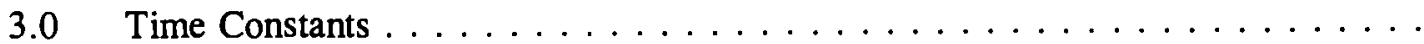

3.1 Estimate of Tank C-106 Time Constant . . . . . . . . . . . . .

3.2 Estimate of Tank C-106 Thermocouple Tree Time Constant . . . . . . . 3.2

4.0 Riser 14 Temperature Indications Analysis $\ldots \ldots \ldots \ldots \ldots \ldots \ldots \ldots \ldots$

4.1 Other Steady State Tank Modeling Results . . . . . . . . . . . . . . . 4.1

4.2 Thermocouple Tree Temperature Error Estimation . . . . . . . . . . . . . 4.2

4.3 Riser 14 TC4 Response to Water Additions . . . . . . . . . . . . 4.5

5.0 Effect of an Assumed Well Around Thermocouple Tree in Riser $14 \ldots \ldots . \ldots$

5.1 Steady State Temperature Distribution - Sludge in Contact with Pipe Wall . . . . . . . . . . . . . . . . . . .

5.2 Steady State Temperature Profile in Riser 14 Due to an

5.3 Quasi-Steady Response of Riser 14 Thermocouples to Convection in an Assumed Well . . . . . . . . . . . . . . . . . . . .

5.4 Transient Response of Riser 14 Thermocouples to Convection in an Assumed Well . . . . . . . . . . . . . . . . . . . .

5.5 Steady State Sludge Temperature Field Surrounding Riser 14 with an Assumed Well . . . . . . . . . . . . . . . . . . . .

5.6 Transient Temperature Response of Sludge Due to Assumed Closure of Well Gap . . . . . . . . . . . . . . . . 5.6

6.0 Temperature Response Due to Assumed Sludge Motion $\ldots \ldots \ldots \ldots . \ldots$

6.1 A Case for Sludge Motion - Narrative $\ldots \ldots \ldots \ldots \ldots \ldots \ldots$

$7.0 \quad$ References $\ldots \ldots \ldots \ldots \ldots \ldots \ldots \ldots \ldots \ldots \ldots \ldots \ldots \ldots$

Appendix: Supporting Information $\ldots \ldots \ldots \ldots \ldots \ldots \ldots \ldots \ldots \ldots \ldots \ldots$ 


\section{Figures}

$2.1 \quad$ Historical FIC Surface Level Indication - 1982 through August $1994 \ldots \ldots$

2.2 Historical FIC Surface Level Indication - 1992 through August $1994 \ldots \ldots$

2.3 Historical FIC Surface Level Indication $-1994 \ldots \ldots \ldots \ldots \ldots \ldots \ldots \ldots \ldots$

$2.4 \quad$ Historical Riser 14 Temperature Indication - 1982 through 1994, SACS Database $\quad 2.8$

2.5 Historical Riser 14 Temperature Indication - 1992 through August 1994,

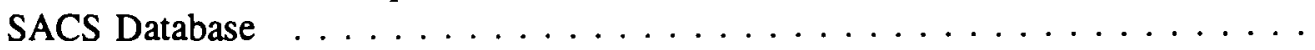

2.6 Historical Riser 14 Temperature Indication - 1994, SACS Database . . . . . . .

2.7 Historical Rise 8 Temperature Indication - 1982 through August 1994,

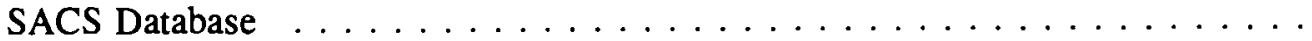

2.8 Historical Riser 8 Temperature Indication - 1992 through August 1994,

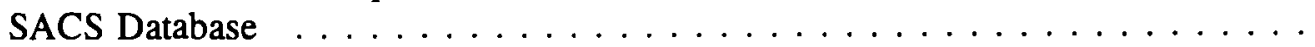

2.9 Historical Riser 8 Temperature Indication - 1994, SACS Database . . . . . . .

2.10 Comparison of SACS and TMACS Database of Temperatures for Riser 14,

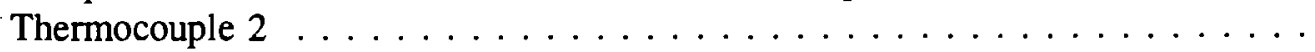

3.1 Comparison of Transient Temperatures in Riser $14 \mathrm{TC1}$ for Estimating Heat Up Time Response . . . . . . . . . . . . . . . . .

3.2 Decay of Maximum Evaporation Rate Following 1992 Ventilation Outage for Estimating Time Response $\ldots \ldots \ldots \ldots \ldots$

3.3 Response of Riser 14 to Step Change in Adjacent Sludge Temperature for Estimating Time Response $\ldots \ldots \ldots \ldots \ldots \ldots$

4.1 Riser 14 and Centerline Temperature Profiles $\ldots \ldots \ldots \ldots \ldots$

4.2 Schematic of Computational Domain for Investigation Thermocouple Response .

4.3 Riser 8 Thermocouple Tree Temperature Estimation Error for Axial Conduction

4.4 Riser 14 Thermocouple Tree Temperature Estimation Error for Axial Conduction with Colder Dome Space . . . . . . . . . . . . . . . . . .

4.5 Riser 8 Thermocouple Tree Temperature Estimation Error for Axial Conduction with Hotter Dome Space 
4.6 Response of Riser 14 TC4 to Water Additions After Process Test . . . . . . .

4.7 Comparison of Riser 14 and $8 \mathrm{TC} 3$, TC4, and TC5 Historical Temperatures, 1993 and 1994, SACS Database

4.8 Comparison of Riser 14 and 8 TC4 Response to Water Additions -

May to August 1994 , TMACS Database $\ldots \ldots \ldots \ldots \ldots$

5.1 Comparison of Riser 14 Predicted Steady State Temperature Distributions for Two Supernatant Temperatures $\ldots \ldots \ldots \ldots \ldots \ldots$

5.2 Comparison of Riser 14 Steady State Temperature Profiles for Assumed Gap Widths

5.3 Comparison of Riser 14 Quasi-Steady Temperature Response for

Assumed Gap Widths . . . . . . . . . . . . . . . . .

5.4 Riser 14 TC1 Response to an Assumed Convecting Well . . . . . . . . . . 5.10

5.5 Riser 14 TC2 Response to an Assumed Convecting Well . . . . . . . . . . 5.11

5.6 Riser 14 TC3 Response to an Assumed Convecting Well . . . . . . . . . . 5.12

5.7 Comparison of Response of Convecting Well to Observed Temperature

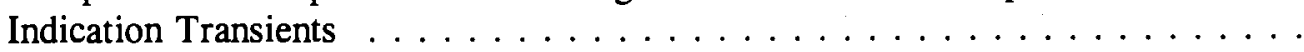

5.8 Steady State Temperature Distribution Around Riser 14 for an Assumed 1 -inch Convecting Gap Well $\ldots \ldots \ldots \ldots \ldots \ldots \ldots$

5.9 Steady State Temperature Distribution Around Riser 14 for an Assumed 2-inch Convecting Gap Well $\ldots \ldots \ldots \ldots \ldots \ldots$

5.10 Steady State Temperature Distribution Around Riser 14 for an Assumed 3-inch Convecting Gap Well $\ldots \ldots \ldots \ldots \ldots \ldots$

5.11 Steady State Temperature Distribution Around Riser 14 for an Assumed 6-inch Convecting Gap Well $\ldots \ldots \ldots \ldots \ldots \ldots$

5.12 Steady State Temperature Distribution Around Riser 14 for an Assumed Conical Shaped Convecting Gap Well . . . . . . . . . . . . . .

5.13 Comparison of Riser 14 TCs to Assumed Conduction Heat Up in Response to Loss of Convecting Gap Well . . . . . . . . . . . . . . .

6.1 Riser 14 Temperatures - SACS Database, January through August 1994 . . . . .

6.2 Riser 14 Temperatures - TMACS Database, Late June 1994, Showing Six Quasi-Periodic Oscillations . . . . . . . . . . . . . . . 


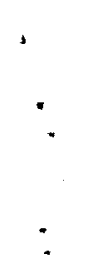




\subsection{Summary}

This report presents an assessment of waste tank 241-C-106 temperature response indications. The results are obtained through evaluation of historical data for FIC surface level data and temperature indication data from thermocouples in risers 8 and 14, contained in the SACS and TMACS databases. Computer analysis is used to augment observations and conclusions about hypothesized mechanisms present in the tank that could explain the data observations.

From the historical temperature indications of risers 8 and 14 (neglecting the ventilation outages), several general observational conclusions are drawn that support hypotheses explaining more recently observed behavior.

- Large variations have occurred historically in riser 8 and riser 14 indicated temperatures.

- Variability in riser 8 indicated temperatures decreased to the point of exhibiting characteristic annual environmental temperature changes in the 1991 time frame.

- Variability in riser 14 indicated temperatures exists up to approximately a point in time near the beginning of 1994.

- Riser 14 temperature variability essentially ceased in early 1994. This conclusion is compatible and consistent with a similar occurrence in riser 8 in 1991.

If these observational conclusions are considered, a consistent explanation for the most recent indicated temperature behavior can be found. To do so requires the following conclusions that:

- Evaporation was reduced during the time the sludge was uncovered in June 1994 which led to a storage of energy in the tank and development or expansion of a steam void.

- Sludge motion is or can be present in a limited region in the tank.

- A steam void is present to provide the sludge motion driving source.

- Riser 14 is surrounded by some nature of an enhanced cooling effect.

Steam void in the above context can refer to any nature of a region at saturation temperature where steam is formed.

After assuming that sludge convective motion is occurring in the sludge and that a steam void is the driving force, the larger, aperiodic, quasi-chaotic temperature oscillations occurring after August 6 are consistent. These temperature responses are probably due to lateral and vertical convective motions. They contrast with earlier temperature oscillations which are describable by cohesive or "elastic" sludge motions consistent with smaller amplitude lateral motions. The apparent net temperature decrease observed since August 1 is consistent with the fact that convective sludge motions are more efficient mixing motions. 
Several time constants and response times for this system are determined. The adiabatic temperature rise in the tank contents is estimated at $1^{\circ} \mathrm{F}$ per day. The time constant associated with the thermocouples in riser 14 is computed to be relatively fast, a matter of seconds, for an assumed step change in exterior surface temperature in direct contact with the tree's pipe wall. Correspondingly, the response time of an assumed convection well surrounding riser 14 is estimated at 10 to 20 minutes. The time response of the bottom of the sludge to a change in surface liquid temperature conditions is estimated at 6 to 8 days, based on indications of riser 14 temperatures. The surface temperature bounded heat-up time constant for the tank contents is determined from indicated temperature data during a period when effective thermal conduction is occurring and is estimated to be 17.5 days. The time constant associated with removal of stored energy by evaporative cooling based on the 1992 ventilation loss event is about 5.3 months. The multiplicity of time constants and response times of various features in this tank complicates specific identification of the controlling physical mechanisms.

The focus of this report is to specifically address temperature response indications. A much more detailed and specific evaluation of the evaporation issue is being addressed by other members of the C-106 Temperature Response Team.

Section 2 of this report summarizes historical data observations. Estimates of tank time constants are contained in Section 3. Section 4 contains analysis results of riser 14 temperature indications and Section 5 presents analysis results of enhanced cooling effects surrounding riser 14 . Section 6 presents a narrative explanation scenario of temperature response indications.

This results presented herein represent work conducted through mid-September 1994. 


\subsection{Identification of Significant Events, Historical Data Observations, and Hypothesized Mechanism}

The indicated temperature history of riser 14 thermocouples is used to identify significant events that affect the hypotheses and analyses conducted to understand tank C-106. Indicated temperature data for identification of these events are taken from the SACS and TMACS databases $^{\left({ }^{(a)}\right.}$.

FIC surface level data are presented in Figure 2.1 for the period from 1982 to August 1994. These data are expanded for 1992 through August 1994 in Figure 2.2 and expanded further for 1994 in Figure 2.3. Historically, the surface level in tank C-106 has been maintained between 75 and 79 inches. Water additions are indicated by the approximate step-changeupward indication of surface level. Evaporative loss of water is indicated by the slower, downward trend after each water addition. The events of particular interest in recent history are the 4-month ventilation outage during March to June 1992, a period of time when small water additions were made in 1993, and the process test which was started March 4, 1994, with the cessation of water additions and the re-starting of water addition on June 17, 1994. During this time, the surface level, as indicated by the FIC, dropped to 69.5 inches. It remained at 69.5 inches for a period of nearly a month because the FIC was resting on the sludge (which had been uncovered) or touching a "sludge weight" observed in videos. The significance of uncovering the sludge is that evaporation was probably reduced and hence energy was being stored in the tank. Removal of this excess energy is key to returning the tank to normal conditions ${ }^{(b)}$.

Indicated historical temperatures for TC1 through TC4 in riser 14 (1982 to the present) are presented in Figure 2.4. These data are expanded for 1992 through August 1994 in Figure 2.5 and further expanded for 1994 in Figure 2.6. The historical FIC surface level is also included in these figures; however, on the scale used in the figures, it is difficult to identify correspondence of indicated temperatures and surface level. Several events and observations in these data are of particular interest.

If temperatures that are indicated by riser 14 thermocouples are indeed correct, riser 14 temperatures during August 1994 are not largely different than historical data (Figure 2.4), even though the frequencies of data entry are quite different. Unfortunately, these data are not reported for the time the ventilation system was inoperative in 1992. In Figure 2.5 data,

(a) SACS database temperature data were obtained from Paul Whitney/PNL in the form of ASCII computer files. Only limited effort was made to "clean up" the data to remove obviously incorrect information. The SACS database contains data at relatively large time intervals and may not be all the data available from tank C-106. Data for the most recent time (period of May 1994 through August 1994) was from the TMACS database and was more extensive.

(b) Normal conditions for this tank are, as yet, undefined. 
the temperatures in riser 14 show oscillations during 1993 . Nearly coincidental with the beginning of the process test in March 1994, these oscillations stopped and temperature indications contained much less variability. This observation may be significant in defining normal conditions or concluding when normal conditions have again been attained.

The riser 8 historical temperature indications are presented in Figures 2.7 to 2.9 for similar time spans. Although some obviously inaccurate indicated temperatures are found in these data, they indicate that, historically, riser 8 temperatures varied markedly. In the most recent three years (Figure 2.8) the temperature rise during the 1992 ventilation outage is obvious, as are annual cyclic temperature variations. In looking at these data, it is very difficult to identify any temperature variations in riser 8 that resulted directly from the process test. The variation in the March to June 1994 time frame of the process test is consistent with ambient environmental changes.

From the historical temperature indications of risers 8 and 14 (neglecting the ventilation outages for the moment), several general observational conclusions are drawn that support the hypotheses made to explain more recent observed behavior.

- Large variations have occurred historically in both riser 8 and riser 14 indicated temperature.

- Variability in riser 8 indicated temperatures decreased to the point of exhibiting characteristic annual environmental temperature changes in the 1991 time frame. This fact indicates that riser 8 is in a region that has developed into a compacted or consolidated (meaning essentially non-convecting) sludge with some degree of strength and the thermal field around riser 8 can be described by effective conduction heat transfer.

- Variability in the riser 14 indicated temperatures exists up to approximately a point in time near the beginning of 1994 . This observation, coupled with the observation that riser 14 temperatures are indicated to be less than those of riser 8 , supports the observation that some sort of enhanced cooling is present in the vicinity of riser 14 . This enhanced cooling could be due to one of several reasons, but the most logical is that convective sludge motion was occurring. Even a vertical plane circulatory flow, that is upward at the tank center and downward in the vicinity of riser 14, could exhibit such a local cooling effect.

- Riser 14 temperature variability essentially ceased in early 1994 . This observation is compatible and consistent with a similar occurrence in riser 8 . In this case, the indication that riser 14 is cooler than riser 8 would be supported if a local convectivewell cooling effect developed after cessation of sludge convective motions. A possible mechanism for such an occurrence can be hypothesized, based on small amplitude 
movement of the tree. Such movements may be caused by vibrations or by a flexure movement induced by sludge motion, if the bottom of the tree is "pinned" in the hard $\operatorname{pan}^{(a)}$.

The most logical driving source for convective sludge motions is a steam void. The presence of such a void has previously been indicated by several independent effective conductivity analyses (Bander 1994) and by analyses conducted with a multiphase computer code (Thurgood et al. 1994).

If these observational conclusions are considered, a consistent explanation for the most recent indicated temperature behavior can be formed. To do so requires the following conclusions:

- Evaporation was reduced during the time the sludge was uncovered which led to a storage of energy in the tank.

- Sludge motion can be or is present in a limited region in the tank.

- A steam void can be or is present to provide the sludge motion driving source.

- Riser 14 is surrounded by some nature of an enhanced cooling effect. Any hypothesized enhanced cooling effect must account for historically observed increases in temperature in TC3 of riser 14 with each water addition.

Indications of these effects are identified in Figure 2.10 where riser 14 temperature data from the SACS database and the TMACS database are compared. Note the SACS database contains data at less frequent time intervals than does the TMACS database. Examination of the SACS data alone, in the period from May 1994 to the present, does not present some of the most relevant features of the indicated temperature response. For that reason, the TMACS data are used throughout the rest of this report. The data that was available for the present analysis was in a format where time was measured in days referenced to May 9 , 1994 as zero.

The first indication of sludge motion evident in the indicated temperature is the increase in temperature in TC2 in the June 9-12 (day 29-32) time frame. This increase in temperature is significant, about $25^{\circ} \mathrm{F}$. The indication appears to be reversible which is an indication that hotter sludge moved past the thermocouple tree and subsequently moved back. Time constants associated with this motion are examined subsequently. The other feature of this temperature increase is that it is largely indicated only in TC2. TC1 shows a small response at the same time, and TC3 has little or no variation.

(a) Memo D. L. Lessor to L. L. Eyler, dated September 9, 1994. "Separation Phenomena Around Columns in Tank Waste." Included in the Appendix. 
The first water addition after the process test was on June 17 (day 39). This addition is associated with an increase in TC2 temperature of about $10^{\circ} \mathrm{F}$. It is interesting to note that if the riser 14 data in Figure 2.5 is overlaid with the FIC data in Figure 2.2, a direct correspondence is present between an increase of $10^{\circ} \mathrm{F}$ in TC2 temperature and each water addition. After the next water additions of June 21, 22, and 23, the temperature response is indicative of a conduction heat transfer heat-up process.

The next indication of sludge motion is the six quasi-periodic temperature fluctuations occurring between about June 26 through July 3 (days 48 through 55). The end of these fluctuations corresponds directly with the addition of the 14,000 gallons of water starting on July 3. The driving force for these quasi-periodic oscillations can be coincided with hypothesized growth and collapse of some fraction of the steam void inducing some sort of elastic sludge motion. In this context, elastic sludge motion may be of any nature that maintains the local sludge integrity or cohesiveness. Any of a number of non-Newtonian fluid or sludge rheologies could exhibit this character in a slow, small amplitude, movement. The indicated temperature oscillations are sufficiently consistent to conclude that these elastic sludge motions are primarily of a horizontal or lateral nature. This conclusion results from the comparison of responses of TC1, TC2, and TC3 as being largely in phase and of the same order of magnitude. If it is hypothesized the steam void collapsed as a result of the increased hydrostatic pressure gradient corresponding to the added water level, the relatively quiescent period up to about the first of August could be a conduction heat-up period, at least in the region surrounding riser 14.

Near the first of August, the steam void had reformed or re-grown to a volume sufficient to again begin to induce motion in the sludge. The consistency in these hypotheses is that repeated effects of steam void growth and collapse leads to a gradual breakdown of sludge strength over a larger and larger region until convective sludge motion caused by the steam void encompasses a region out to the riser 14 radial position. During all this time, riser 8 has little or no sense of this activity because it exists in a non-convective sludge region. No temperature oscillations of noticeable magnitude are present in riser 8 temperatures beyond those typical of normal historical trends with environmental temperature changes.

After a convective motion is assumed to occur in the sludge, the large, aperiodic, quasichaotic-appearing temperature oscillations after August 6 (day 89) are consistent with steamvoid-driven sludge convection. These sludge convective motions are more likely to be combined lateral and vertical motions in contrast to the previously described elastic motions. The apparent net temperature decrease observed is also consistent with sludge motion because convective sludge motion would probably be a more efficient mixing mechanism (albeit on a rather slow time scale).

Together with described and hypothesized physical mechanisms, certain analyses were conducted in an attempt to determine whether the time scales and magnitudes of temperature response are consistent. The remainder of this report presents results of analyses conducted to investigate these effects. 


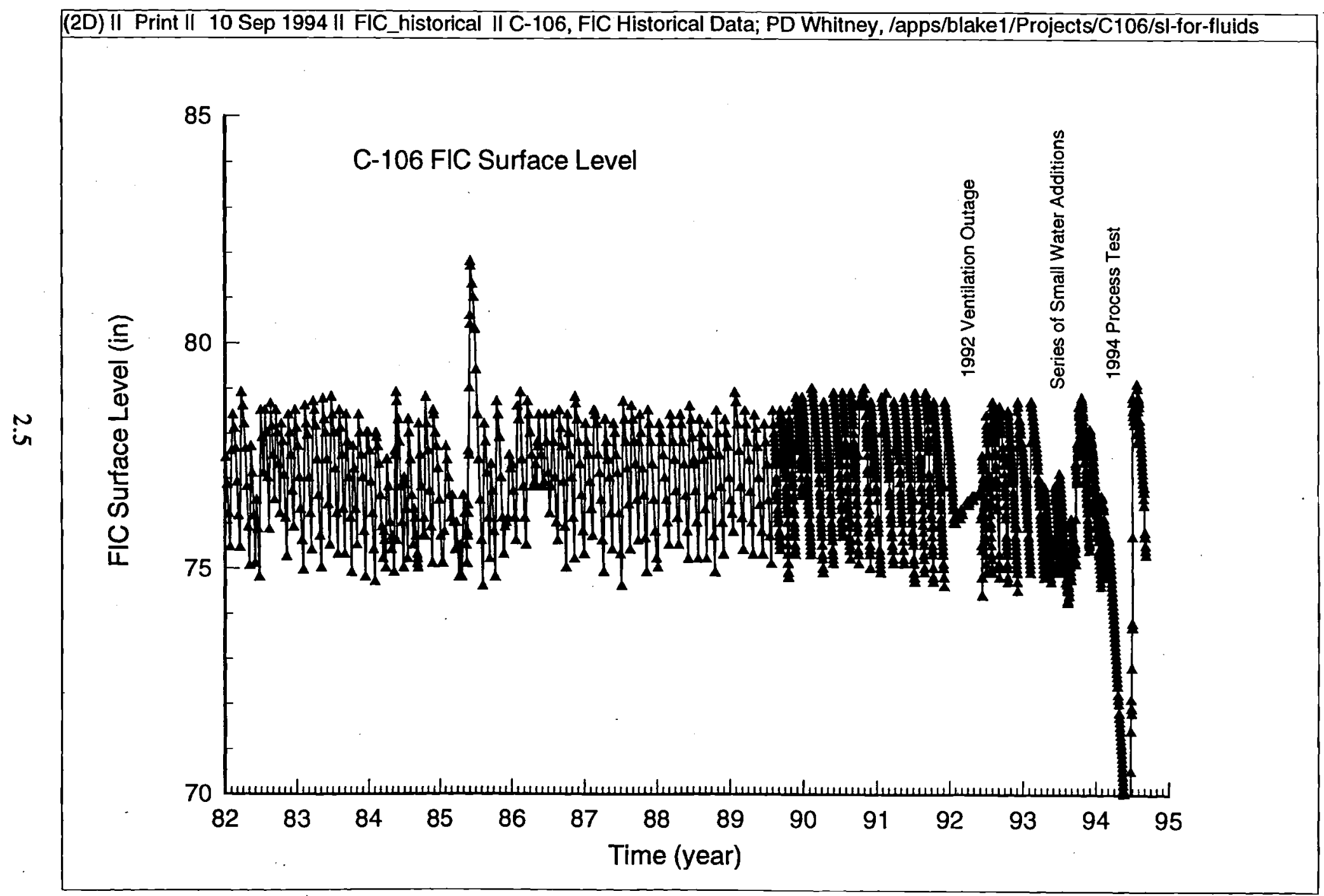

Figure 2.1. Historical FIC Surface Level Indication - 1982 through August 1994 


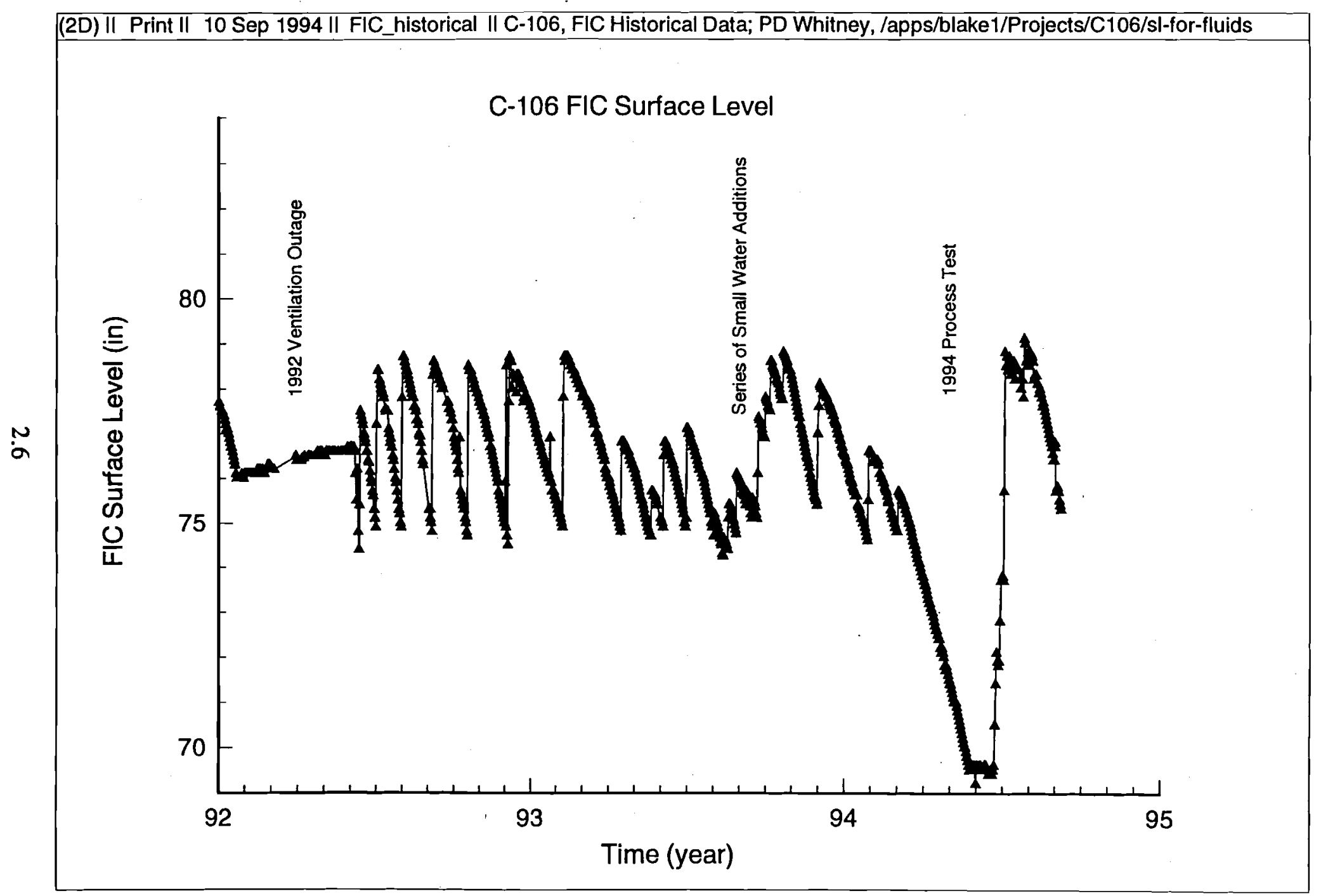

Figure 2.2. Historical FIC Surface Level Indication - 1992 Through August 1994 


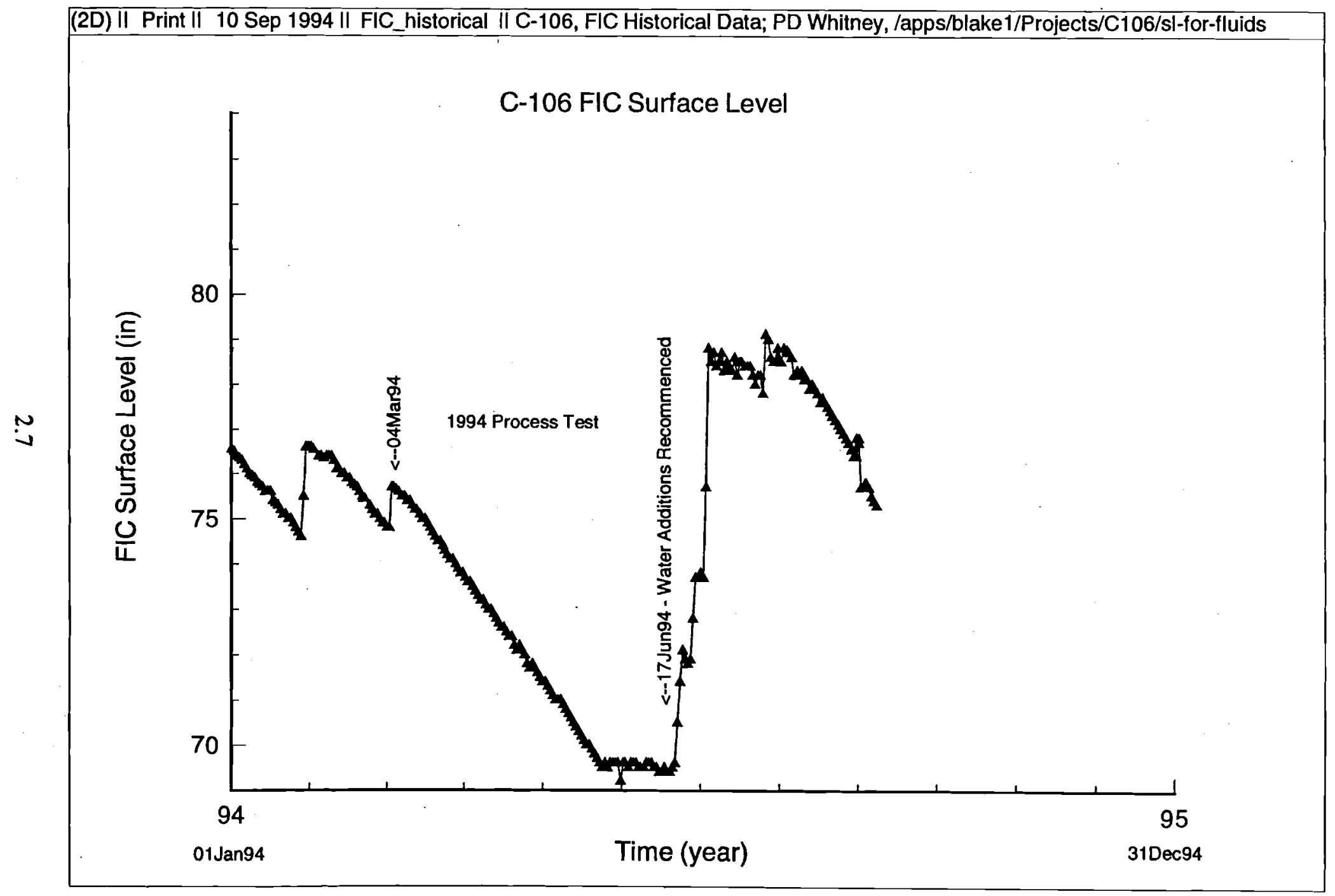

Figure 2.3. Historical FIC Surface Level Indication - 1994 


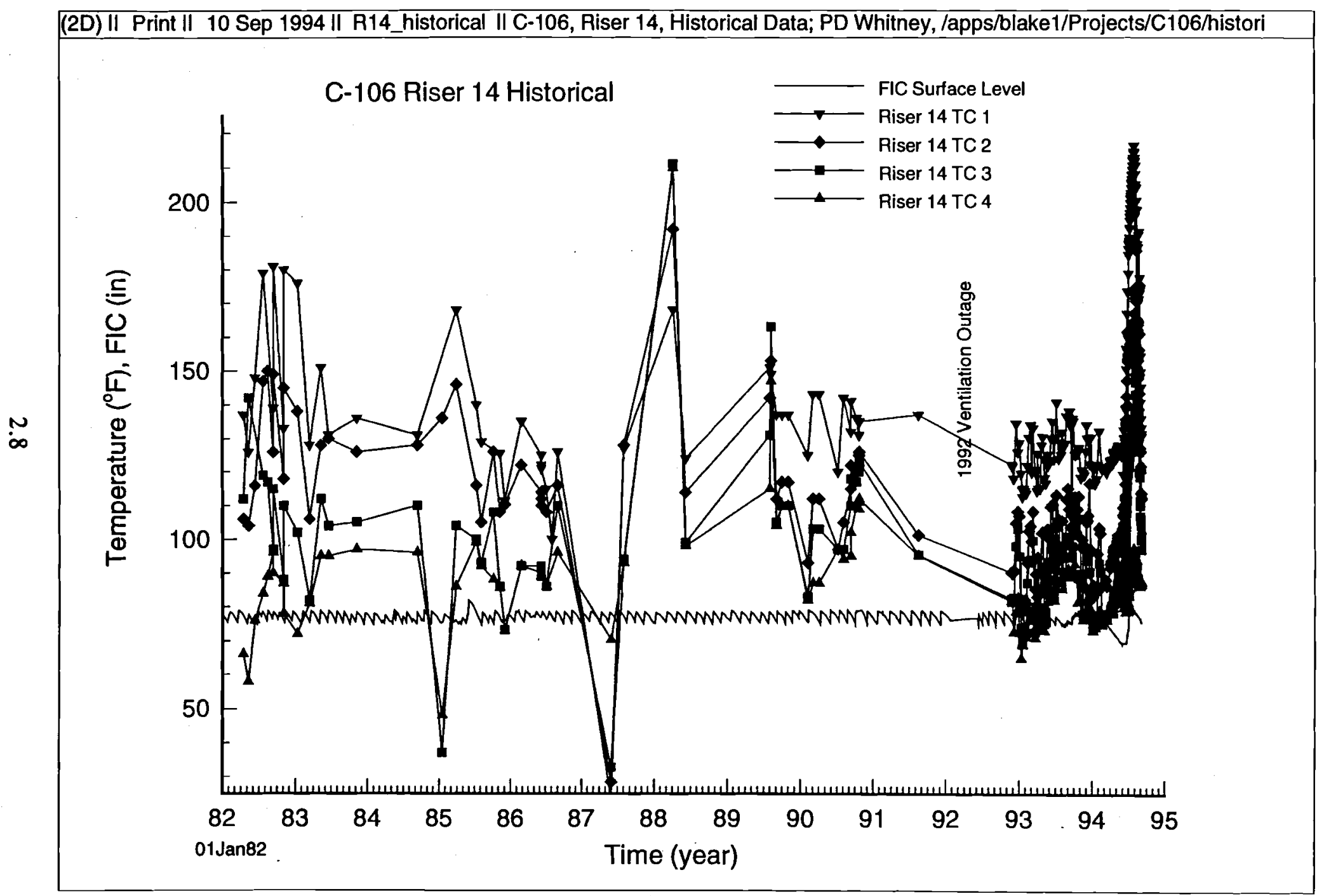

Figure 2.4. Historical Riser 14 Temperature Indication - 1982 through August 1994, SACS Database 


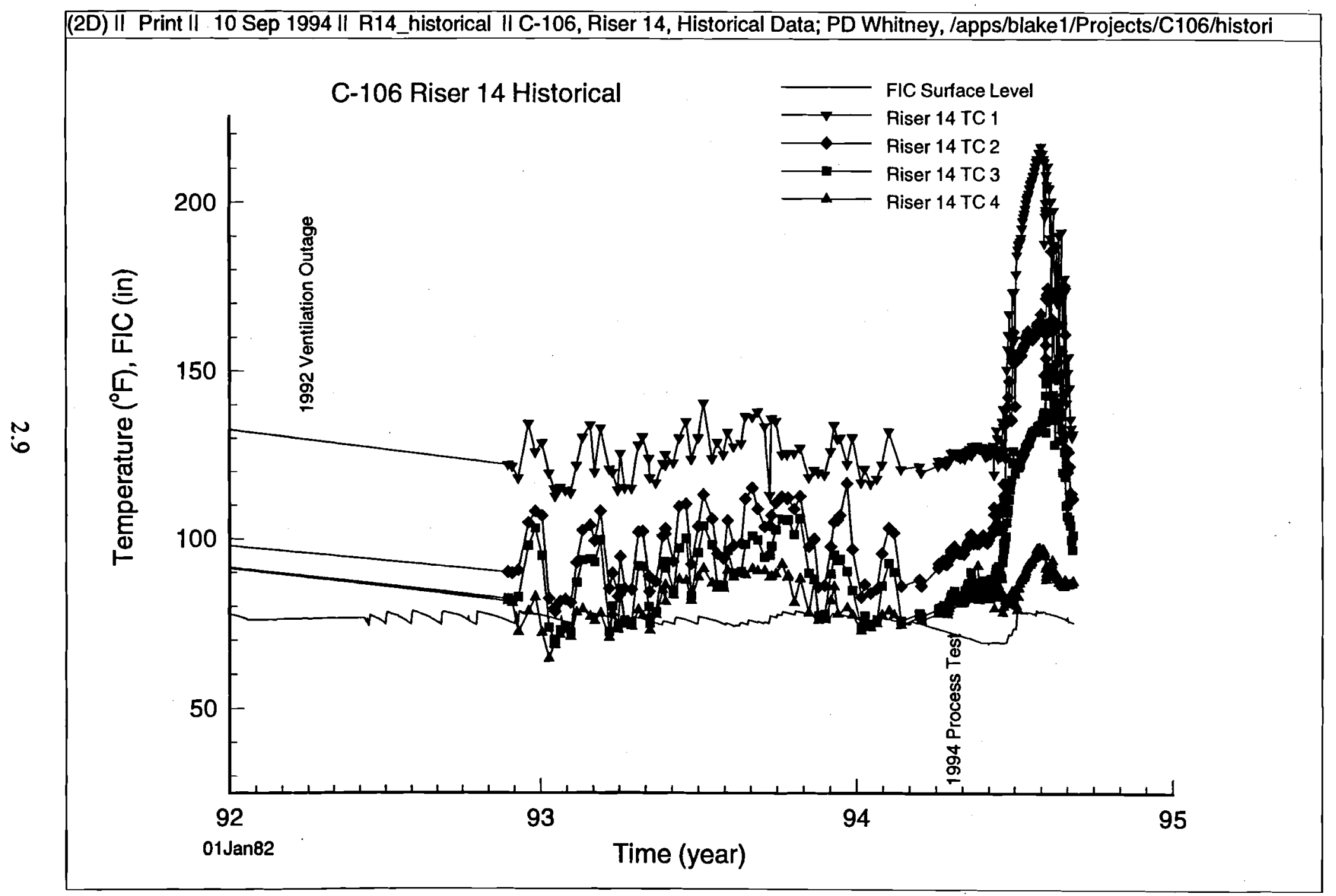

Figure 2.5. Historical Riser 14 Temperature Indication - 1992 through August 1994, SACS Database 


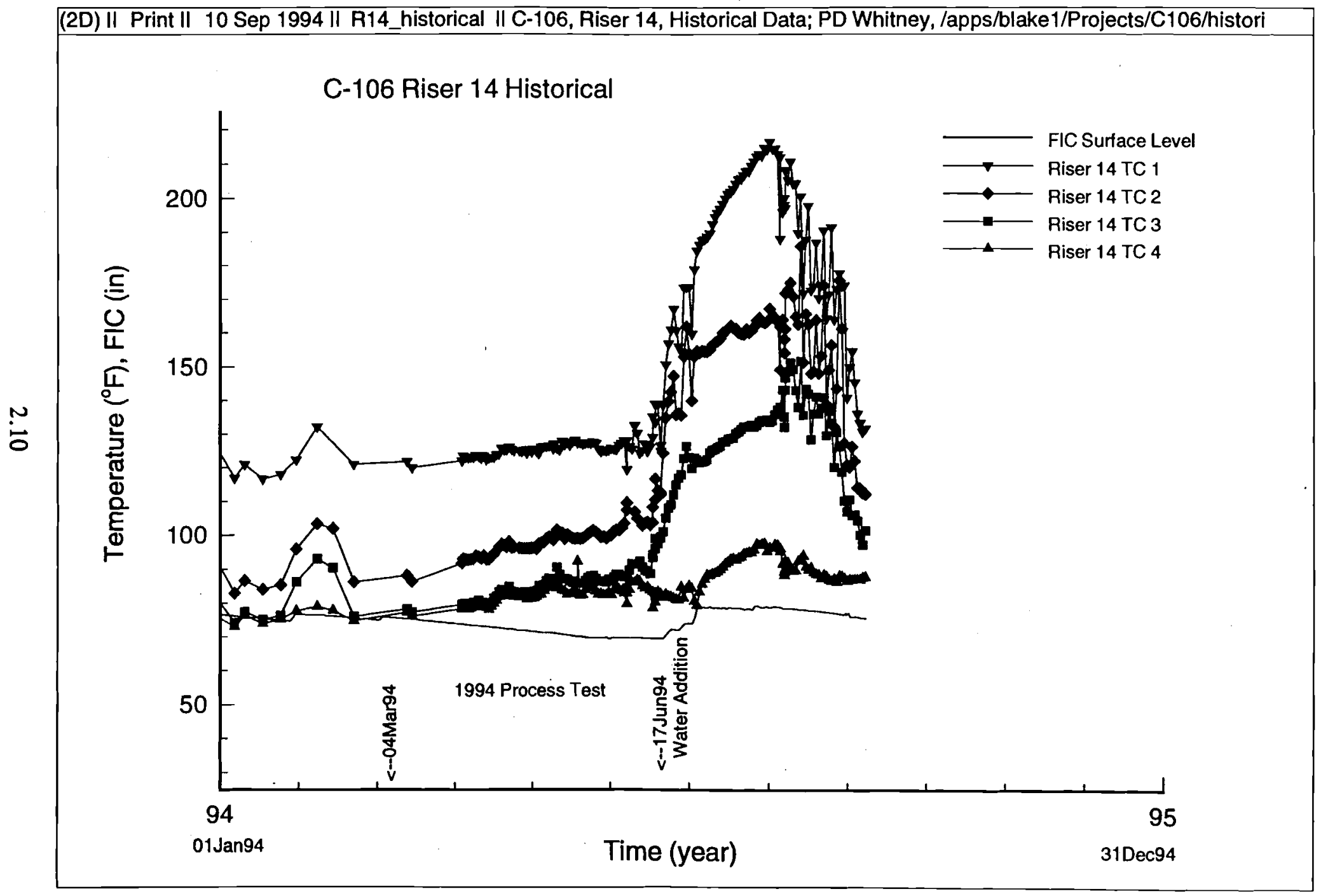

Figure 2.6. Historical Riser 14 Temperature Indication - 1994, SACS Database 


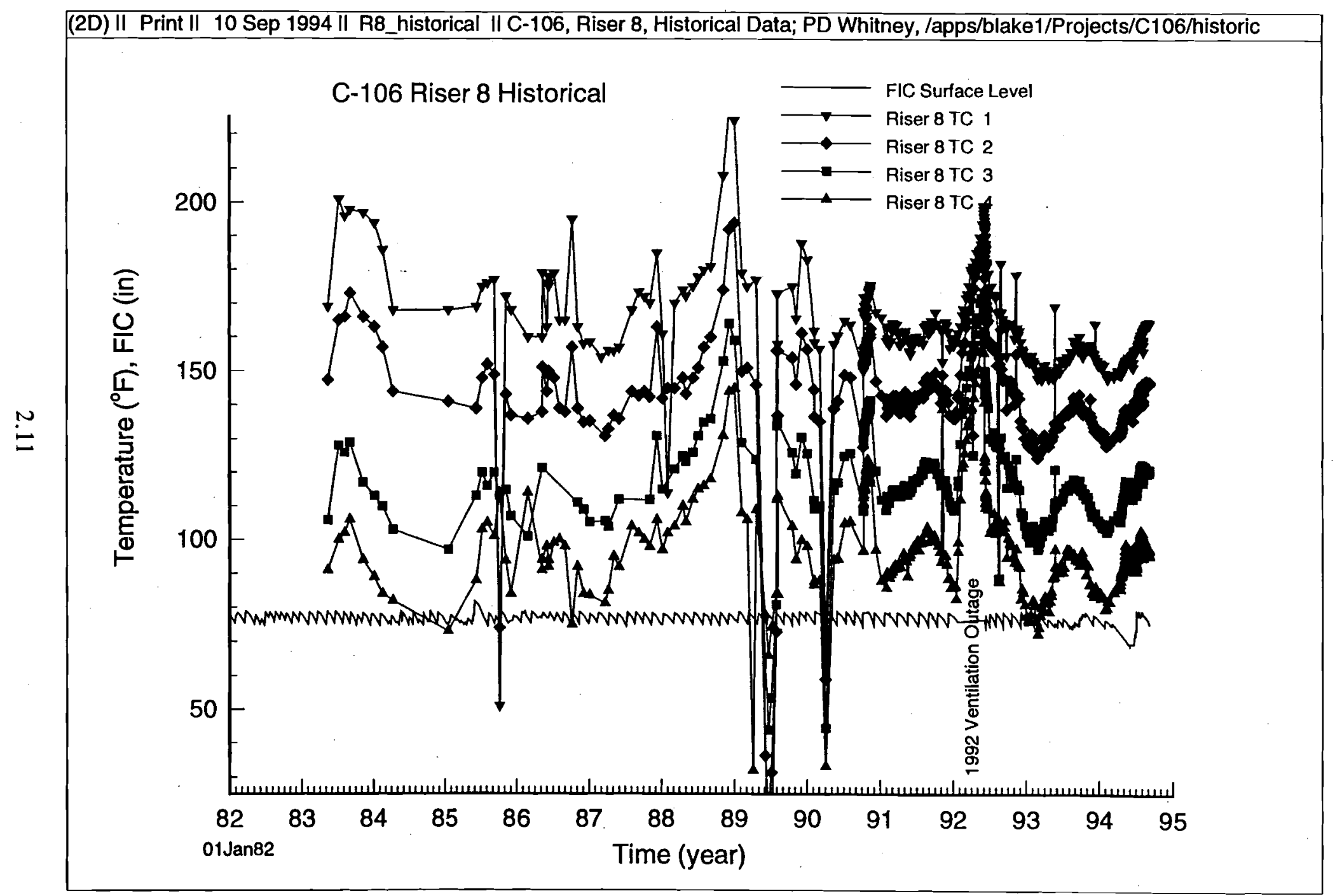

Figure 2.7. Historical Riser 8 Temperature Indication - 1982 through August 1994, SACS Database 


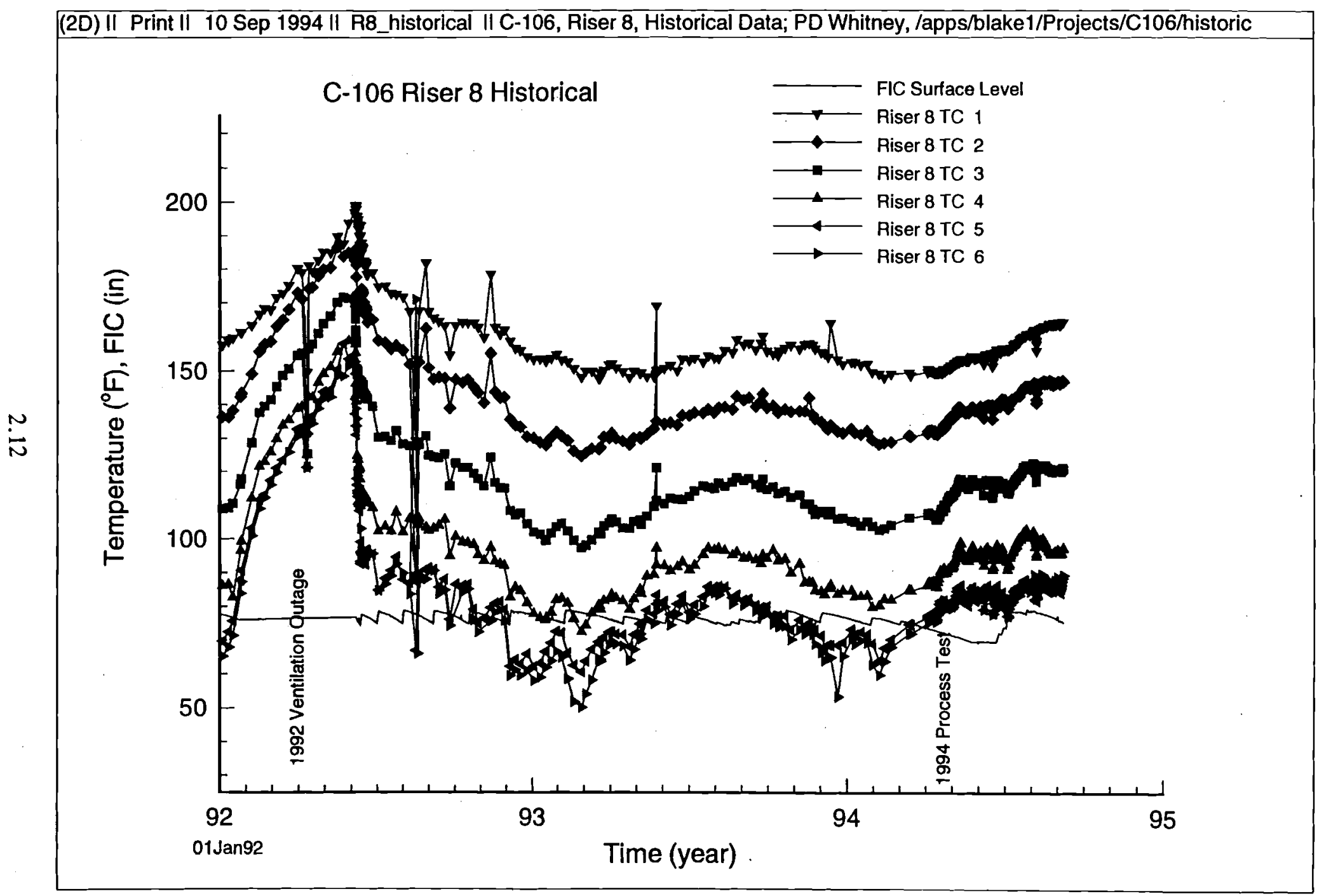

Figure 2.8. Historical Riser 8 Temperature Indication - 1992 through August 1994, SACS Database 


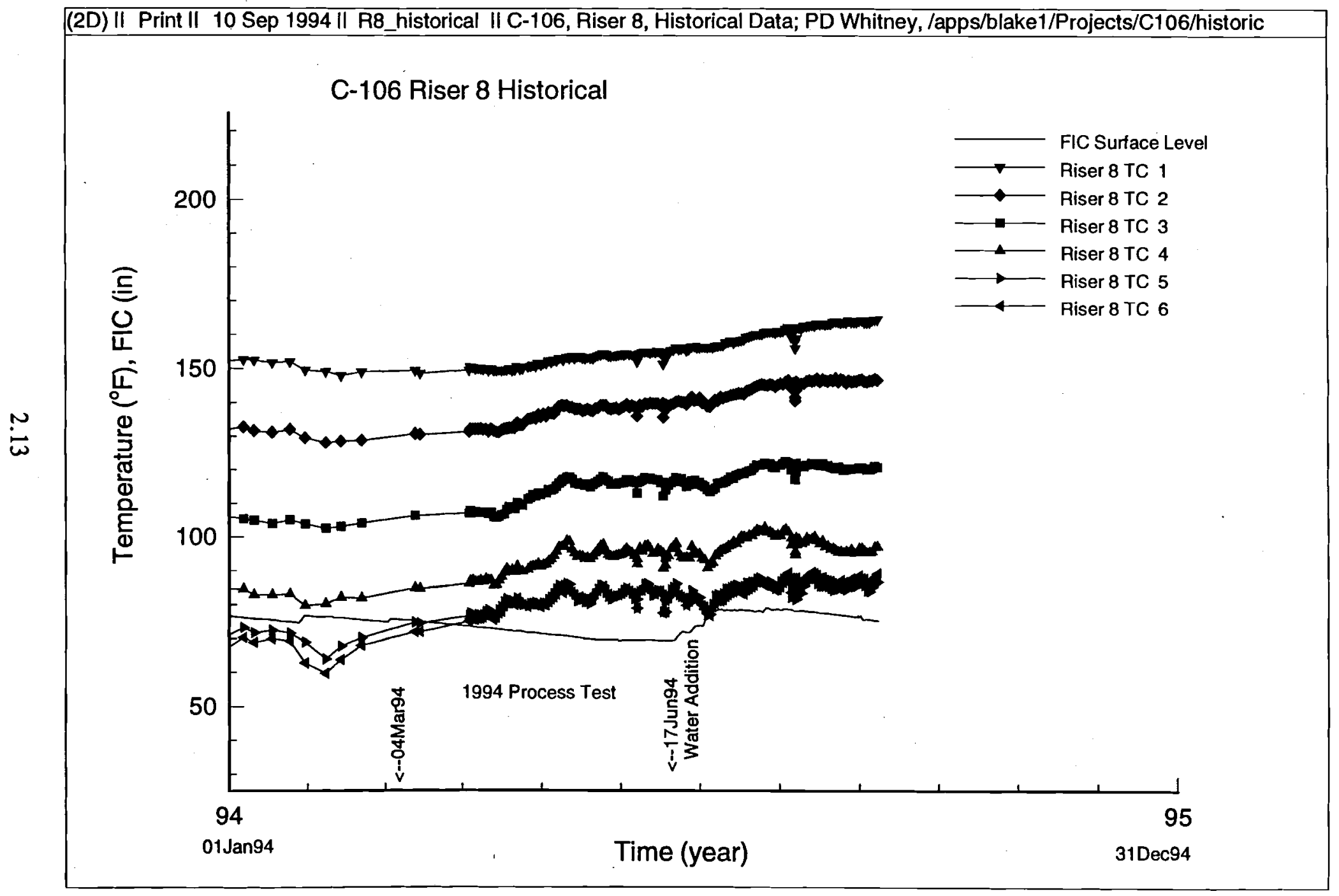

Figure 2.9. Historical Riser 8 Temperature Indication - 1994, SACS Database 


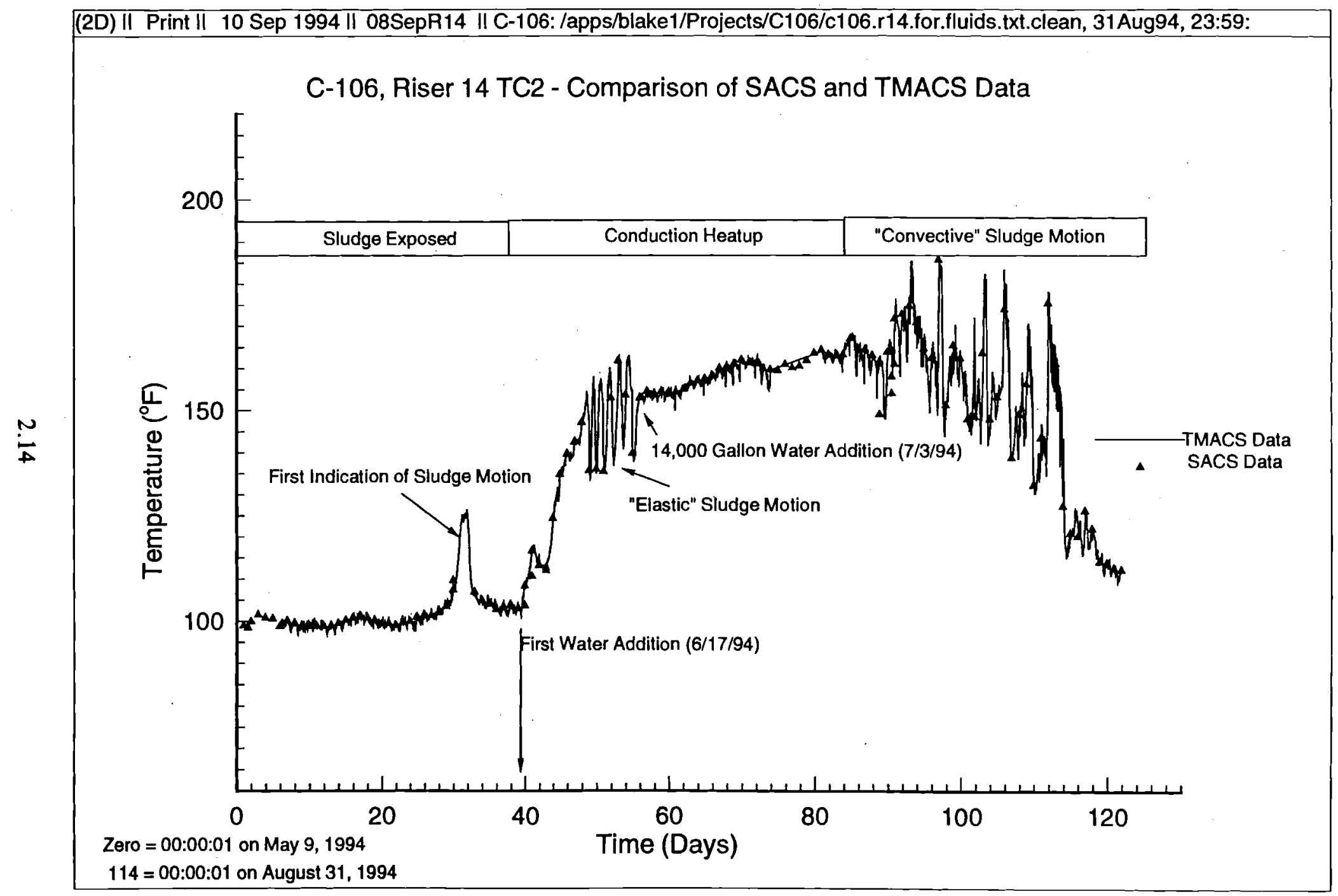

Figure 2.10. Comparison of SACS and TMACS Database of Temperatures for Riser 14, Thermocouple 2 


\subsection{Time Constants}

\subsection{Estimate of Tank C-106 Time Constant}

Estimates of tank C-106 time constant were made in two ways.

- Using the temperature data indicated by TC1 in riser 14 during the time when the data indicated (by eye) a decaying exponential growth.

- Using the maximum of evaporation rate curves for the first six water addition cycles after the resumption of ventilation at the end of the ventilation outage in 1992.

Method 1. During the time period of about July 9 to about July 24, 1994, the indicated temperature for TC1 in riser 14 exhibit (by eye) a decaying exponential growth that appeared to be not too atypical of a bounded lumped parameter heat up. Thus, a curve fit was made of the indicated temperature of the form

$$
\mathrm{T}=\mathrm{T}_{\mathrm{o}}+\Delta \mathrm{T}(1-\mathrm{EXP}(-\mathrm{A}(\mathrm{t}-28))
$$

where

$$
\begin{array}{ll}
\mathrm{T} & =\text { Temperature, }{ }^{\circ} \mathrm{F} \\
\mathrm{t} & =\text { Time [after } 21 \mathrm{Jun} 94(\mathrm{t}=5 \text { is June 26)], in days } \\
\mathrm{T}_{\mathrm{o}} & =\text { Reference temperature at } \mathrm{t}=28=\text { July } 18, \text { a curve fit constant, }{ }^{\circ} \mathrm{F} \\
\Delta \mathrm{T} & =\text { Temperature increase above reference temperature, a curve fit constant, }{ }^{\circ} \mathrm{F} \\
\mathrm{A} & =\text { Curve fit constant, (1/days) }
\end{array}
$$

In this form, 1/A is a time constant corresponding to reaching a $1 / \mathrm{e}$ value of the expected temperature increase. Several curve fits were done using several time spans of indicated temperature. A typical set of constants are:

$$
\begin{aligned}
\mathrm{T}_{\mathrm{o}} & =203.3^{\circ} \mathrm{F} \\
\Delta \mathrm{T} & =17.9^{\circ} \mathrm{F} \\
\mathrm{A} & =0.057 \text { days }^{-1}
\end{aligned}
$$

The time constant is then

$$
\tau=1 / \mathrm{A}=17.5 \text { days }
$$


The curve fit is presented in Figure 3.1 along with TC1 data and a transient PTHERM calculation result done by Bander ${ }^{(a)}$. Note the curve fit was only for data over the time from July 9 to July 24, 1994. Extension of the curve fit back to June 21, 1994 (day 0 in Figure 3.1) shows very good agreement. This observation further supports the conclusion of a conduction heat-up process occurring (at least locally).

Method 2. The ventilation system was inoperative during a 4-month period of time from January 25 to June 7, 1992. During this time, the temperatures in the sludge in the tank showed a marked increase because evaporative cooling was significantly reduced by the loss of ventilation flow in the dome space. Subsequent to restart of the ventilation, normal water addition cycles were resumed. During the first four or five water addition cycles, evaporation rates, as indicated by liquid level decrease with time, were significantly greater than normal. These increased rates were a consequence of the increased liquid pool surface temperature. The peak evaporation rate for each water addition cycle showed a characteristic decrease as the excess energy stored in the tank during the ventilation outage was removed. The peak evaporation rates were curve fit with a exponential decay of the form

$$
\mathrm{E}_{\mathrm{r}}=\mathrm{E}_{\mathrm{r}, \mathrm{n}}+\left(1+\left(\mathrm{E}_{\mathrm{r}, \mathrm{o}} / \mathrm{E}_{\mathrm{r}, \mathrm{n}}-1\right) * \operatorname{EXP}(-\mathrm{At})\right)
$$

where

$$
\begin{array}{ll}
\mathrm{E}_{\mathrm{r}} & =\text { Peak evaporation rate, gal/day } \\
\mathrm{t} & =\text { Time, months } \\
\mathrm{E}_{\mathrm{r}, \mathrm{n}} & =\text { Nominal equilibrium evaporation rate, assumed equal to } 189 \mathrm{gal} / \mathrm{day} \\
\mathrm{E}_{\mathrm{r}, \mathrm{o}} & =\text { Initial peak evaporation rate, a curve fit constant, gal/day } \\
\mathrm{A} & =\text { Curve fit constant, } 1 / \text { months }
\end{array}
$$

From the curve fit, the following constants were determined

$$
\begin{aligned}
& \mathrm{E}_{\mathrm{r}, \mathrm{o}}=581 \mathrm{gal} / \text { day } \\
& \mathrm{A}=0.190 / \text { months }
\end{aligned}
$$

and the time constant is thus $\tau=1 / \mathrm{A}=5.3$ months. The curve fit and data used are presented in Figure 3.2. By this approach, the time constant of the total tank system appears to be very long.

\subsection{Estimate of Tank C-106 Thermocouple Tree Time Constant}

Estimates of tank C-106 thermocouple tree time constant were made using a computed sludge temperature distribution directly adjacent to the thermocouple tree pipe wall as a step change boundary condition at the pipe wall. These estimates were done for the tree in riser 14 and

(a) T. J. Bander/WHC, personal communication of data presented by Bander (1994). 
assumed the pipe interior was adiabatic, filled with convecting air, filled with convecting water, and filled with non-convecting water.

A schematic of the tree in riser 14 is presented in the Appendix. It consists of a 2-inch Schedule 40 carbon steel pipe. Properties assumed for the steel are as follows.

$\begin{array}{ll}\text { Thermal conductivity } & =25 \mathrm{Btu} / \mathrm{hr}-\mathrm{ft}-\mathrm{F} \\ \text { Specific heat } & =0.1 \mathrm{Btu} / \mathrm{lbm}-\mathrm{F} \\ \text { Density } & =491 \mathrm{lbm} / \mathrm{ft}^{3}\end{array}$

These values are from Marks (1978).

Figure 3.3 presents the transient temperature results at three thermocouple locations. The locations are at distances approximately equal to $\mathrm{TC} 1, \mathrm{TC} 2$, and $\mathrm{TC} 3$ positions in the tree. Relative to FIC surface level indication, these are positions are 4, 28, and 52 inches. The outer surface temperature was specified by a pre-computed steady state temperature distribution in the adjacent sludge. The thermocouple tree was initialized to a temperature of $80^{\circ} \mathrm{F}$. The transient response of the tree was computed with a conduction model using the TEMPEST computer code. The transient response curves in the figure are temperatures at the interior pipe wall surface approximately where thermocouple junctions are located.

Note that for all four assumptions regarding the interior of the thermocouple tree, the initial response is very fast. For the adiabatic and convecting air cases, $99 \%$ of the boundary temperature is reached within five seconds. For the water-filled cases, the initial response is also very fast, but the asymptotic approach to the boundary temperature is quite slow. This response is because the assumed water-filling has a specific heat nearly 10 times that of steel. For the convecting water-filled case, the additional cooling effect is computed to be significant. This interior convecting cooling results in a $4^{\circ} \mathrm{F}$ temperature drop across the pipe wall. No conclusion is made that implies the thermocouple tree is liquid-filled. The 1/e time constant for all four cases is a matter of seconds.

Taking the derivative of Eq. (1) around $t=0(\equiv 7 / 19)$, the time rate of temperature increase (slope) is

$$
\mathrm{dT} / \mathrm{dt}=17.88 * 0.05706=1.02^{\circ} \mathrm{F} / \mathrm{day}
$$

Furthermore, if an adiabatic heat-up rate is calculated from

$$
\mathrm{dT} / \mathrm{dt}=\mathrm{Q} / \rho \mathrm{C}_{\mathrm{p}} \mathrm{V}
$$


where

$$
\begin{aligned}
& \mathrm{Q}=\text { Total heat rate }(=100,000 \mathrm{Btu} / \mathrm{hr}) \\
& \rho=\text { Average sludge density }\left(=1.43 \mathrm{SG}=89.2 \mathrm{lbm} / \mathrm{ft}^{3}\right) \\
& \mathrm{C}_{\mathrm{p}}=\text { Average specific heat }(=1.0 \mathrm{Btu} / \mathrm{lbm}-\mathrm{F}) \\
& \mathrm{V}=\text { Total sludge volume }\left(=26,500 \mathrm{ft}^{3}\right)
\end{aligned}
$$

then $\mathrm{dT} / \mathrm{dt}=1.01^{\circ} \mathrm{F} /$ day. Although this number is remarkably close to the curve fit data slope around July 19, it is only approximately indicative that heat-up rates around the thermocouples in riser 14 are representative of an average heat-up rate of the sludge material. However, this number does indicate the hole around riser 14 may well have filled in due to a sludge redistribution. It also further supports the conclusion that temperatures observed in riser 14, during the conduction heat-up time period, are representative of local sludge temperatures. 


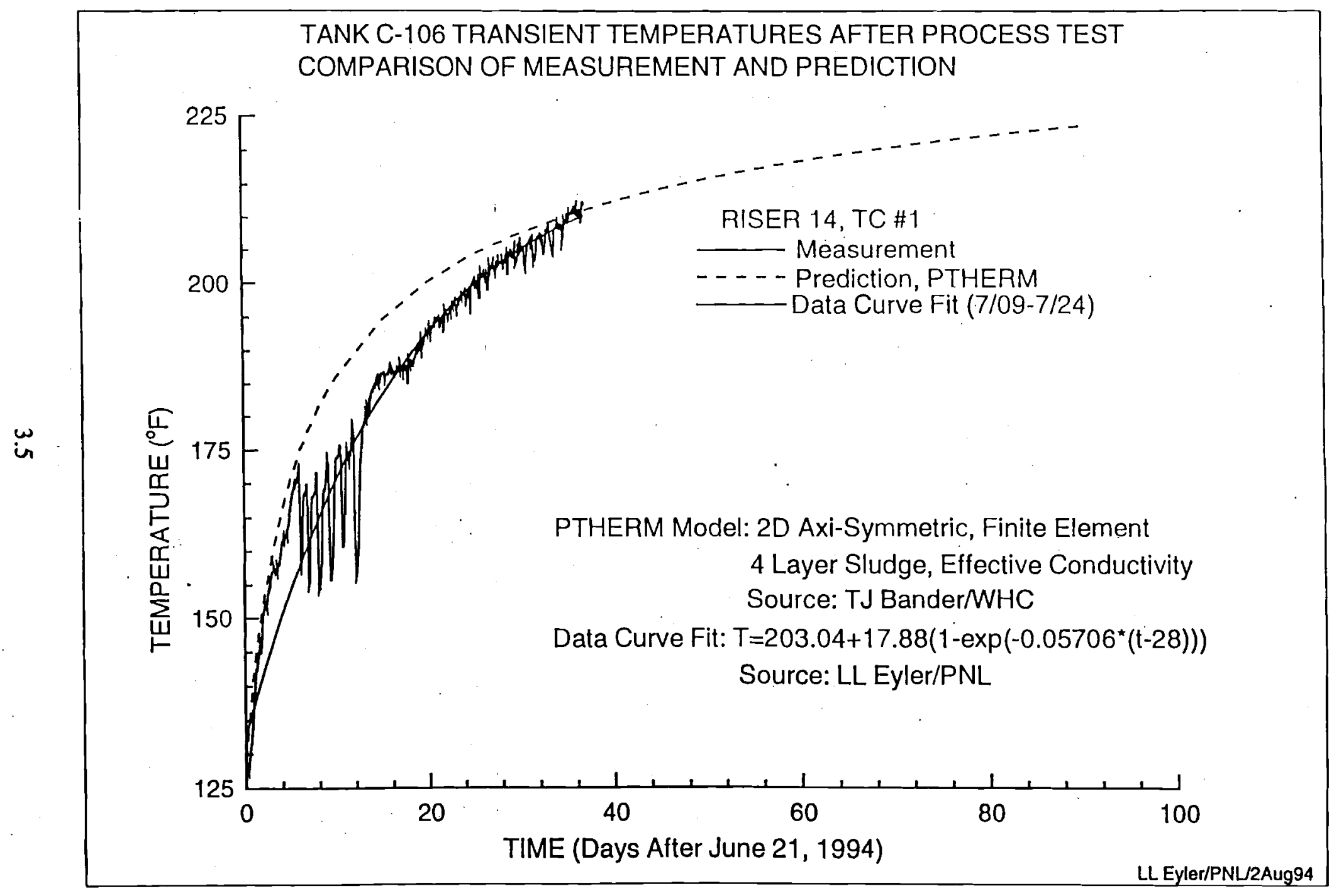

Figure 3.1. Comparison of Transient Temperatures in Riser 14 TC1 for Estimating Heat Up Time Response 


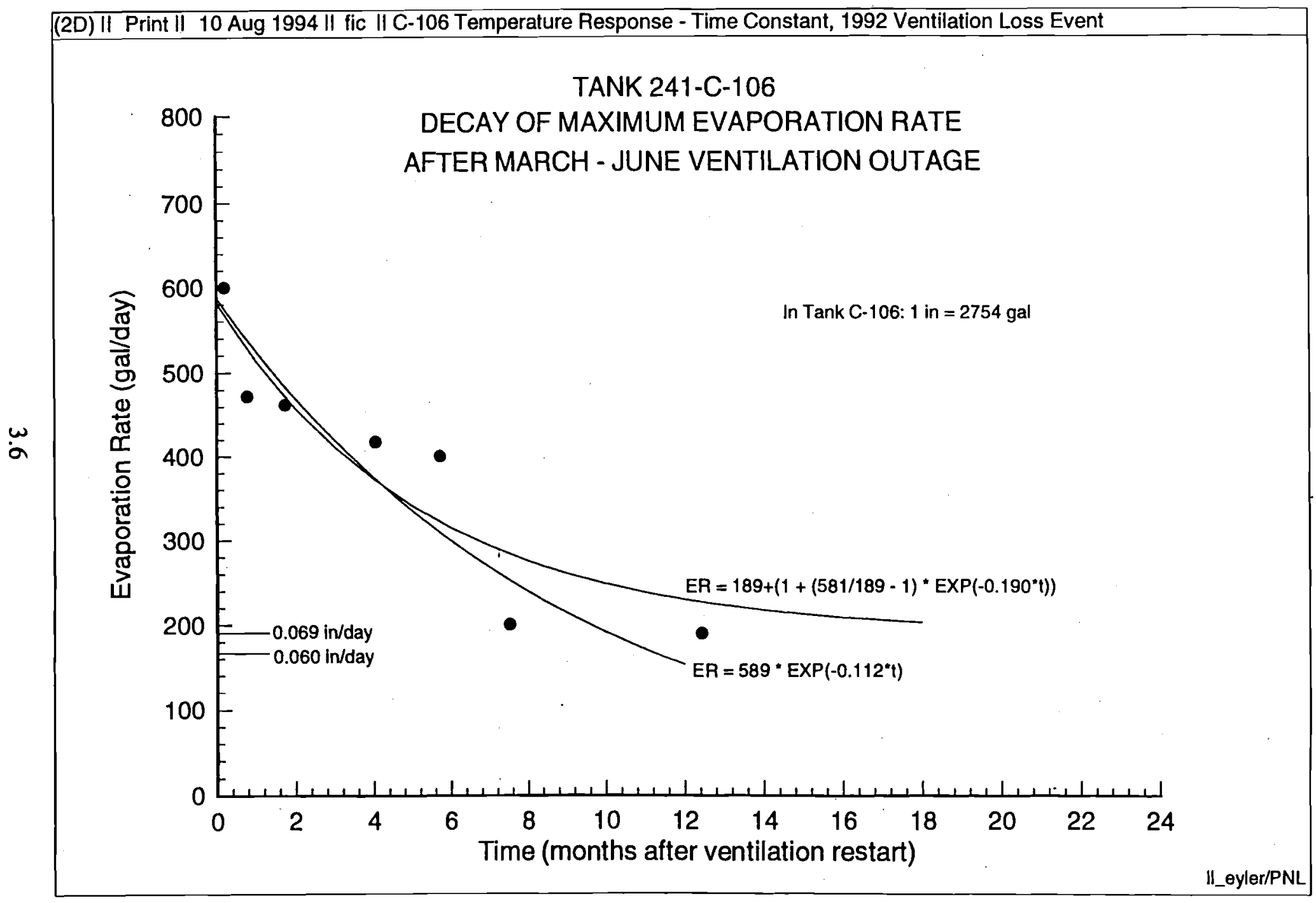

Figure 3.2. Decay of Maximum Evaporation Rate Following 1992 Ventilation Outage for Estimating Time Response 


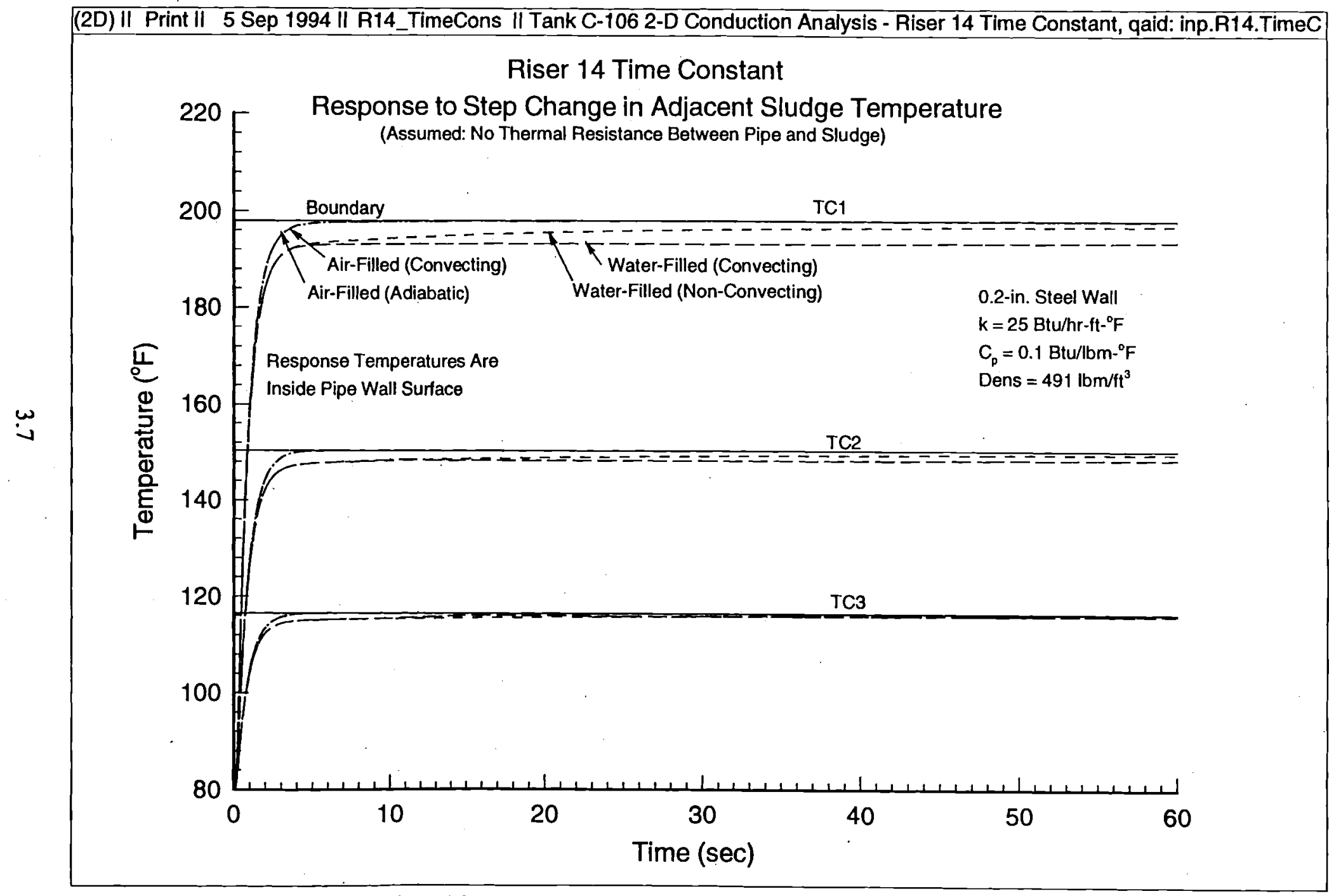

Figure 3.3. Response of Riser 14 to Step Change in Adjacent Sludge Temperature for Estimating Time Response 



\subsection{Riser 14 Temperature Indications Analysis}

As part of developing an explanation for the oscillations and other trends exhibited or interpreted from riser 14 temperature indications, several computer analyses were conducted on a model of the thermocouple tree in riser 14. The TEMPEST computer code was used for these calculations. The calculations were conducted with 2D and 3D cylindrical models of a region extending outward 6 and 15 feet from the centerline of riser 14. Included in these analyses were simulations that assumed sludge was in direct contact with the thermocouple tree and a convecting well was present around the tree. Both transient and steady state simulations of these configurations were conducted.

The objective of these calculations was to examine temperature fields around riser 14 subject to hypotheses about the near field adjacent to the tree. The aim was to assist in developing a defensible explanation for indicated temperature oscillations.

Several steps were taken in performing this analysis. A comparison of other computer modeling results are presented to set the basis. Calculations were conducted to examine temperature indication errors due to the presence of the thermocouple tree. Simulations, both steady state and transient, that examined the presence of a convecting well around riser 14 were conducted for tank conditions in about mid-May. Each of these analyses is discussed in the following sections. A discussion of the pertinence of the results to examining temperature oscillations is presented subsequently.

\subsection{Other Steady State Tank Modeling Results}

Computer modeling results of tank C-106 have been conducted by Bander (1993), Bander and Crea (1994), and Reid ${ }^{(a)}$. These analyses have been conducted using conduction modeling and assumed effective thermal conductivities. A comparison of computed results with riser 14 temperatures as reported by Bander ${ }^{(b)}$ is presented in Figure 4.1. These temperatures are typical for August 2, 1994. The agreement between Bander's and Reid's results is encouraging, but it must be noted that both analyses were conducted using similar finite element techniques. Therefore, the results are expected to be similar. They are not exactly the same because of differences in assumed effective thermal conductivities and heat generation distributions. In the lower region, the results are in closer agreement than in the upper region. Near the upper boundary, Bander's and Reid's results differ in the accounting

(a) H. C. Reid, September 1994. "Tank C-106 Assessment of Local Sludge Dryout." ASD/HCR94-002, Rev. 1. Pacific Northwest Laboratory, Richland, Washington. (Internal Letter Report to Westinghouse Hanford Company.)

(b) T. J. Bander. 1994. Personal communication. Results presented in Figure 4.1 constitute the continuous distribution temperature profile for the case reported by Bander and Crea (1994). 
for heat transfer across the liquid layer. In either case, it is not possible to conclude which results are in best agreement with data.

Also presented in Figure 4.1 is the temperature profile computed at the center of the tank (without consideration for the steam-jet structure known to exist there). A comparison is made to the saturation temperature distribution with depth as computed by Reynolds ${ }^{(a)}$. At the center of the tank, the computer model results indicate the temperatures exceed the saturation temperature. This result, in connection with two-phase computations conducted by Thurgood et al. (1994), is the basis for the conclusion that some boiling region is likely to exist in the lower, central region of the tank. The conclusion that a boiling region exists somewhere in the tank is key to explaining temperature oscillations indicated by riser 14 thermocouples.

One of the most significant uncertainties in the indicated sludge temperature is the exact location of the thermocouple junctions. This uncertainty is indicated by results presented in Figure 4.1. In this figure, continuous temperature distributions near riser 14 location and measured temperatures of August 2, 1994, are presented. The data are indicated by solid triangles. For each of the four measurement locations, TC1 to TC4, three locations are presented. The first location (lower triangle) is based on the thermocouple tree being 9.5 inches above the floor; the thermocouple locations are referenced from that position. This might be the case if the bottom conical section were a solid conductor of enhanced conductivity material. The second location (middle triangle) is referenced to thermocouple locations indicated on blueprint drawings. The third location (top triangle) is referenced to a verbal communication ${ }^{(\mathbf{b})}$ indicating that when this tree was installed, it was sluiced in, and may not have been completely inserted because of encountering the hardpan. Nearly nine inches difference exists between these three locations. Near the floor where the temperature gradient is very flat, this position variation is not remarkable. However, near the surface, the position difference is notable because it indicates the TC4 may or may not be below the liquid level, thus indicating supernatant temperature.

\subsection{Thermocouple Tree Temperature Error Estimation}

As a preliminary investigation, an analysis was made to estimate the local measurement error of axial heat conduction on thermocouples in trees in risers 8 and 14. Tank C-106 contains two thermocouple trees. The tree in riser 8 consists of a 3 -inch schedule 40 , seamless, ASME 304L low carbon stainless steel tube. Thermocouple junctions are located adjacent to the inside surface of the tube. The thermocouples are spaced 24-inch apart. Contact with the tube surface is enhanced by wadded aluminum foil packing local to each thermocouple.

(a) D. A. Reynolds memo to N. Kirch. August 3, 1994. "Estimated Boiling Point of the Liquid in 241-C-106." 7E310-94-039. Westinghouse Hanford Company (internal memo).

(b) O. S. Wang, Westinghouse Hanford Company, personal communication. July 1994. 
The bottom of the tree is flat and located 2.25-inch above the floor. A 1-inch diameter inner tube is in the center. This inner tube is open at the bottom, according to blueprints.

The tree in riser 14 is a 2-inch schedule 40, ASME 106 carbon steel, grade B. Thermocouple junctions in this tree are welded to plugs that are welded into the pipe wall. They are spaced at 24-inch intervals. The bottom of the tree is conical. The distance from the floor of the tank to the tip of the tree is 9.5 inches. The distance from the tip of the tree to the first thermocouple junction is 4 inches. This information is referenced by a memo from Irwin ${ }^{(a)}$.

To estimate temperature measurement error, a TEMPEST conduction computer model was developed. Figure 4.2 presents a schematic of the domain computed. It consists of 6-footradius cylindrical region around a tree. The outer boundary of this region is assumed adiabatic. For riser 8, a flat-bottomed, 3-inch diameter pipe with a 1 -inch inner cylinder was included. The outer wall thickness is 0.21 -inch. The distance off the floor was discretized at 2.4 inches. No credit is taken for the wadded aluminum packing. Thermal conductivity for 304L stainless steel is from Marks (1978):

$$
\mathrm{k}=8.08+0.0052 *(\mathrm{~T}-95)
$$

where $\mathrm{k}$ is in Btu/hr-ft-F and $\mathrm{T}$ is in degrees Fahrenheit. The gap between the interior and exterior pipes was assumed to be filled with air with $\mathrm{k}=0.016 \mathrm{Btu} / \mathrm{hr}-\mathrm{ft}-\mathrm{F}$. The inner pipe, open at the bottom, was assumed to contain non-convecting water with $\mathrm{k}=0.345 \mathrm{Btu} / \mathrm{hr}$ ft-F.

For riser 14, the tree was also assumed to be flat bottomed. The bottom of the tree was discretized at a distance of 9.6-inch above the floor. The wall thickness was modeled as 0.21 -inch thick. The tube was assumed to be filled with air. The thermal conductivity for the low carbon steel pipe is assumed to be $25 \mathrm{Btu} / \mathrm{hr}-\mathrm{ft}-\mathrm{F}$.

The computer solution used 30 radial and 60 axial nodes with the discretization concentrating cells in the vicinity of the thermocouple tree. Three or four cells were included in each pipe wall. The axial direction encompassed the distance from the tank floor, up through the sludge, the supernatant layer, and approximately 30 feet into the dome space. The top end of the tree was assumed to be at a constant temperature of $60^{\circ} \mathrm{F}$ (buried in concrete/dirt). The dome air temperature was assumed constant at $70^{\circ} \mathrm{F}$ for these simulations. The supernatant layer was assumed to be at a constant temperature of $85^{\circ} \mathrm{F}$. Steady state temperature calculations were done.

(a) Memo from J. J. Irwin to T. J. Bander, Westinghouse Hanford Company, "Location of TC Probes for Tank C-106," dated August 7, 1994. 
The sludge layer was modeled two ways. In one method, a linear temperature gradient varying from $215^{\circ} \mathrm{F}$ at the floor to $85^{\circ} \mathrm{F}$ at the sludge/supernatant interface was imposed at the outer domain boundary ( $\sim 6 \mathrm{feet}$ ). Using this method, variations in the linearity of the ambient temperature profile in the near vicinity of the thermocouple tree are indicative of the perturbing effect. The second method used a uniform heat generation rate in the sludge to approximate a parabolic temperature profile with a maximum value of $217^{\circ} \mathrm{F}$ adjacent to the tank floor. The profile approximates recently measured temperatures and other $2 \mathrm{D}$ conduction model analysis near riser 14 . Results of these two methods are largely the same and only the latter is presented here.

Figure 4.3 presents the results of the analysis for riser 8 . Included in the figure is a comparison of the temperature profile at a distance far removed from the tree ( $\sim 6 \mathrm{feet})$. It also presents the predicted temperature profile adjacent to the inner surface of the pipe wall. This location is assumed to approximate the location of the thermocouple junction. Also included in the figure is the difference between these two profiles. This difference is a measure of the local perturbation effect of the tree (the measurement error due to axial conduction). Note that throughout the greatest portion of the sludge layer, the error is less than $1^{\circ} \mathrm{F}$. The only significant differences occur in the vicinity of sludge/supernatant and supernatant/air interfaces. These differences occur because the thermocouple tree, being a better conductor of heat than the other materials, tends to smooth out the temperature gradient discontinuities. The largest variation occurs adjacent to the supernatant/air interface. The temperature difference in this location will vary regularly with ambient dome space temperature variation, supernatant temperature, and liquid level depth.

Figure 4.4 presents similar results for the thermocouple tree in riser 14. Differences between the thermocouple wall temperature and the ambient environment temperature are approximately $2^{\circ} \mathrm{F}$ over much of the tree's length in the sludge. This difference is greater than that in riser 8 because the thermal conductivity of carbon steel is nearly three times that of the 304L stainless steel. Again, the largest discrepancies are near interfaces. These differences will vary with ambient temperature changes in the dome space. Figure 4.5 presents results of a similar analysis that assumed the dome space ambient temperature was $100^{\circ} \mathrm{F}$.

For riser 8 and riser 14, the penetration depth of the supernatant/air interface temperature difference is less than one foot into the sludge depth. The nature and magnitude of local temperatures affected by the presence of the thermocouple tree in the sludge is consistent with conclusions reached in similar analyses conducted for waste tank $241-S Y-101^{(a)}$.

Numerous idiosyncracies of these thermocouple trees have been omitted or neglected in this analysis. For example, in riser 8 , the thermocouple junctions are welded interior to the pipe

(a) Z. I. Antoniak. "Tank 241-SY-101 Thermocouple Error Due to Axial Conduction Along Tree," memo to R. T. Allemann, dated December 29, 1992. 
wall and contact-enhanced by wadded aluminum foil. This local effect was not included in the modeling. Further, the axial conduction effect of thermocouple leads are neglected in this analysis also. Their effective cross-sectional area $(\sim 0.25$-inch diameter) is small relative to the cross-sectional area of the pipe walls. In riser 14 , the conical section on the bottom of the tree was approximated as a solid cylindrical section. It is not known what is interior to the conical section in the real tree. Its effect, however, is judged to be only a local one. Also in riser 14 analysis, the plugs the thermocouple junctions are welded to and then welded into the pipe wall were neglected. These plugs are small and judged to be an insignificant perturbation to the results.

No effort has been made in this analysis to investigate convective cooling effects of the interior of the thermocouple trees. Other analysis of thermocouple measurement errors exist $^{(\mathrm{a})}$ that examine other aspects. In reviewing that work, Bander and Crea (1994) concluded that thermocouple accuracy was $\pm 5^{\circ} \mathrm{F}$ and that repeatability was $\pm 4^{\circ} \mathrm{F}$.

The presence of the thermocouple trees on the measurement of local sludge temperatures is not a significant factor in explaining variations in temperatures recently observed in waste tank 241-C-106. This conclusion is based on analysis that assumes the sludge is directly in contact with the exterior pipe wall. Differences between ambient temperatures and those indicated by the thermocouple trees are less than $1^{\circ} \mathrm{F}$ for riser 8 and less than about $2^{\circ} \mathrm{F}$ for riser 14 over the largest portion of the sludge depth. Larger differences are predicted nearer the surface, but these are due to axial conduction in the thermocouple trees smearing the discontinuities in temperature gradients at the sludge/supernatant and supernatant/air interfaces. This latter interface temperature difference may be time dependent with daily dome space air temperature variations. The penetration depth in the thermocouple tree of this variation is estimated to be less than one foot below the sludge/liquid interface during a daily ambient temperature variation.

\subsection{Riser 14 TC4 Response to Water Additions}

Another indicator of response of the thermocouples in riser 14 is available by examining the correlation of temperature response of TC4 with water additions at the end of the process test from June 17 through July 3, 1994. A comparison between riser 14 TC4 temperature data and the approximate FIC surface level trend is made in Figure 4.6. For the first five water additions, TC4 reacted to the addition of water with a very direct and apparent response. The water added at this time was process water from the steam plant. While actual temperature measurements of the added water were not made, it was expected to be hot water because of its source and the length of time (usually overnight) it was allowed to stand in the water truck, prior to addition to the $\operatorname{tank}^{(\mathrm{b})}$.

\footnotetext{
(a) C. C. Scaief, June 1991. "Temperature Measurement Error Analysis." WHC-SD-WM-TI-483, Westinghouse Hanford Company, Richland, Washington.
}

(b) R. Ni/WHC, personal communication. 
Each of the first five water additions took approximately three hours to add approximately 2000 to 3000 gallons. A table of these additions is included in the Appendix. From the raw data used to plot the temperature data, the time to increase from the minimum temperature to the maximum temperature can be determined. It ranges from about 2.8 to 4.8 hours. These times are very consistent with water addition times.

The temperature increases that are measured by TC4 during the water additions are important to note. First, it can be concluded, from the correspondence of hot water addition and the indicated temperature spike, the thermocouple is responding and is indicating a measured temperature.

Based on analyses of TC locations from blueprints, TC4 should most likely be in a position corresponding to FIC surface level indicator of 77 inches. This position is based on a distance of 9.5 inches from the local floor to the end of the tree, 4.5 inches from the end of the tree to TC1, and 24 inches between each of the thermocouples. The local floor is 9.2 inches below the FIC zero reference at riser 14. Thus, TC4 should be at about 76.8 inches relative to the FIC indication. This position is above the liquid level for all but the last water addition after the end of the process test. No corresponding temperature spikes are identifiable in TC3 (24 inches below TC4). Thus, it cannot be concluded from riser 14 data alone that some effect above the liquid level (such as splashing) is not causing the temperature spikes at the water addition times. Thus, the most likely explanation for the temperature spikes is an axial conduction effect which was shown above to be very effective at smearing temperature differences across interfaces (see Figure 4.4, for example).

The historical data from the SACS database for risers 8 and 14, TC3, TC4, and TC5 are compared in Figure 4.7. Historically, TC4 on riser 14 is more closely associated with the temperature at TC3 (see also Figures 2.4 to 2.6). This observation seems to indicate that up until the time of the process test, TC4 and TC 3 were in good convective communication with the surface liquid. TC4 was then exposed to the dome space air when the liquid level dropped below 77 inches during the process test and it was covered again with the large 14,000 gallon water addition beginning on July 3, 1994.

TC4 temperature indications on risers 8 and 14 are plotted in Figure 4.8 with an apparent correlation between the two data sets. Riser 8 TC4 does indicate a temperature increase with the first five water additions in the same manner as riser $14 \mathrm{TC} 4$. The magnitude of the temperature increases is not quite as great. The most likely FIC surface level position of TC4 on riser 8 is 73 inches. Thus, it should also be above the liquid level for the first five water additions. This raises the question: if TC4 on risers 8 and 14 are above the liquid level for the first five water additions, and both are most likely submerged below the liquid level after the last large water addition on July 3, 1994, why are they not reading the same temperature for the liquid?

Several possible explanations for TC4 on the two risers to be reading different temperatures are presented. One possible cause is an axial conduction effect that is affecting the indicated 
temperature in each riser. A significant horizontal temperature variation may be present in the liquid. If this were the case, however, it might manifest itself over periods of time, but because water is added nearer to riser 8 , the temperature difference between riser $8 \mathrm{TC} 4$ and riser 14 TC4 should either diverge or coalesce, depending upon the relative temperature of water added and ambient supernatant temperature. This possibility is not observed in the data. The temperature indication of both TC4 thermocouples is that they are reasonably correlated. Prior to the last water addition of 14,000 gallons on July 3, 1994, the temperature difference appears to be a consistent $12-14^{\circ} \mathrm{F}$. After July 3, 1994 (day 55 in Figure 4.9), the difference between these two thermocouples decreases to a seemingly consistent 5 to $7^{\circ} \mathrm{F}$ until day 90 when riser $14 \mathrm{TC} 4$ indicates a $5^{\circ} \mathrm{F}$ drop. If the temperature difference horizontally was in fact present, it would have some effect on total evaporation rates and would be indicative of incomplete thermal mixing in the liquid layer.

Another possible explanation for the horizontal temperature variation exists. Videos taken in the tank 'in April and June indicated that a greater depth of sludge existed on the north side of the tank, where riser 8 is located, than nearer where riser 14 is located. These locations would have the effect of placing TC4 on riser 8 in a region more closely affected by sludge temperature due to axial conduction than TC4 on riser 14. Furthermore, the riser 14 thermocouple tree configuration is different than that of riser 8 . Riser 14 is a single 2-inch diameter carbon steel pipe; riser 8 is a 3-inch diameter stainless steel pipe that has an internal concentric 1-inch diameter pipe. According to drawings, the 1-inch diameter inner pipe is open at the bottom of the tree. This opening could have allowed the center pipe to be filled with liquid or sludge. The presence of this liquid would have the effect of more effectively communicating the thermal character at a shallow depth in the sludge to the position of TC4. Thus, it can be argued that riser TC4 should read hotter than riser $8 \mathrm{TC} 4$, even if both trees were subjected to the same surface liquid-layer temperature. 
Riser 14 Location

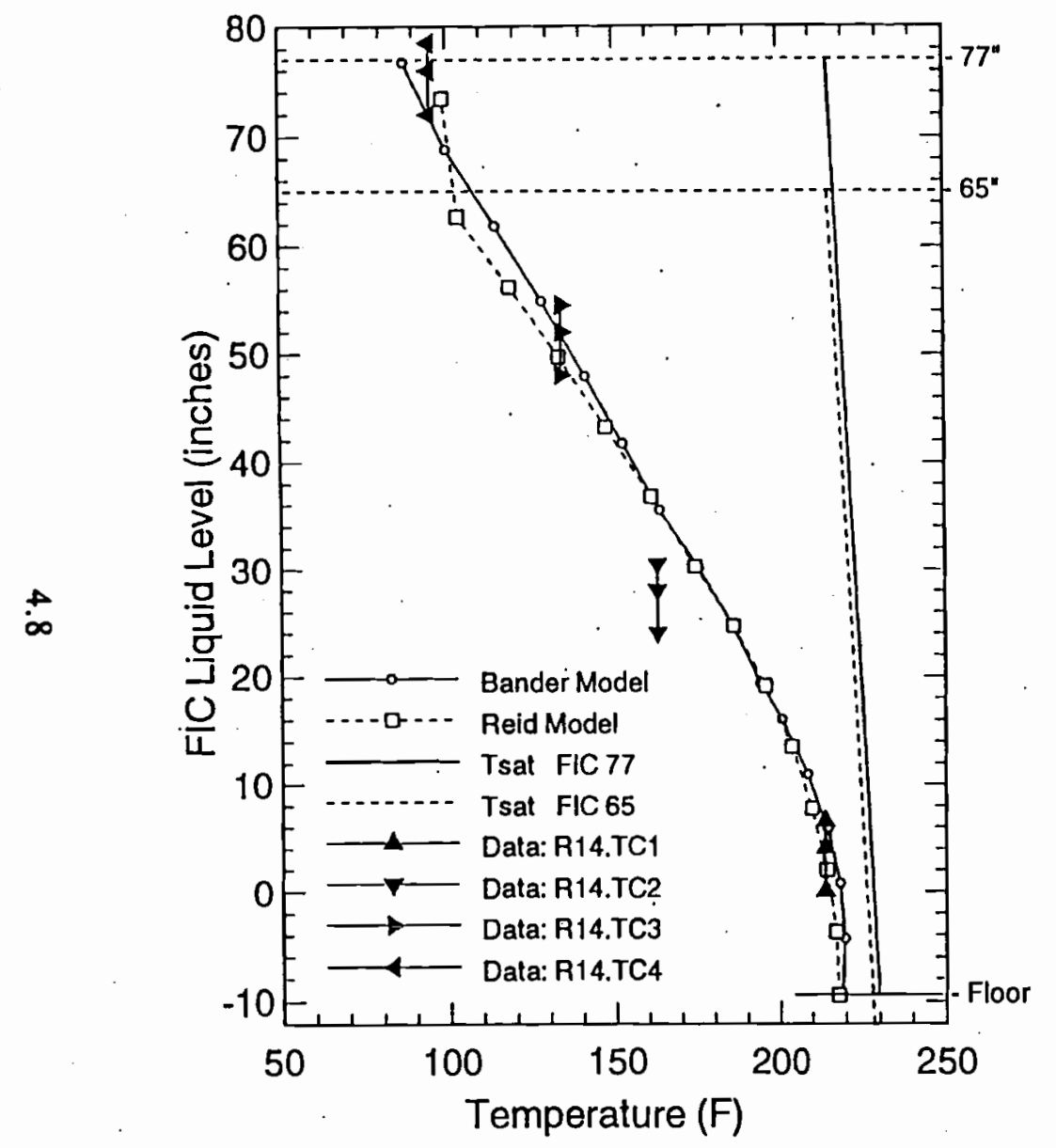

Tank Center

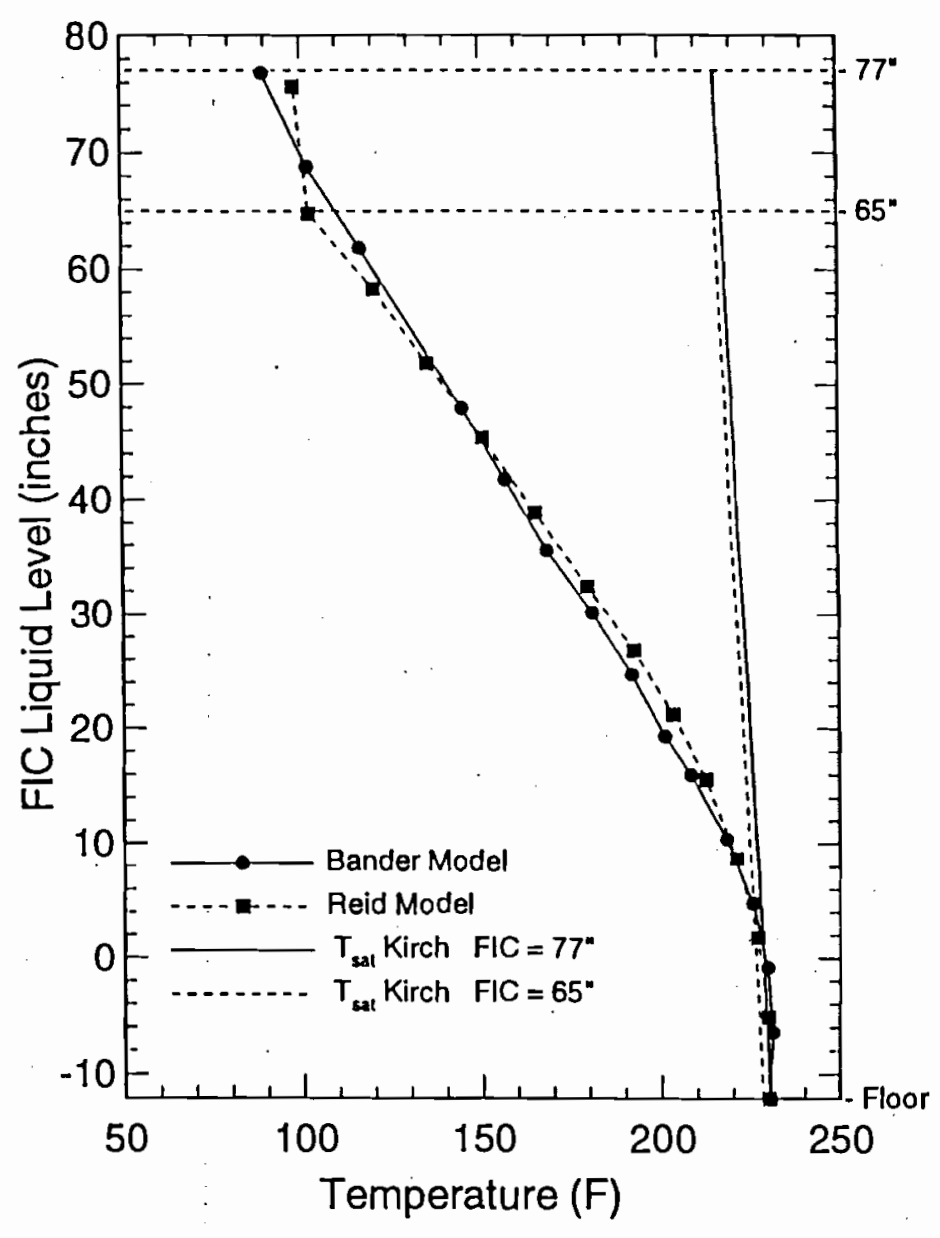

Figure 4.1. Riser 14 and Centerline Temperature Profiles 


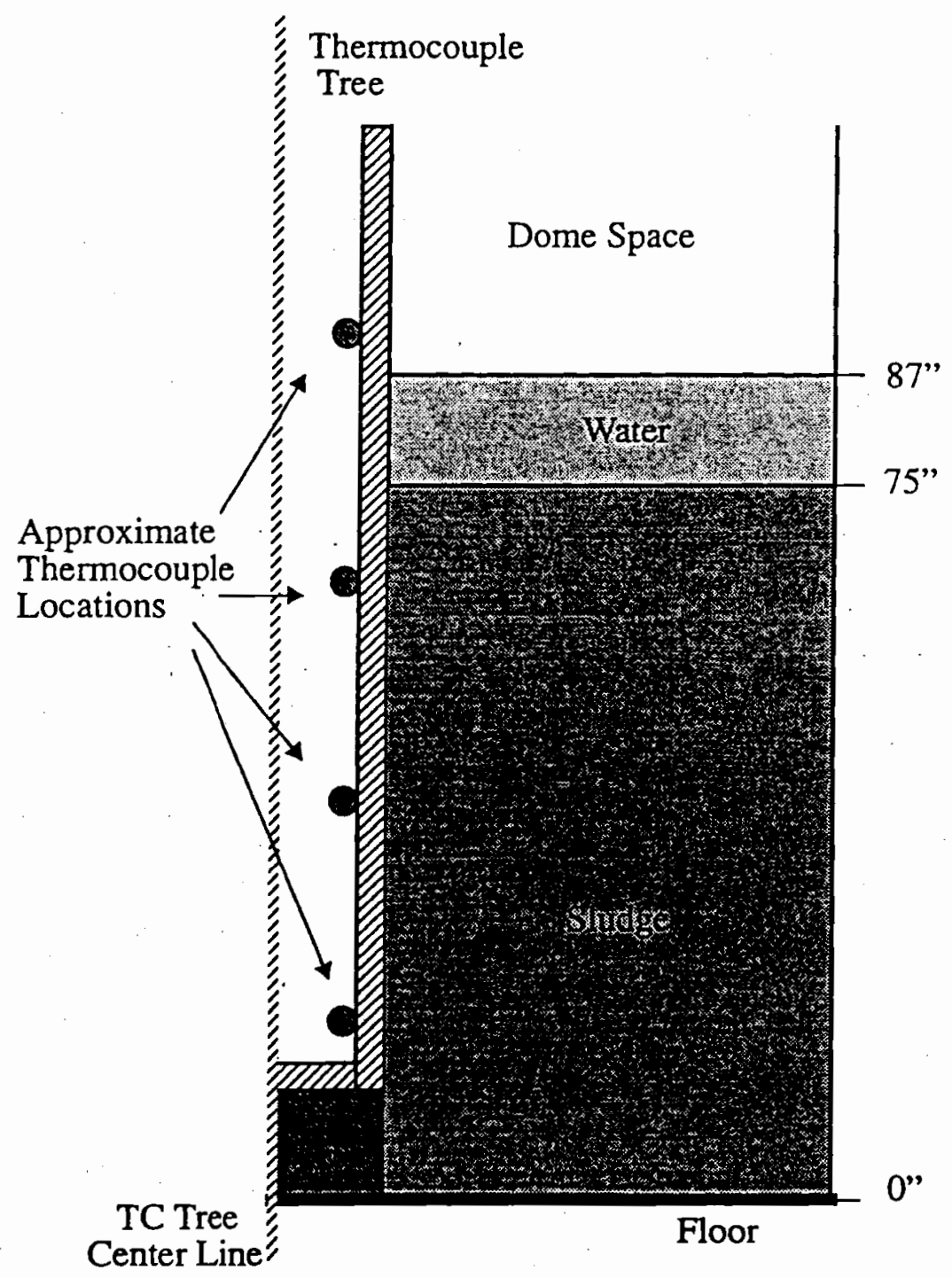

Figure 4.2. Schematic of Computational Domain for Investigation Thermocouple Response 


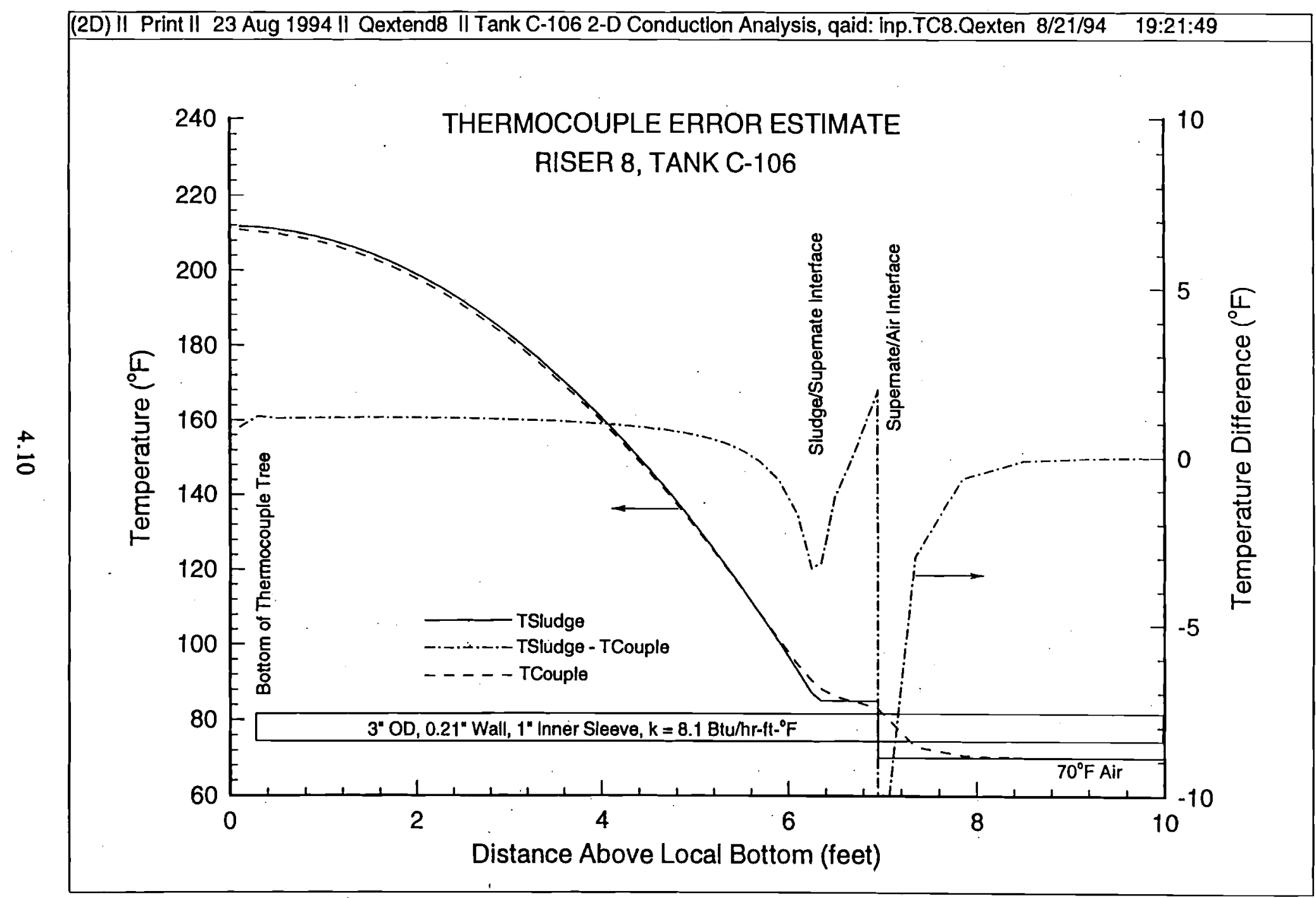

Figure 4.3. Riser 8 Thermocouple Tree Temperature Estimation Error for Axial Conduction 


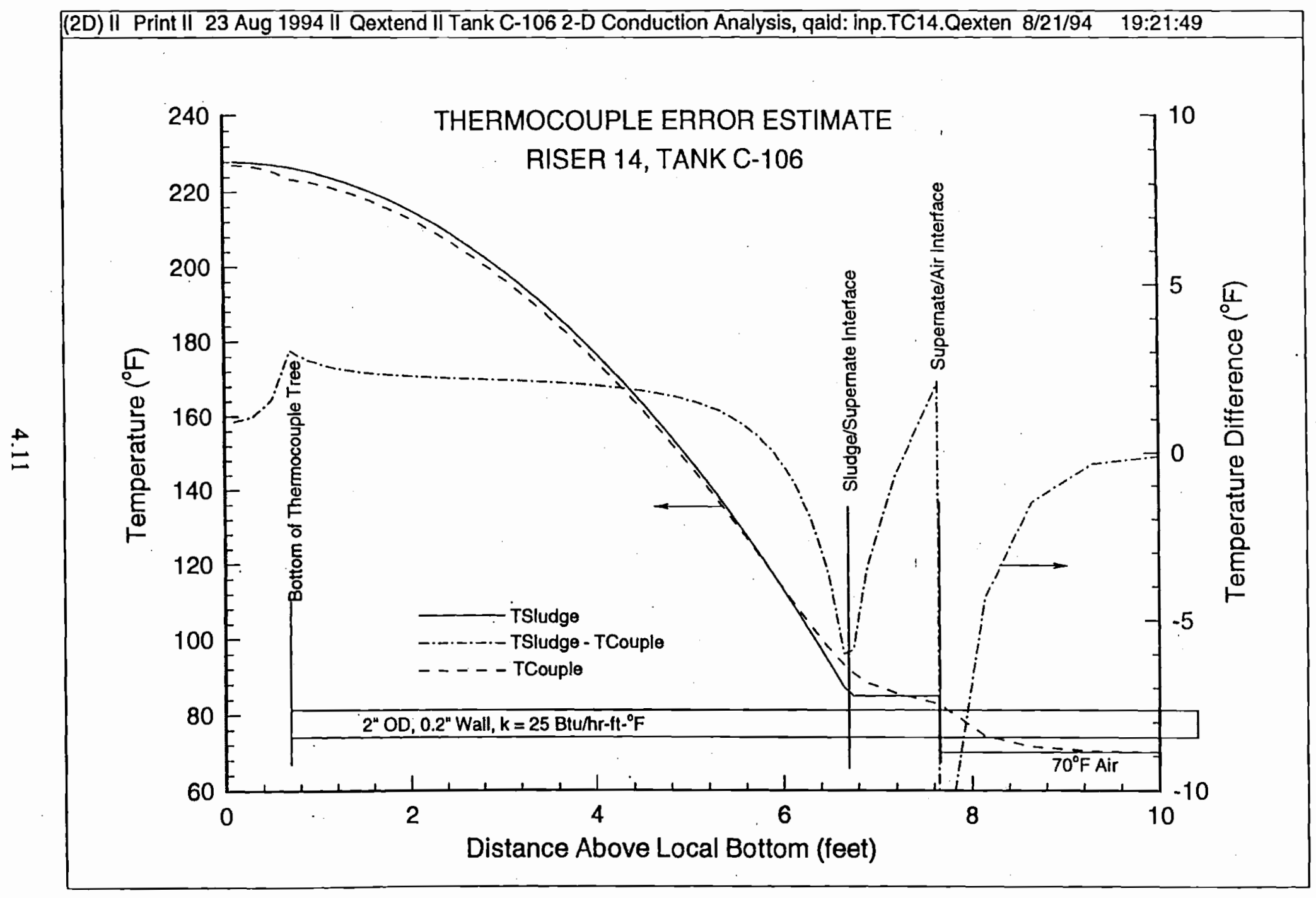

Figure 4.4. Riser 14 Thermocouple Tree Temperature Estimation Error for Axial Conduction with Colder Dome Space 


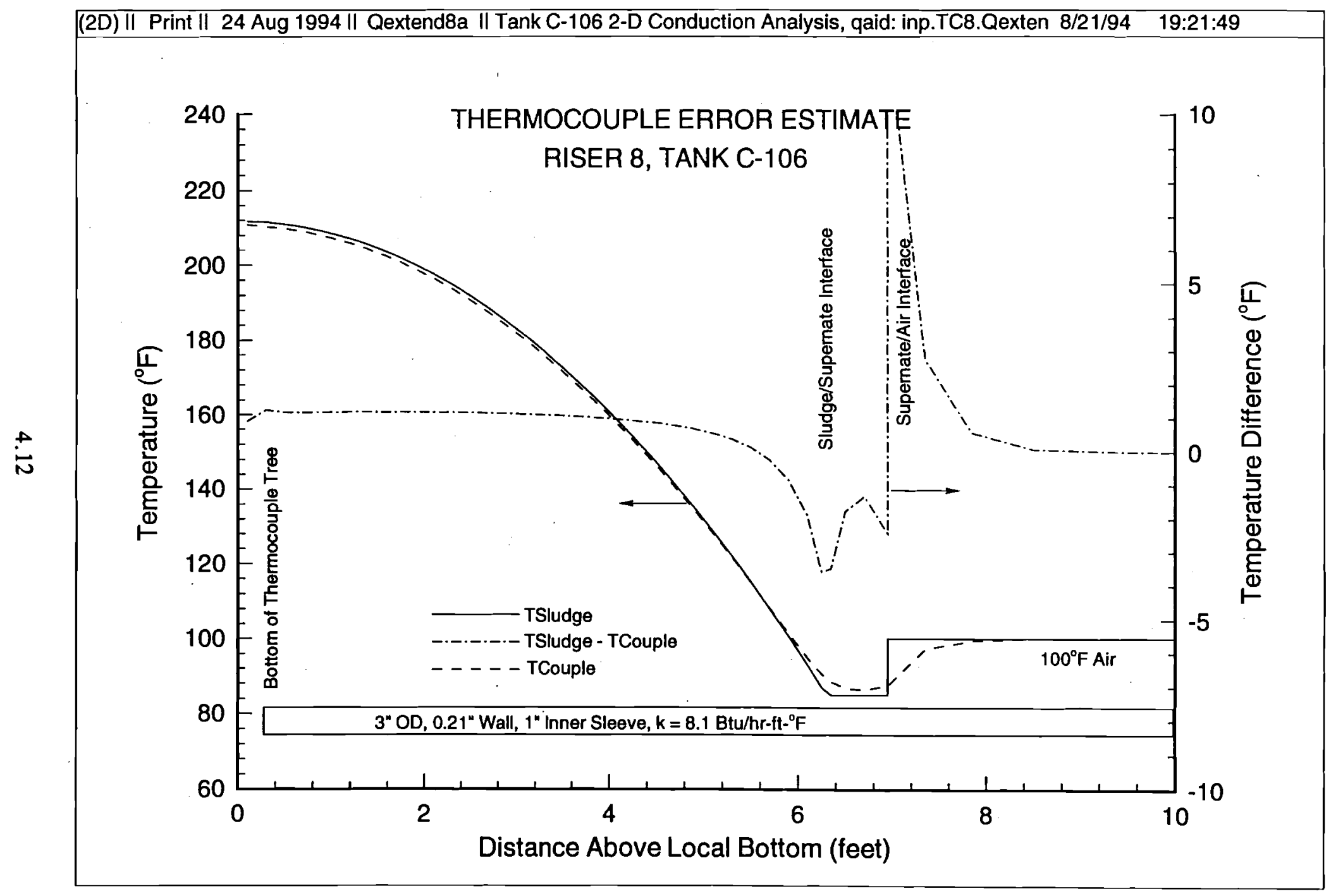

Figure 4.5. Riser 8 Thermocouple Tree Temperature Estimation Error for Axial Conduction with Hotter Dome Space 


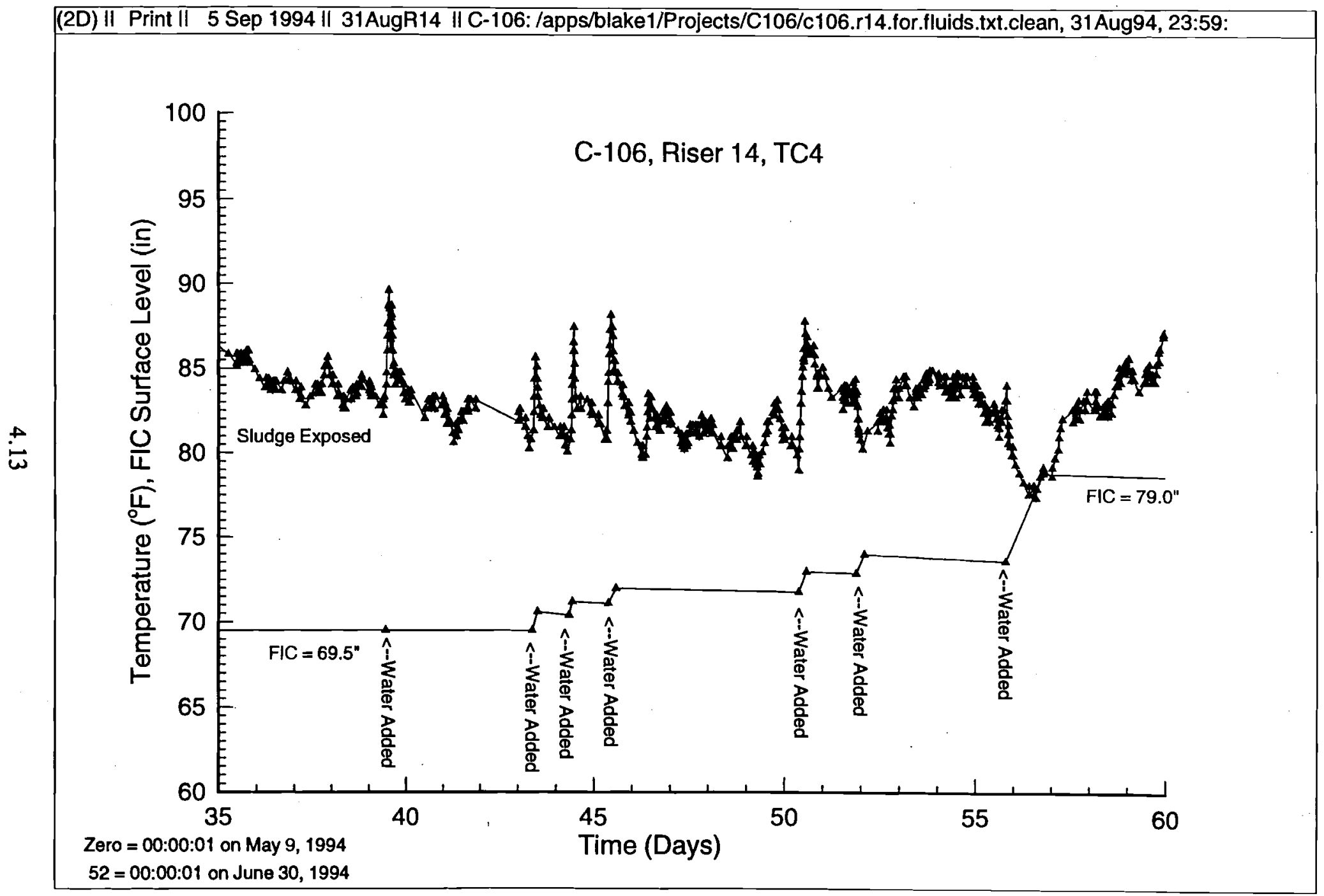

Figure 4.6. Response of Riser 14 TC4 to Water Additions after Process Test 


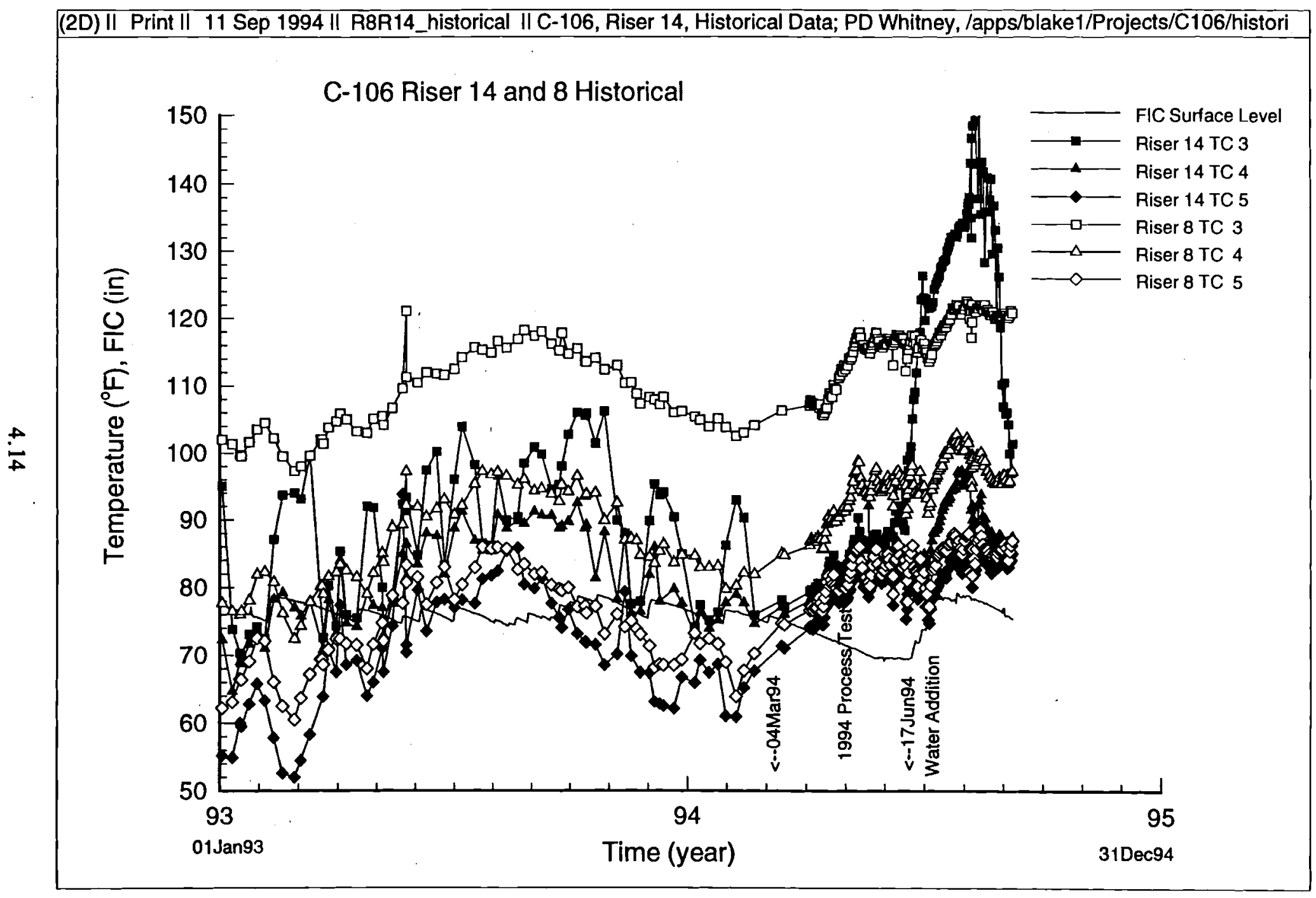

Figure 4.7. Comparison of Riser 14 and 8 TC3, TC4, and TC5 Historical Temperatures, 1993 and 1994, SACS Database 


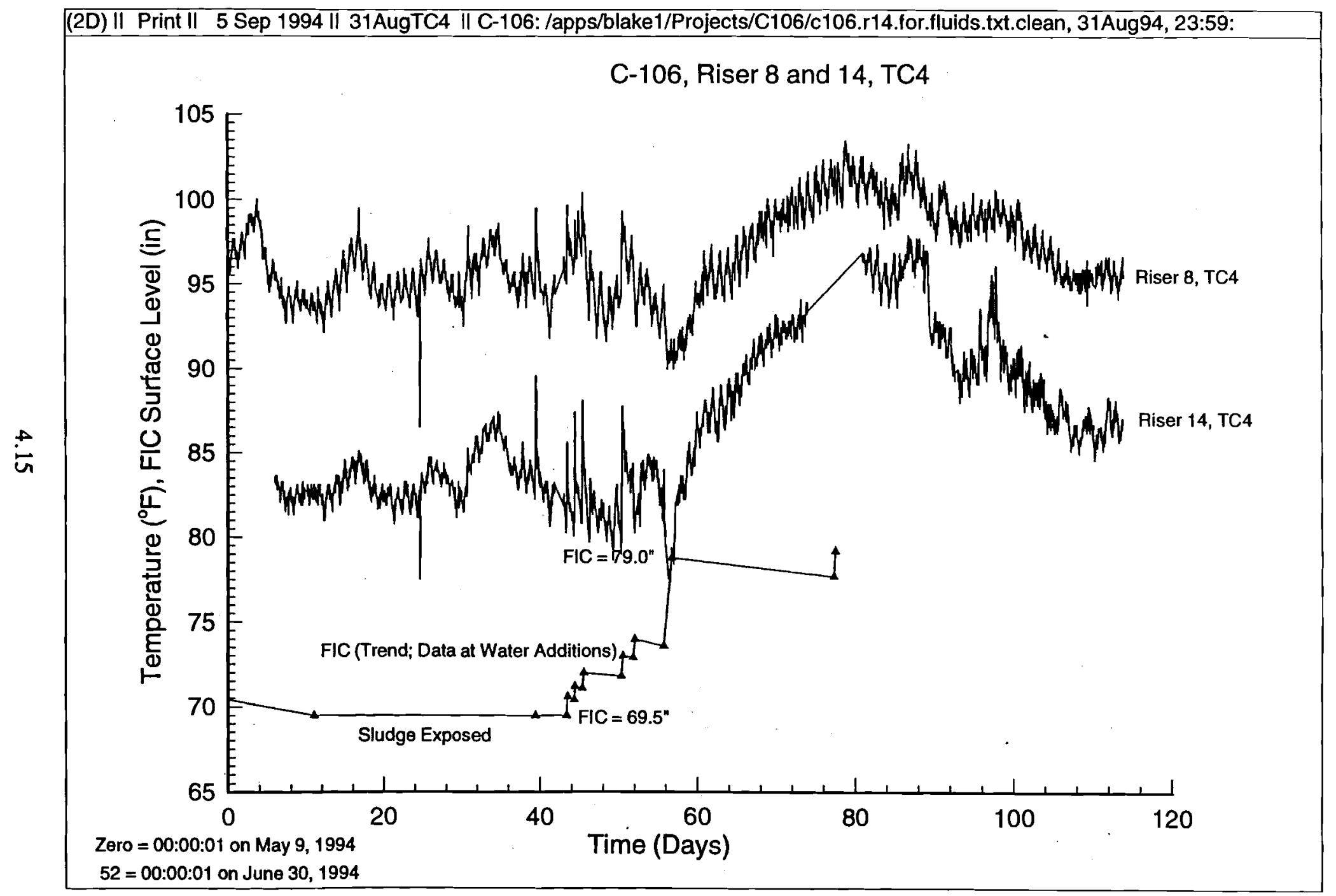

Figure 4.8. Comparison of Riser 14 and 8 TC4 Response to Water Additions - May to August 1994, TMACS Database 


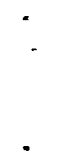




\subsection{Effect of an Assumed Well Around Thermocouple Tree In Riser 14}

Several simulations were conducted to examine the steady state and transient characteristics of the temperature field around the thermocouple tree in riser 14. The simulations were subject to assumptions regarding the presence of a well in the near field adjacent to the pipe wall. Results of this analysis are presented in several forms; quasi- and steady-state temperature distributions computed in the far field sludge and at the inner wall surface of the thermocouple tree pipe wall; transient temperature response to assumed convection in an adjacent well; and isotherms in the adjacent sludge field. The first of these results indicates that a local convecting-well-effect may have surrounded the thermocouple tree in riser 14 prior to and during early portions of the process test conducted between March and early June. The second of these results indicates the time response of such an assumed convecting well is shorter than observed in indicated temperature data. The third of these results indicate the presence of an assumed well does affect the temperature field surrounding the thermocouple tree so the magnitude of temperature oscillations (at least in some portions of the response transient) is of a consistent order.

For this analysis, the sludge was assumed to be layered in both thermal properties and in heat generation distribution. Following are the layer parameters.

\begin{tabular}{|c|c|c|c|c|}
\hline Parameter & Layer 1 & Layer 2 & Layer 3 & Layer 4 \\
\hline $\mathrm{H}$ & -9.5 to 0.0 & 0.0 to 15.5 & 15.5 to 39.5 & 39.5 to 69.5 \\
\hline $\mathrm{k}$ & 0.30 & 0.30 & 0.85 & 1.05 \\
\hline$\rho$ & 87.0 & 87.0 & 87.0 & 87.0 \\
\hline $\mathrm{C}_{\mathrm{p}}$ & 0.994 & 0.994 & 0.994 & 0.994 \\
\hline$Q$ & 5.4 & 6.4 & 3.5 & 1.0 \\
\hline
\end{tabular}

$$
\text { where } \quad \begin{aligned}
& \mathrm{k}=\text { Thermal conductivity }(\mathrm{Btu} / \mathrm{hr}-\mathrm{ft}-\mathrm{F}) \\
& \rho
\end{aligned}
$$

These parameters were selected during an iterative process attempting to improve the agreement of the conduction model with riser 14 data.

\subsection{Steady State Temperature Distribution - Sludge in Contact with Pipe Wall}

The steady state temperature distribution results for the case of the sludge in adjacent contact with the outer pipe wall are presented in Figure 5.1. Several sets of results are included in this figure. The solid triangles represent the temperature indications of TC1 to TC4 (bottom to top) in riser 14. These are data representing August 2, 1994, as presented by Bander 
(1994). Three points are shown for each thermocouple. The points represent approximate uncertainty in thermocouple locations in the tree, relative to the FIC surface level measurement. The solid circles are also data, but representing data in the mid-May time frame. In early August, supernatant temperature indicated by riser $14 \mathrm{TC} 4$ was about $95^{\circ} \mathrm{F}$.

The results of Bander and Reid's finite element conduction analyses are also included in the figure. They are the profiles marked by the open squares and circles, respectively. Also included for the August profile are the present model results which use the layers previously defined. It is difficult to conclude that one result is a closer match than the other. The earlier analysis better matches data at TC1 and TC3, whereas the present model better matches data at TC1 and TC2. For the present results, a temperature profile in the sludge (far removed from the thermocouple tree) is included as the solid line and a temperature profile at the interior pipe wall is included as a dotted line. As with the previously discussed thermocouple temperature error due to axial conduction in the pipe wall, the largest deviations are in regions with significant changes in thermal conductivity. This situation occurs in the lowest region, near the lower end of the thermocouple tree in the present layered model.

Also included in Figure 5.1 are the computed results for the mid-May case where the temperature of the supernatant is assumed to be $80^{\circ} \mathrm{F}$. For this case, the conduction model analysis that used the same parameters as the August case is in much greater disagreement with the indicated data. This observation is not earth shattering, but simply restates previous conclusions that some local perturbation must exist around riser 14 that causes it to indicate much lower temperatures than would logically be expected at this location. Before discussing this anomaly further, it is worthwhile to mention a very important factor, the magnitude of thermal conductivity.

For either the present result or that of Bander or Reid to even reasonably approximate the indicated temperature data of August 2,1994, it is necessary to use effective thermal conductivity values in the upper two thirds of the sludge that are in excess of the thermal conductivity of water $(0.36 \mathrm{Btu} / \mathrm{hr}-\mathrm{ft}-\mathrm{F})$. Without measured thermal conductivity data for C-106 tank sludge, or a definitive analysis of a better way to estimate it, the best approach is that used in this and previous analyses: thermal conductivities are adjusted until an approximate agreement is found between predictions and indicated data. It should also be noted that no analysis has been conducted of tank C-106 sludge that assumes it behaves more like a porous media than an unconsolidated particle/liquid medium.

Another result in Figure 5.1 is in regard to the predicted temperature profile between midMay and August 2,1994. As the supernatant boundary condition temperature is lowered from 95 to $80^{\circ} \mathrm{F}$, the maximum temperature (and in fact, the whole profile) is lowered the same number of degrees. This is a direct consequence of the effective thermal conductivity conduction model approach. The steady state temperature profile, and hence heat transfer in the sludge, is linearly dependent upon the supernatant temperature. This occurs because the supernatant temperature is the ultimate heat sink for the sludge. The linear dependency is in 
effect as long as the heat transfer out of the bottom and the sides of the tank are a relatively small fraction of the total heat transfer to and through the supernatant layer. Although the supernatant temperature changes due to environment changes in the dome space, the sludge temperature will always lead or lag the equilibrium steady state value. The near-linear dependency of the maximum temperature in the tank with the supernatant temperature is a good argument for cooling down the sludge by cooling down the supernatant. In this case, the time lag in stored heat in the sludge must be considered also.

The importance of the preceding results and discussion is that, at present, the effective thermal conductivity modeling approach is the best approach demonstrated to approximately predict the indicated temperature distributions. For that reason, the approach is used further in the present analysis to investigate assumed local well effects around the thermocouple tree in riser 14.

\subsection{Steady State Temperature Profile in Riser 14 Due to an Assumed Well}

To compute the effect of an assumed well on the temperature profile in riser 14, assumptions must first be made as to the nature and extent of the well. Parameters that could affect the temperature indications are width, depth, boundary interface shape between the well material and the ambient sludge, communication with the surface liquid, heat generation, and flow capabilities of material in the well. The parameter space is very large, so several simplifying assumptions are made.

In the first analyses, it is assumed the well surrounding the thermocouple tree is of uniform thickness and cylindrically concentric around the pipe. Thicknesses of the well of 1,2,3, and 6 inches were computed. In one calculation, the well was approximated as a conical shape with a 1-inch gap at the bottom and a 6-inch gap at the top. Fluid in the well was first assumed to be water, as if the well had open convective communication with the surface liquid layer. The result of this analysis indicated almost immediately the temperature of the thermocouples in the well would equilibrate to the surface liquid temperature. This situation resulted because the rather low viscosity of water allowed significant convection in the well. This assumption would not show support for the axial temperature gradient that exists in all the indicated temperature data for riser 14.

Subsequently, it was assumed the well was filled with a viscous liquid-like material with a viscosity $10^{3}$ times that of water. For the mid-May conditions, steady state results for this case are presented in Figure 5.2. Included in the figure are the August results and the midMay results for sludge in direct contact with the outer pipe wall. These latter results are the basis for evaluating the cooling effect of the convecting well. Shown in the figure are the steady state temperature profiles at the inner pipe wall for the assumed well shapes and sizes. Note the 3- and 6-inch gaps, as well as the conical gap, equilibrate to very nearly the constant surface liquid temperature. 
The 2-inch gap is in approximate agreement with the measured data, but does not well describe the temperature profile. For the assumed fluid conditions, the 1-inch gap does not provide sufficient cooling. In these analyses, the foot of the thermocouple tree is embedded in the hardpan layer at the bottom of the tank. For this reason, locally higher temperatures are predicted just at the end of the thermocouple tree.

While not providing a definitive proof, these results do show that a viscous, convective layer surrounding the thermocouple pipe could provide the type of cooling necessary to replicate the indicated temperatures in riser 14 prior to and during the process test. This cooling effect has certain implications to the temperature transients observed since the conclusion of the process test. These implications are discussed subsequently.

\subsection{Quasi-Steady Response of Riser 14 Thermocouples to Convection in an Assumed Well}

The next step in the analysis is to compute the transient response of thermocouples in riser 14 due to convection in an assumed well. For this analysis, the same concentric annulus configurations are used as above. For initial conditions, the steady state temperature distribution for sludge adjacent to and in contact with the pipe wall was computed. This is shown as the curve labeled ambient sludge for the mid-May supernatant temperature shown in Figure 5.2. Convecting fluid with a viscosity $10^{3}$ times that of water was assumed to be initially at rest in the gap. A temperature transient of up to one hour was then computed for each gap width and configuration. The time-dependent transient temperatures are presented subsequently.

The temperature profile in the thermocouple tree after a period of one hour is presented in Figure 5.3. By the end of this time, the temperature response of the fluid in each gap configuration had reached nearly an equilibrium condition. That is, the fast response temperature change of the fluid in the gap had come to equilibrium. The slow response change for the fluid to completely effect cooling of the surrounding sludge is a much longer process. In the long term, the steady state temperature profiles in Figure 5.2 would be reached. For reference, the previously discussed steady state temperature distributions for the no-gap cases are included in the figures. Note, the temperature distribution computed for these cases is quite similar in character to the indicated temperature data. Even the 6-inch gap configuration is in approximately good agreement.

While not definitive, these quasi-steady temperature profile results and those of the steady state results in the previous section indicate the possibility exists of a well configuration with some sort of enhanced convective cooling effect that can explain the historically low riser 14 temperatures. These results also support the historical observation that riser 14 TC3 and TC4 are in agreement much of the time, particularly in the 1993 and 1994 pre-process time frame (see Figure 2.6, for example). 


\subsection{Transient Response of Riser 14 Thermocouples to Convection in an Assumed Well}

The transient response time of an assumed well is presented in Figures 5.4 through 5.6 for TC1, TC2, and TC3, respectively. In each figure, the thermocouple temperature response is plotted as a function of time for different assumed gaps. Of particular importance is the thermal response time of the convection process in each gap is relatively short, between 5 and 20 minutes. The transient characteristic after the initial response is when the convection temperature in the gap comes into equilibrium with the surrounding sludge. The heat capacity of the sludge is large, due to the volume of material. Therefore, the long-term response of the convecting well is controlled by the surrounding sludge temperature and heat capacity, not the other way around.

By way of comparison, Figure 5.7 presents a comparison of riser $14 \mathrm{TC} 1$ temperatures for several large, fast transients observed in the day-to-day temperature response. These have been expanded in a 1-day time plot. Time is shifted to show each transient response in a 24hour period from midnight to midnight. The oscillations plotted are one of the periodic oscillations from June 30, 1994 and two oscillations from the more chaotic period in early August. Also shown in the figure is one of the temperature transients of the gap response calculation.

It is readily apparent the time responses of these two systems are quite different. The conclusion is the thermal time constant of the assumed gap configuration surrounding riser 14 is not a limiting factor affecting the time response of the riser 14 thermocouples. Smaller gaps than those considered here would respond faster thermally and be even less restrictive.

\subsection{Steady State Sludge Temperature Field Surrounding Riser 14 with an Assumed Well}

The effect of the assumed well surrounding riser 14 in the long-term, steady state can be examined from the steady state thermal field in the sludge surrounding the riser 14 thermocouple tree. These fields were computed by solving the fully coupled convection process in the well with the sludge field thermal conduction using a steady state solver method in TEMPEST. The solution domain for these calculations spanned 15 feet from the riser. The steady state isotherms in sludge are presented in Figures 5.8 to 5.12. Several observations are obvious and significant in these results.

One obvious observation is that a well with a larger gap is more effective in cooling a larger volume of sludge. A less obvious, but significant observation, is the smaller the gap, the greater the propensity for multicellular convection to develop in the gap. Such multicellular convection has a different net cooling characteristic. The impact of these results is that if small sludge motions close the gap by lateral movement, the temperature of sludge that comes into contact with the tree, and hence the thermocouple temperature response may be 
different, depending upon the local temperature field in the sludge. The local steady state temperature field in the sludge is correspondingly coupled to the convection process in the gap.

\subsection{Transient Temperature Response of Sludge Due to Assumed Closure of Well Gap}

If a thin, convectively cooled annular gap region exists and small amounts of lateral sludge motion close that gap, the surrounding sludge that had been cooled by the fin-cooling effect of the thermocouple tree and convection in the gap should exhibit a transient conduction heat up. This process was examined by computing a transient conduction process that approximates the significant temperature increase observed in riser $14 \mathrm{TC} 1, \mathrm{TC} 2$, and TC3 beginning approximately June 21,1994 (day 44 in Figure 5.13). This calculation is similar to the one on transient temperatures done by Bander (1993) (see Figure 4.1). Initial conditions for this calculation were the steady state thermal field surrounding the thermocouple tree.

Figure 5.13 presents a comparison of the indicated temperature data and the predictions of the conduction heat up. For this calculation, two sets of results are presented, one that maintains the liquid temperature at $80^{\circ} \mathrm{F}$ over the whole transient range and one that maintains a liquid temperature more closely tied to TC4 in riser 14 . The liquid temperature is used as an upper boundary condition to this calculation. The objective of this calculation is to determine if the initial portion of the indicated temperature increase can be described by a conduction heat-up process that begins from a temperature field produced by riser 14 being in an assumed well. In comparing the transient results, it is apparent the initial portion of the heat up is well described by the conduction process. This conclusion is consistent with ones drawn by Bander.

At least two other observations about this comparison are noteworthy. A defined deviation appears to be present in the predicted results for the two surface temperature conditions. Such a deviation also appears to be evident in the indicated temperatures by a change in slope. These two trends are marked by the lines in Figure 5-13 labeled Slope Change, Data and Model. The slope of the line marked Data through the indicated temperatures should be a measure of the time necessary for a liquid surface temperature perturbation to be sensed in the sludge. The time indicated by the data is about 6 to 8 days. This information may be very useful in determining analysis of alternate cooling methods applied to this tank. It would be a measure of the time it would take for a surface liquid temperature change, due to an alternate cooling method, to be effective at the bottom of the sludge. 


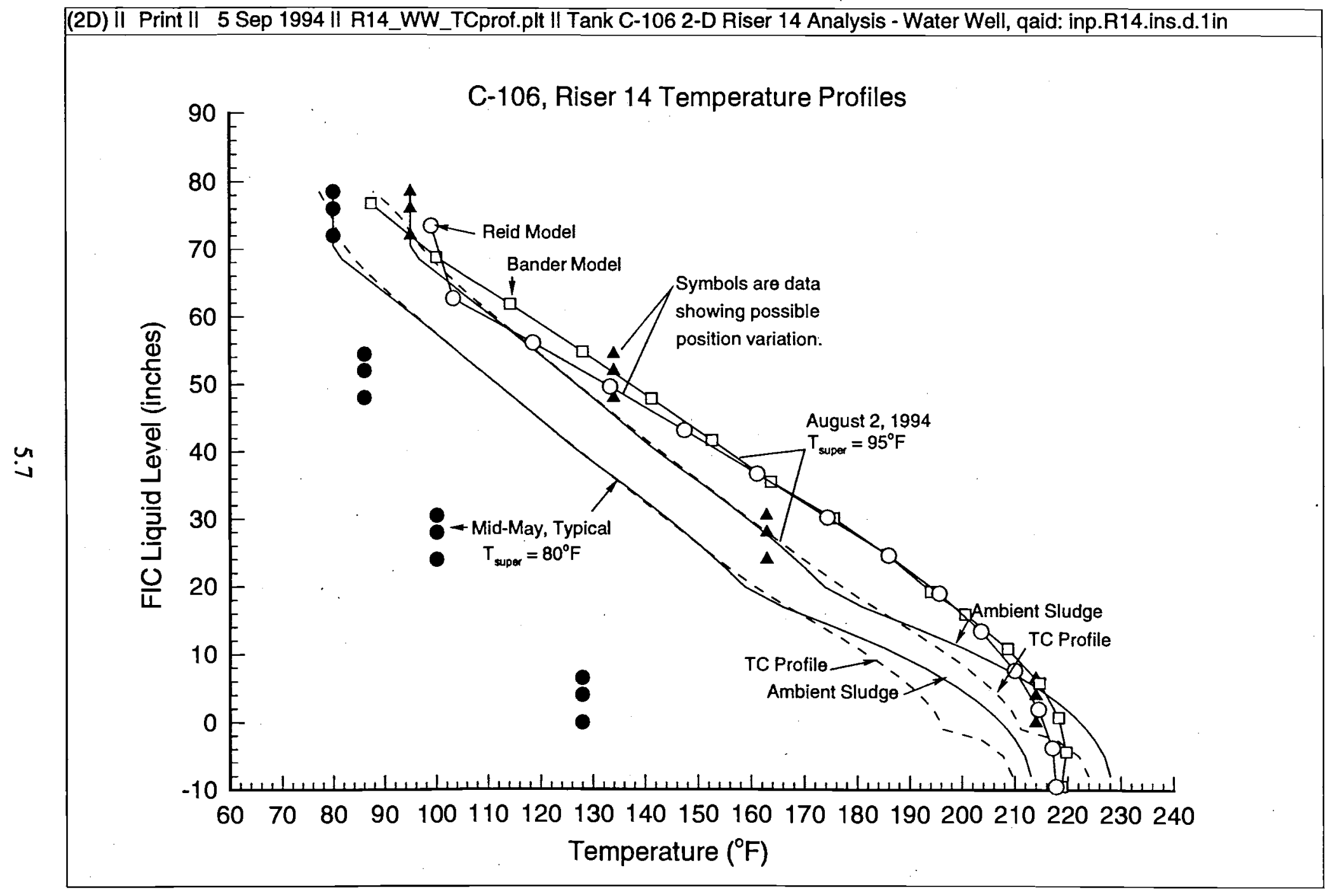

Figure 5.1. Comparison of Riser 14 Predicted Steady State Temperature Distributions for Two Supernatant Temperatures 


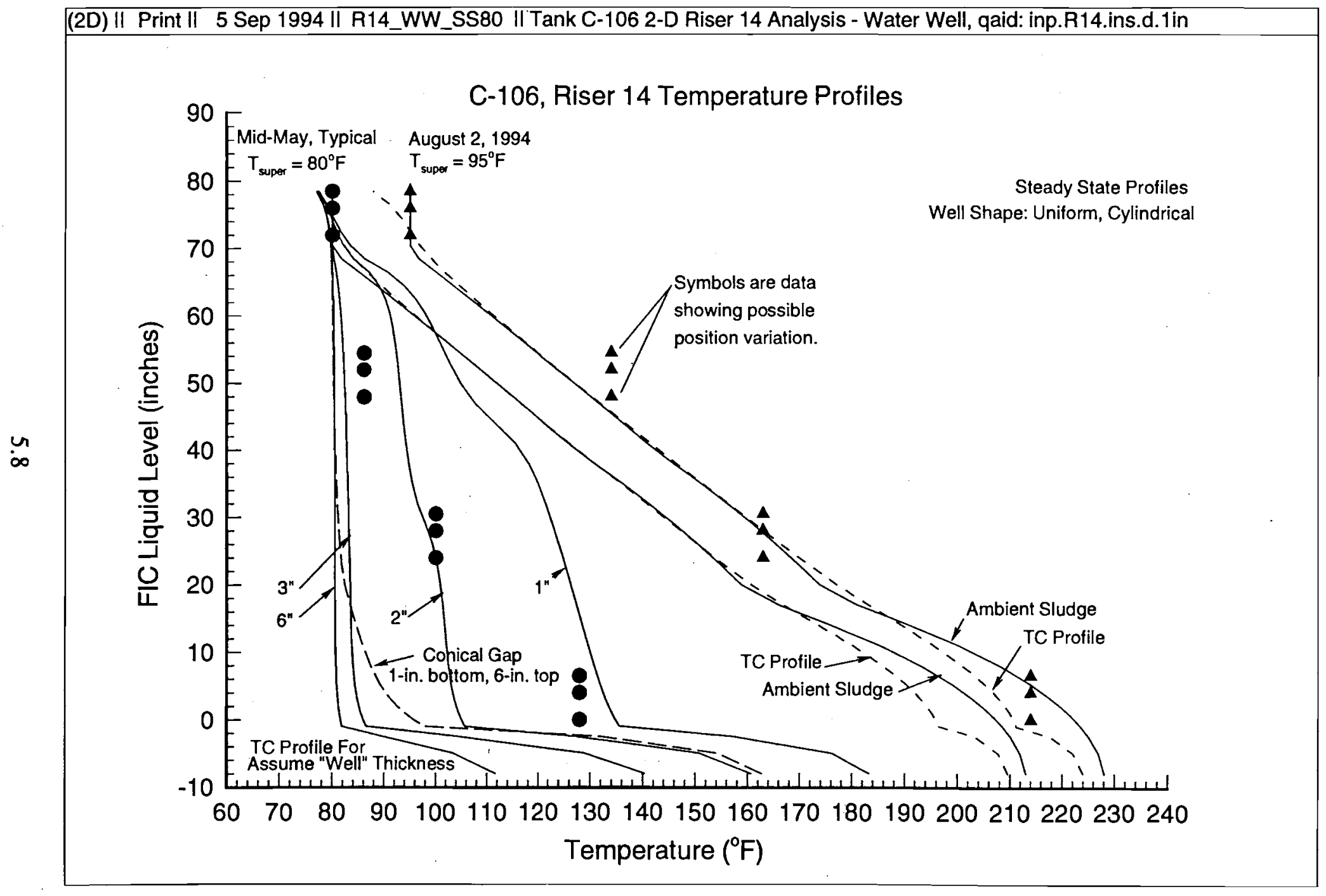

Figure 5.2. Comparison of Riser 14 Steady State Temperature Profiles for Assumed Gap Widths 


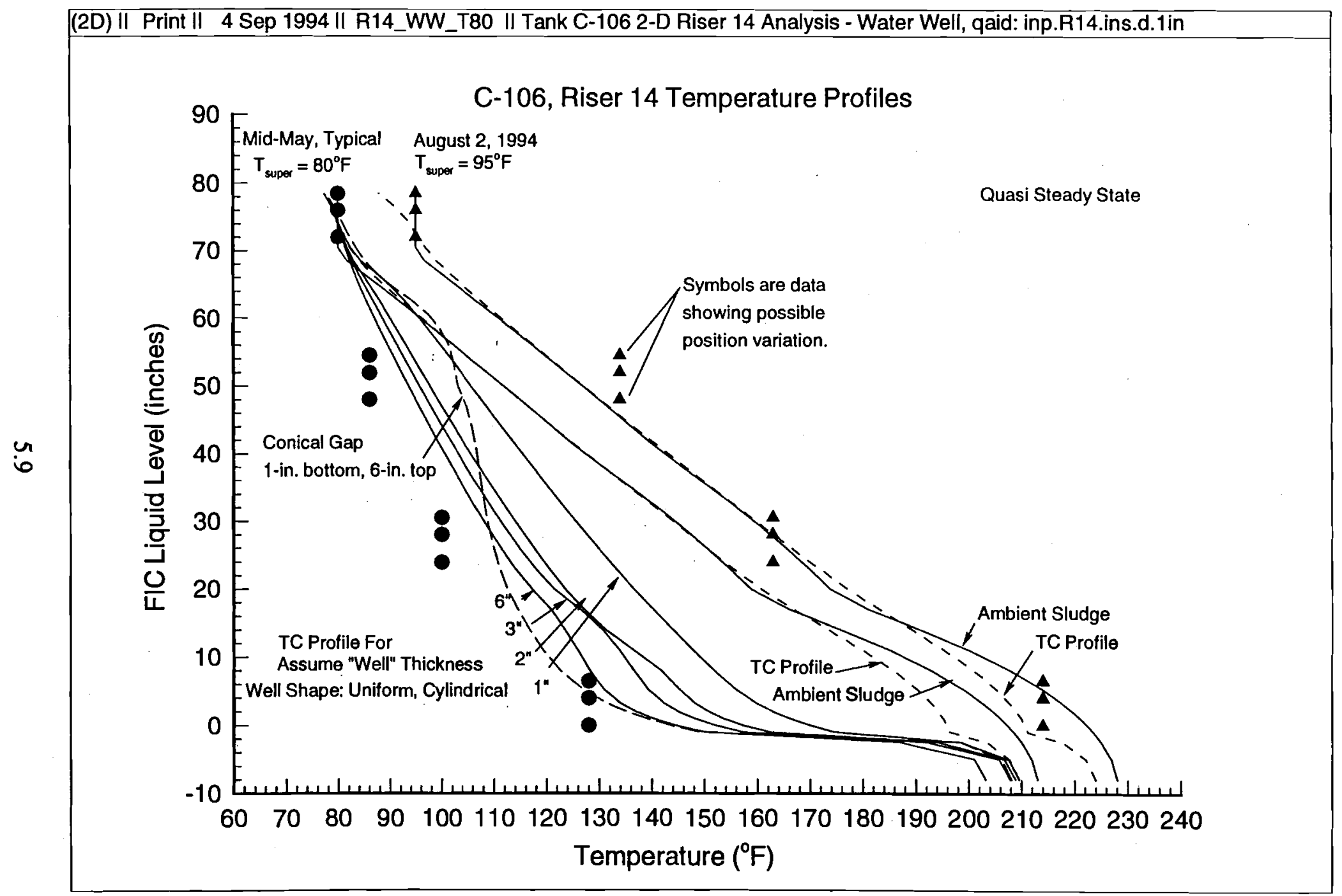

Figure 5.3. Comparison of Riser 14 Quasi-Steady Temperature Response for Assumed Gap Widths 


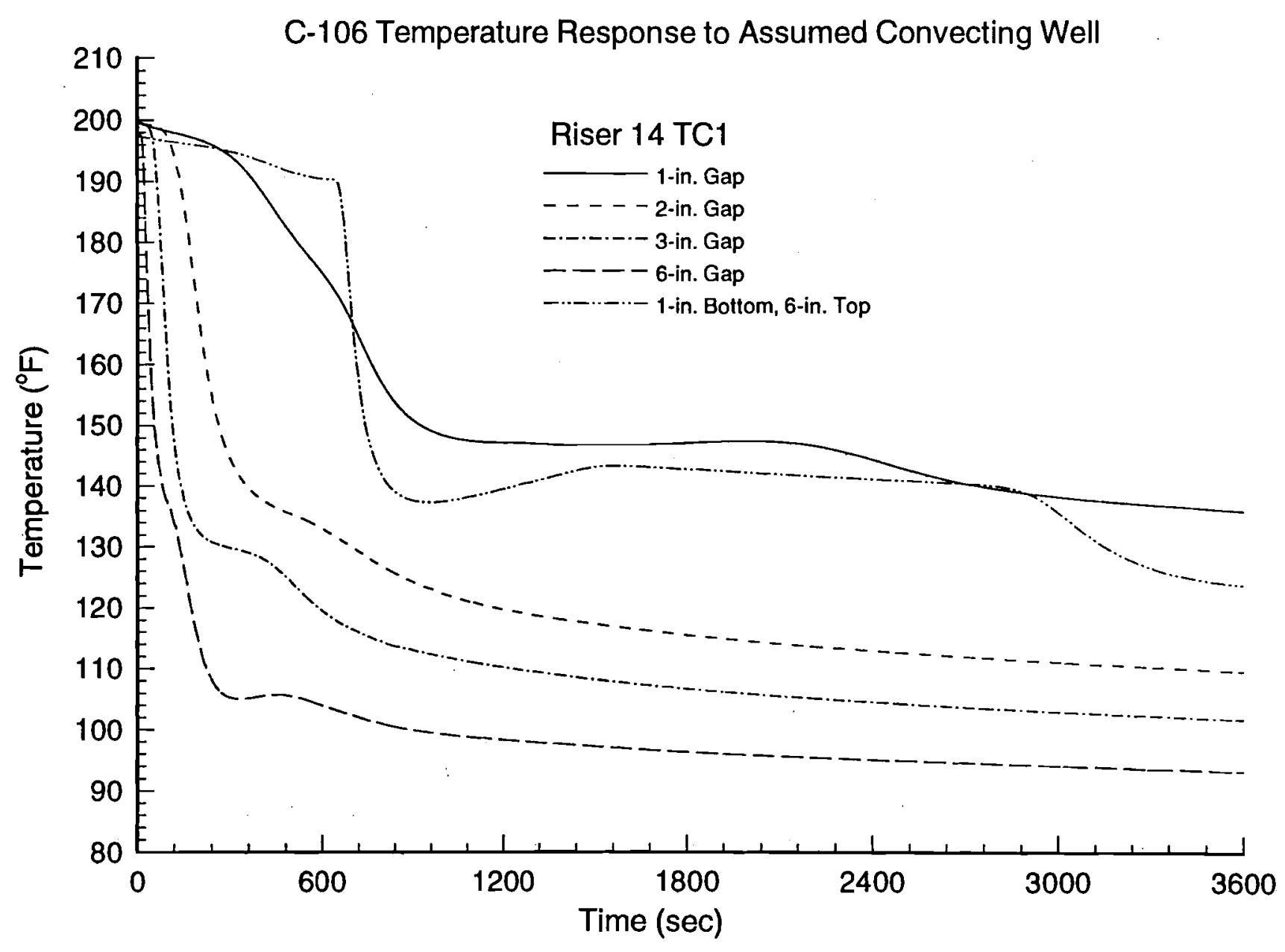

Figure 5.4. Riser 14 TC1 Response to an Assumed Convecting Well 


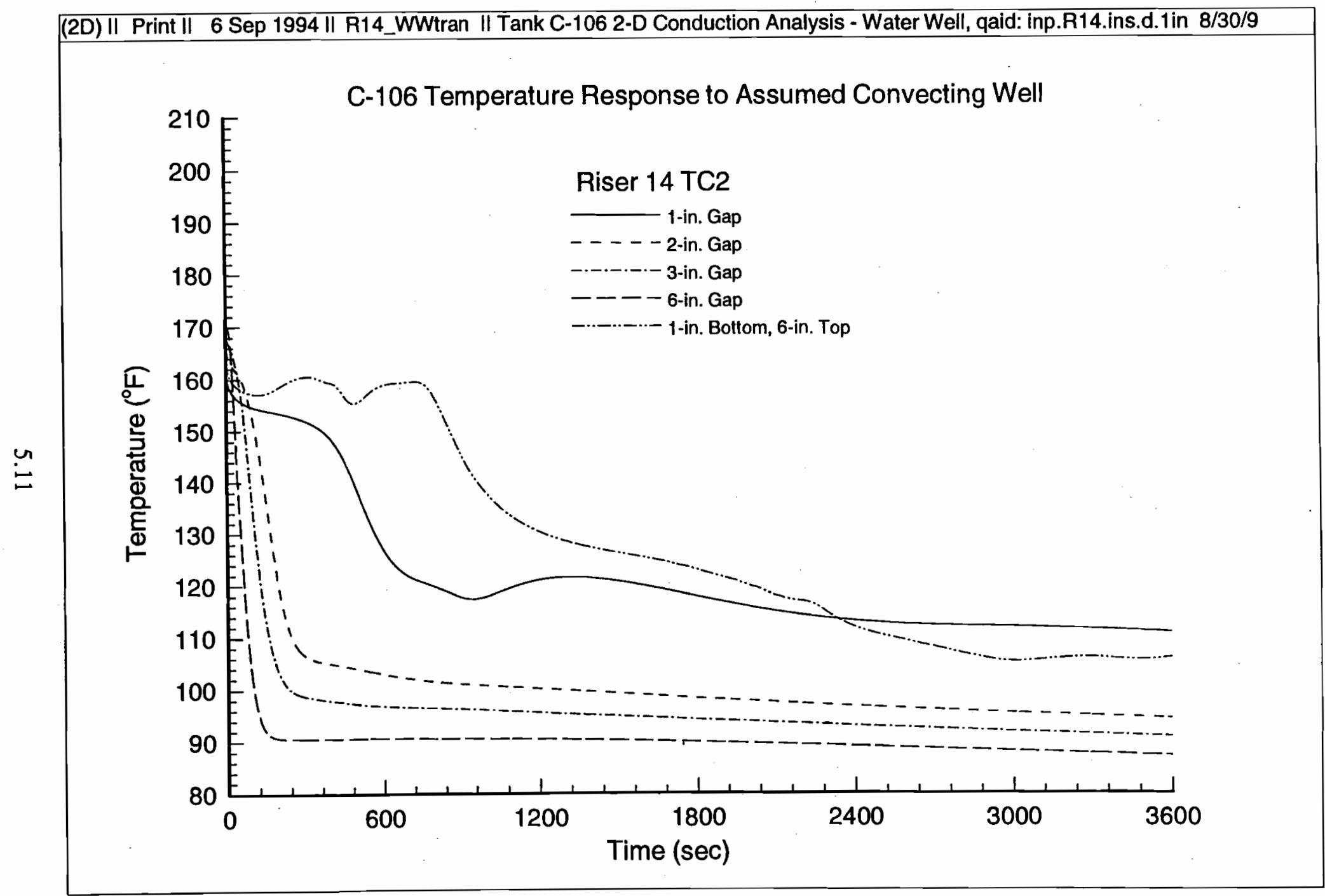

Figure 5.5. Riser 14 TC2 Response to an Assumed Convecting Well 


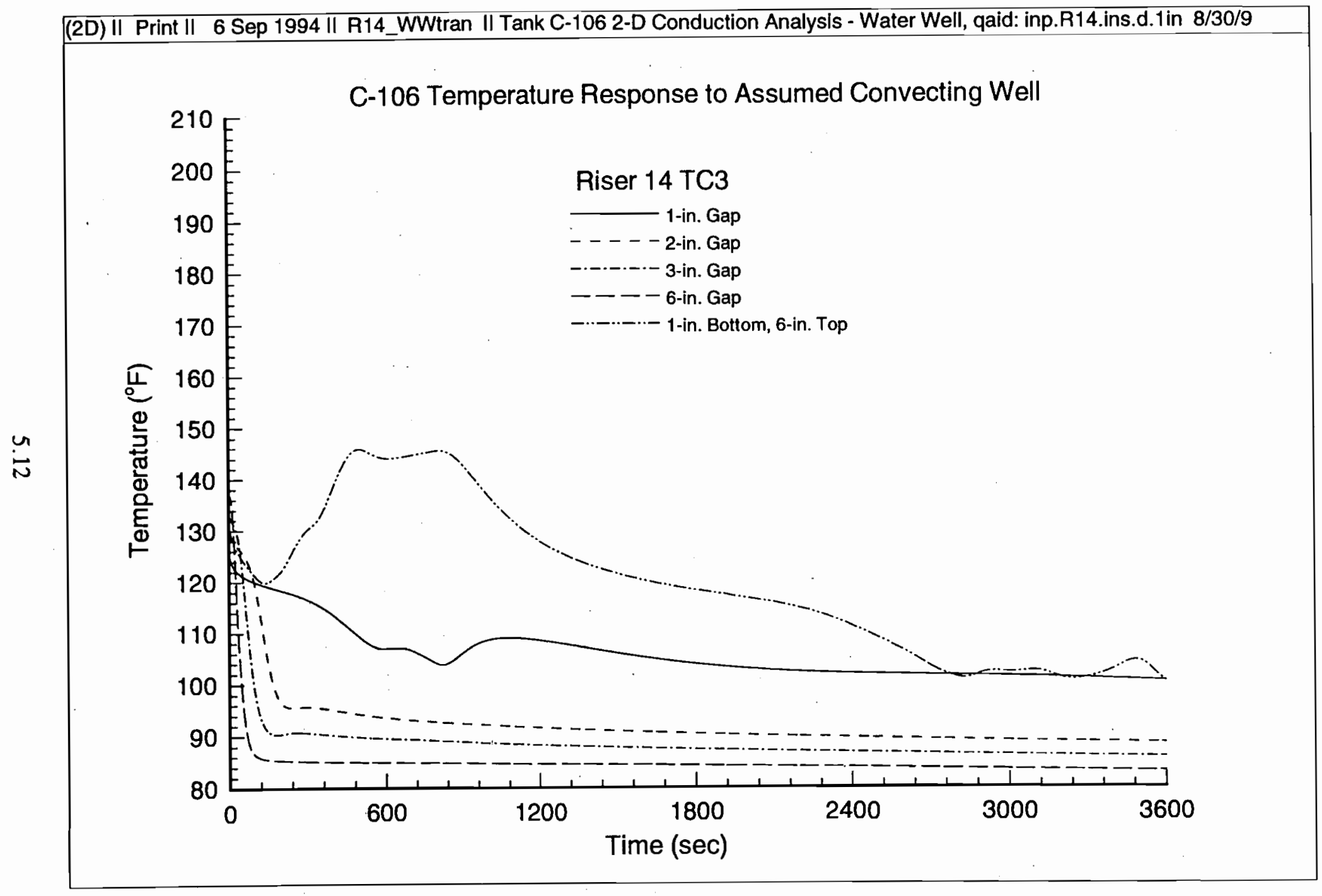

Figure 5.6. Riser 14 TC3 Response to an Assumed Convecting Well 


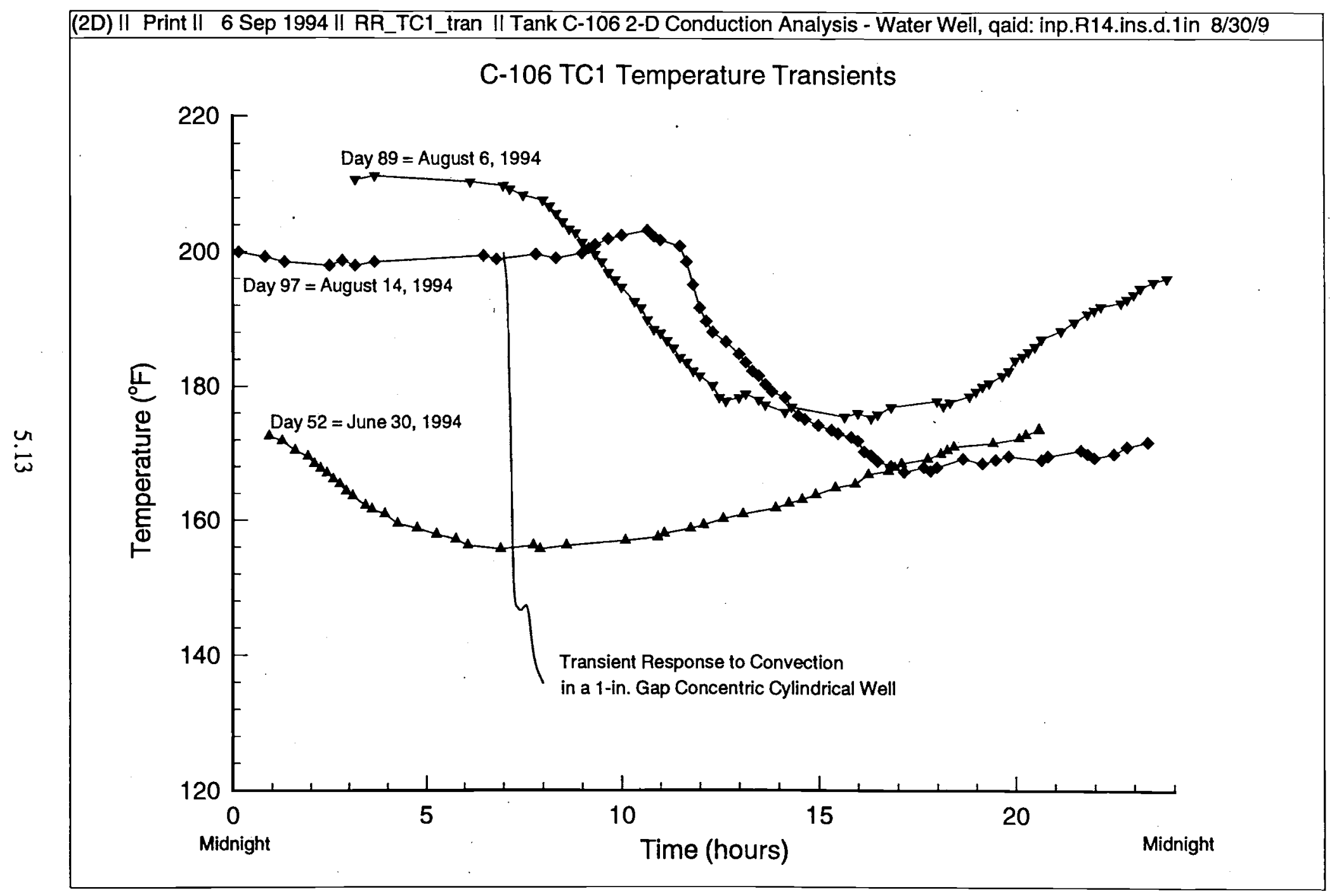

Figure 5.7. Comparison of Response of Convecting Well to Observed Temperature Indication Transients 
$r-z$ plane at $I=1$

$J=2$ to 49

$K=2$ to 33

plane $\min =8.000 E+01$

plane $\max =2.131 E+02$

array $\min =6.753 E+01$

array $\max =2.131 E+02$

$-12-2.000 E+02$

$-11-1.900 E+02$

$-10-1.800 E+02$

- $9-1.700 E+02$

- $8-1.600 E+02$

$-7-1.500 E+02$

$-6-1.400 E+02$

$-5-1.300 E+02$

- $4-1.200 E+02$

$-3-1.100 E+02$

$-2-1.000 E+02$

$+1-9.000 E+01$

Figure 5.8. Steady State Temperature Distribution Around Riser 14 for an Assumed 1-inch Convecting Gap Well 


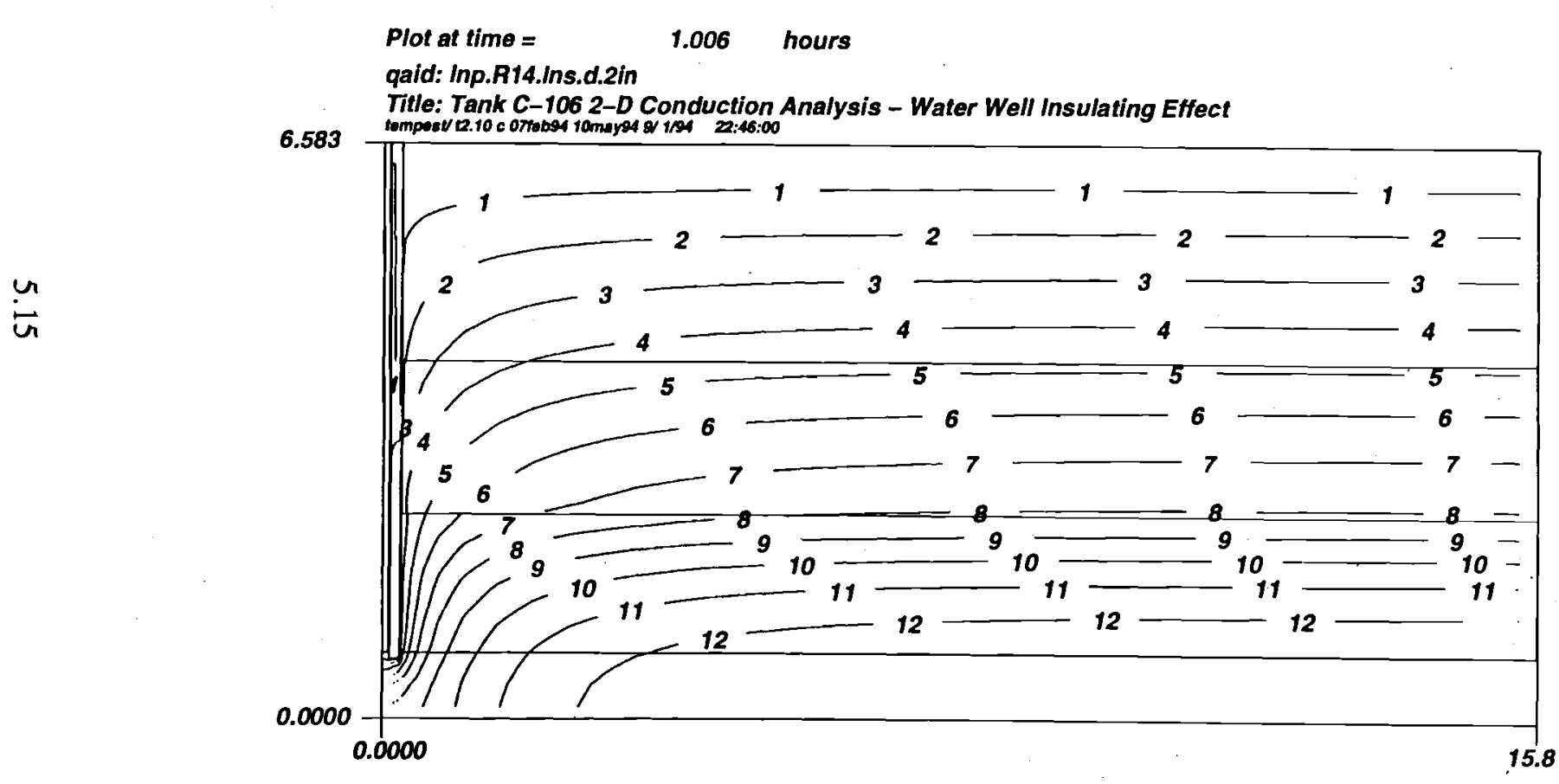

r-z plane at $l=1$

$J=2$ to 49

$K=2$ to 33

plane $\min =8.000 E+01$

plane max $=2.130 E+02$

array $\min =6.753 E+01$

array $\max =2.130 E+02$

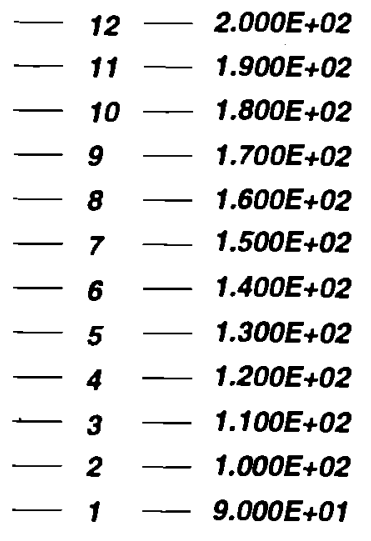

Figure 5.9. Steady State Temperature Distribution Around Riser 14 for an Assumed 2-inch Convecting Gap Well 
$r-z$ plane at $I=1$

$J=2$ to 49

$K=2$ to 33

plane $\min =8.000 \mathrm{E}+01$ plane $\max =2.129 E+02$

Plot at time $=$

1.018 hours

qaid: inp.R14.ins.d.3in

Title: Tank C-106 2-D Conduction Analysis - Water Weil Insulating Effect

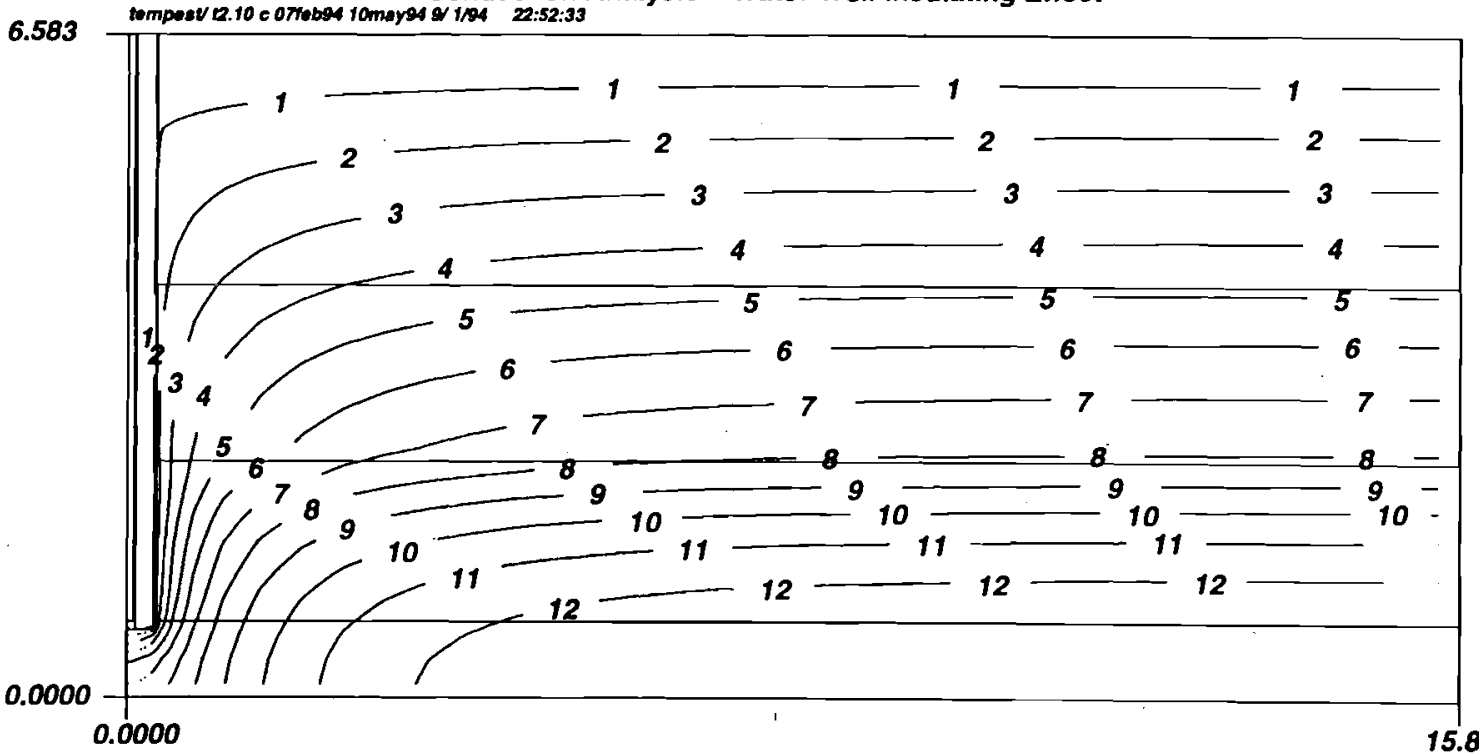

array $\min =6.753 E+01$

array $\max =2.129 E+02$

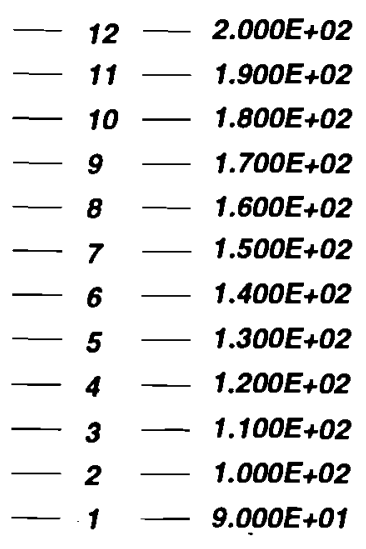

Figure 5.10. Steady State Temperature Distribution Around Riser 14 for an Assumed 3-inch Convecting Gap Well 


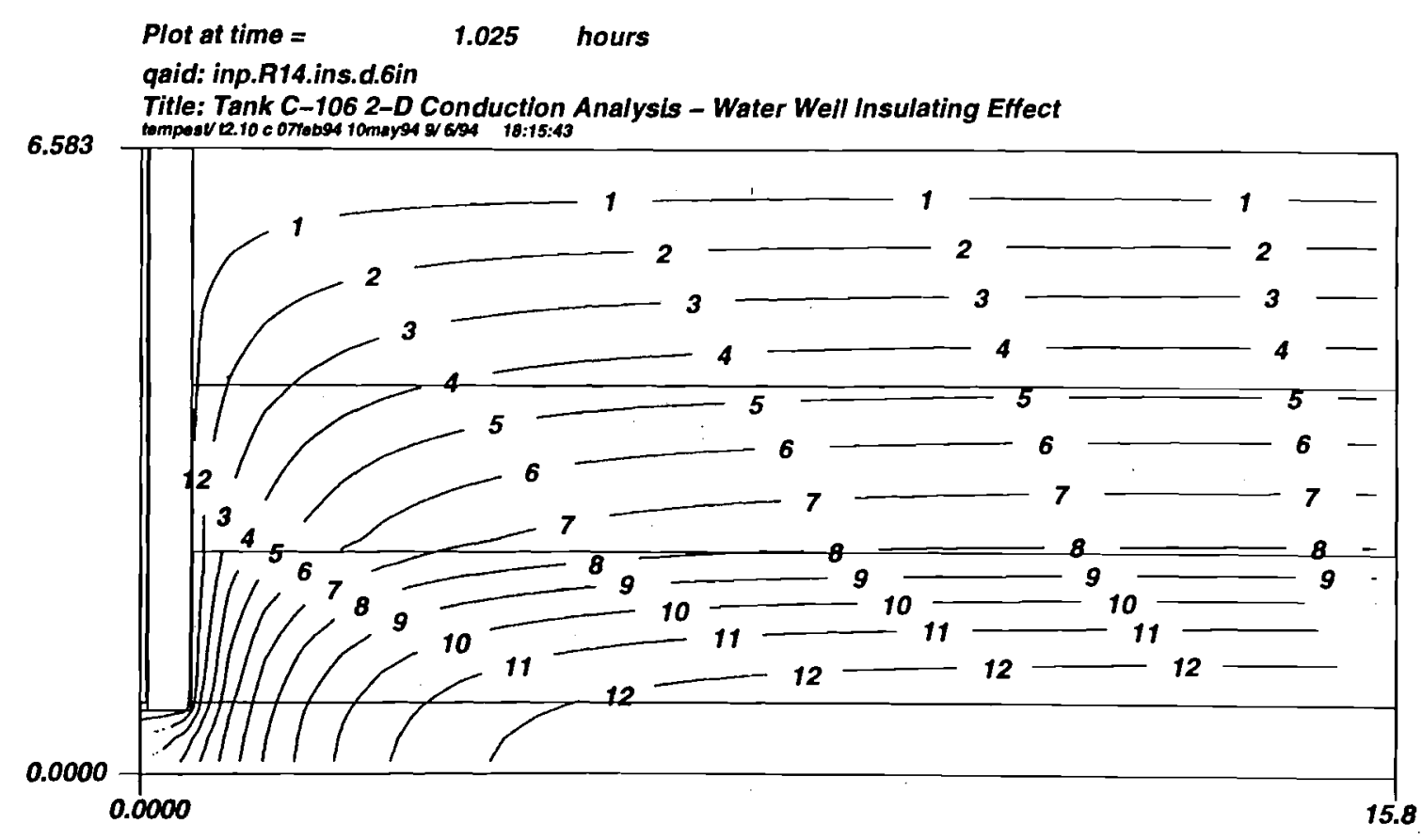

$r-2$ plane at $I=$
$J=2$ to 49
$K=2$ to 33

plane $\min =7.986 \mathrm{E}+01$ plane $\max =2.128 E+02$ array $\min =6.753 E+01$ array $\max =2.128 E+02$

Figure 5.11. Steady State Temperature Distribution Around Riser 14 for an Assumed 6-inch Convecting Gap Well 


\section{$r-z$ plane at $I=1$ \\ $J=2$ to 49 \\ $K=2$ to 33}

plane $\min =7.980 E+01$ plane $\max =2.113 E+02$ array $\min =6.753 E+01$ array $\max =2.113 E+02$ qaid: inp.A14.1in.1-6.a

Title: Tank C-106 2-D Conduction Analysis - Conical Water Well

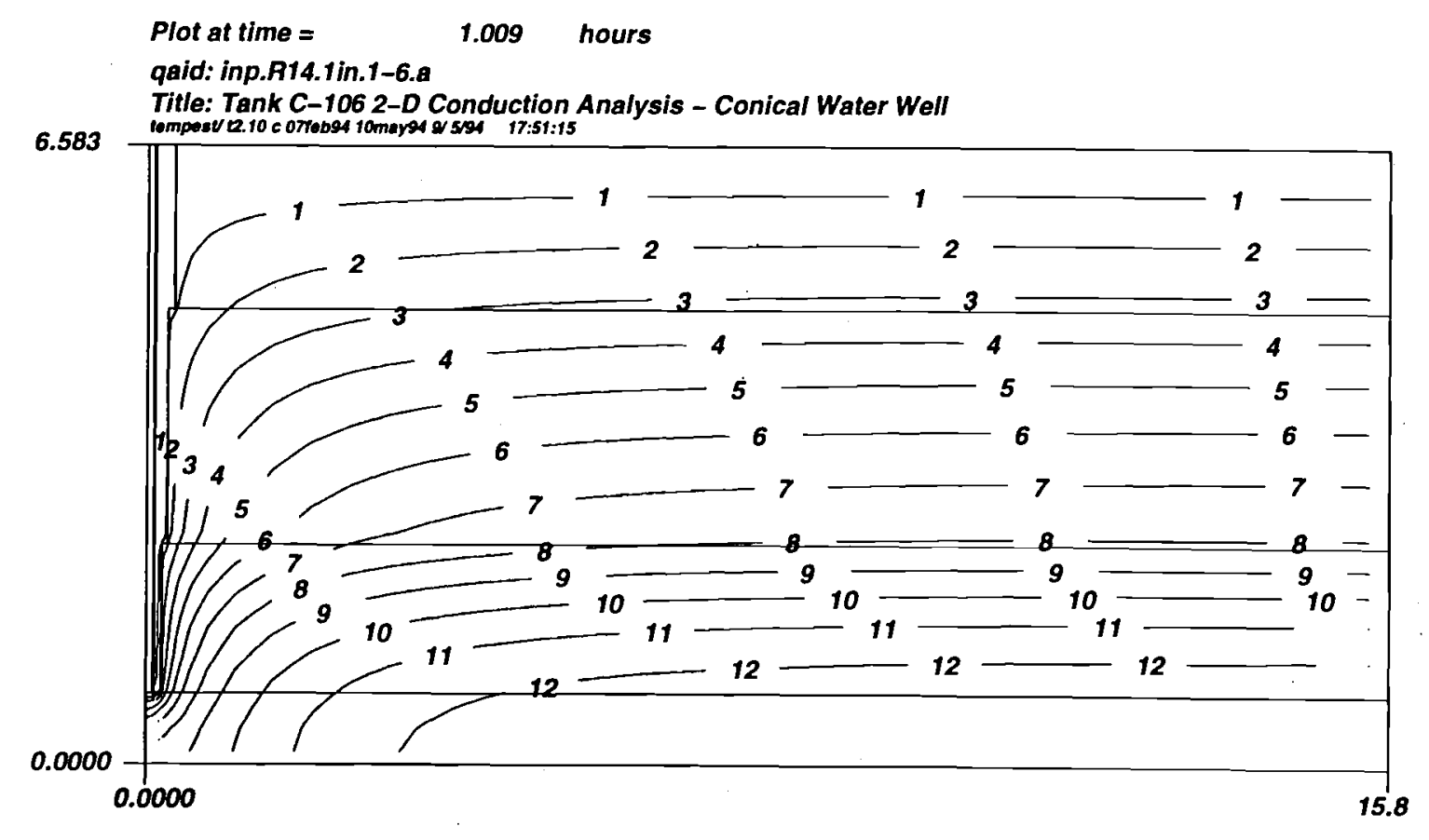

$-12-2.000 E+02$

$-11-1.900 E+02$

$-10-1.800 E+02$

$-9-1.700 E+02$

$-8-1.600 E+02$

$-7-1.500 E+02$

$-6-1.400 E+02$

$-5-1.300 E+02$

$-4-1.200 E+02$

- $3-1.100 E+02$

- $2-1.000 E+02$

$1-9.000 E+01$

Figure 5.12. Steady State Temperature Distribution Around Riser 14 for an Assumed Conical Shaped Convecting Gap Well 



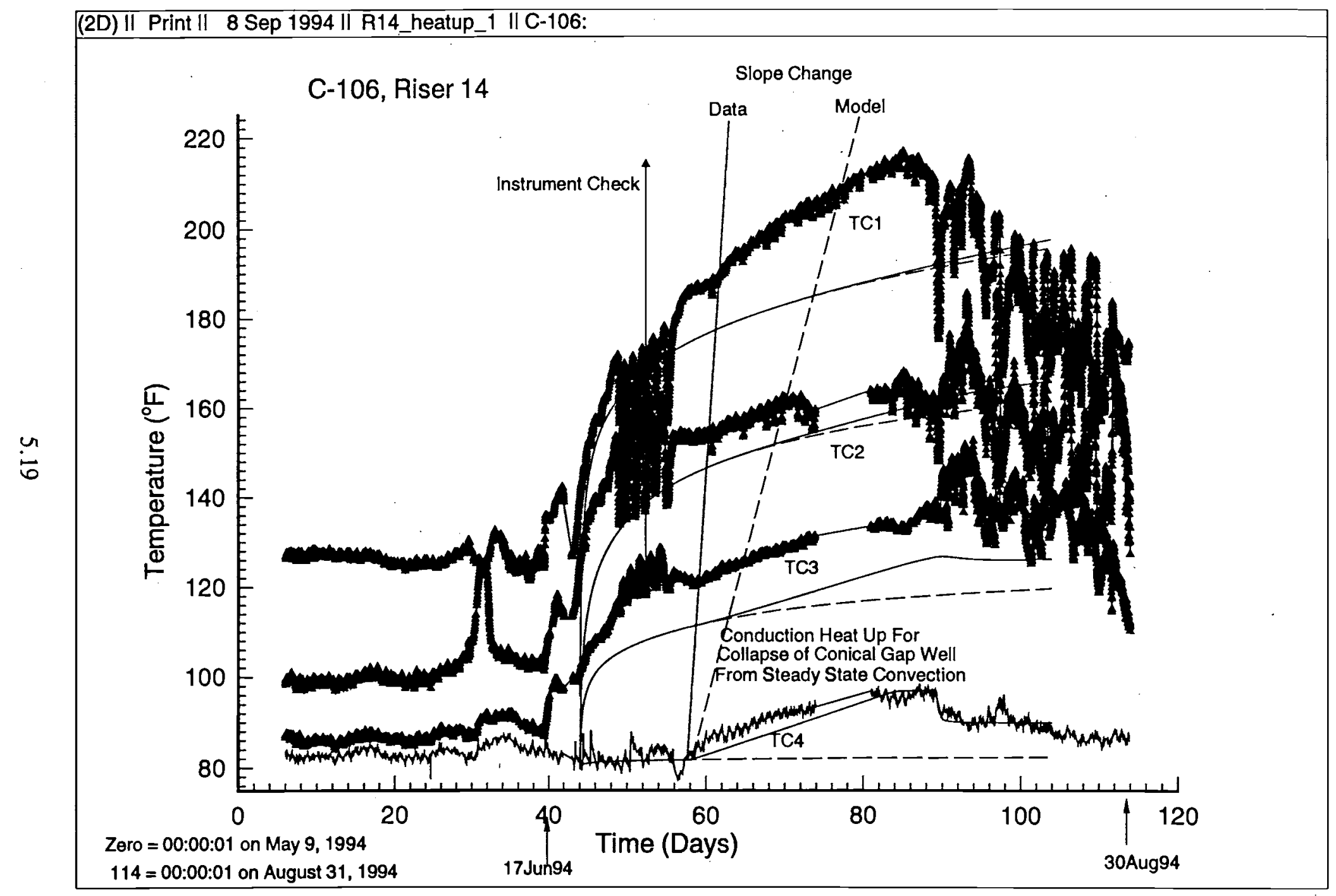

Figure 5.13. Comparison of Riser 14 TCs to Assumed Conduction Heat Up in Response to Loss of Convecting Gap Well 


\subsection{Temperature Response Due to Assumed Sludge Motion}

In this section, an narrative explanation of the indicated temperature response of riser 14 is offered. It is based on information gathered from several sources:

- observations taken from the historical indicated temperature response data in the SACS and TMACS databases

- hypotheses as to the nature of the physical mechanisms that could lead to the indicated response

- time response analyses conducted and presented in the previous sections

- analyses completed that identify certain mechanisms but that cannot explain observed response

- discussions with and analyses conducted by other investigators involved with the C-106 Temperature Response Team (principally the Red and Green Team members)

- theories offered by others in group discussions and brainstorming sessions

- convection and conduction analyses performed to support the explanation.

To present a case or explanation for the observed temperature response at riser 14 , it is necessary to assume the temperature indications in the thermocouples in riser 14 are in fact representative of temperature in the vicinity of riser 14 . While this assumption is not irrefutable, sufficient explainable, consistent trends appear to be included in the data to warrant their consideration. Furthermore, instrumentation checks have found no identifiable reason that temperature indications are erroneous, based on instrumentation. ${ }^{(a)}$

The explanation offered in this report is not definitive, but it does offer a framework within which virtually all the observed characteristics are identified.

\subsection{A Case for Sludge Motion - Narrative}

To develop a case for sludge motion as the basis for some of the indicated temperature responses observed at riser 14 , it is useful to present a narrative of the observations that lead to the conclusion. To do this, the ventilation outage period in late-January through earlyJune, 1992 is used as the starting point. During this outage, riser 8 temperatures indicated a

(a) C. C. Scaief. Presentation on C-106 thermocouples and instrumentation to the Chemical Reactions Sub-TAP, September 12, 1994. 
rather significant heat-up event with TC1 reaching a maximum temperature of about $200^{\circ} \mathrm{F}$. This heat up was rather linear and has been used as the basis for calibrating conduction models of tank C-106. During this heat up, no data are available from riser 14. However, the dome space temperatures, as indicated by TC5 and TC6 in riser 8, also show a rather marked increase. Given the heat rate in the tank and the conduction modeling analysis results, it is logical to conclude that a significant zone of sludge reached a saturation temperature. While more detailed analyses of this event are planned, until those analyses are completed, the state of the sludge is speculative (whether a dried out zone developed, for example). Estimates of the time required to dry out the whole tank vary from 5 to 17 months, depending on assumed water content fraction ${ }^{(a)}$.

Independent of whether dry out occurred, upon return to normal water fill and evaporation cycles, temperatures in riser 8 returned largely to pre-ventilation outage values (again without any noticeably significant temperature oscillations beyond nominal annual variations). From these observations, and, in fact, including data through 1991, it can be concluded that riser 8 is in a zone that is predominantly conduction heat transfer dominated. No temperature variations are observed in riser 8 data that would indicate any variations other than those expected from a thermocouple that is in direct contact with a consolidated or non-convecting sludge.

Riser 14 data were recorded on a more regular basis beginning in early 1993. Temperature indications exhibited a significant amount of variability, about 20- to 30-degree variations in temperature. Curiously enough, close examination of TC3 on riser 14 shows that its temperature is approximately the same temperature as TC4 over some periods of time and approximately the same as TC2 at other times during most of 1993. A close correlation of indicated temperature at TC3 between water additions $s^{(\mathrm{b})}$ appears during this period of time. Thus, when larger quantities of water were added in 1993, temperatures at TC2 and TC3 increased. Such a temperature increase leads to the conclusion that heat transfer around riser 14 (at least the upper two-thirds of it) is accomplished by some convective heat transport mechanism and furthermore, that heat transfer around riser 14 cannot be by a conductive mechanism. If this situation were not the case, its indicated temperature would be more closely approximated (in both time response and magnitude) with conduction heat transfer computer modeling.

The last direct correspondence response of riser $14 \mathrm{TCs}$ to water addition, prior to the process test, is February, 1994, as shown in Figure 6.1. Unfortunately, the data in March of 1994 , the first month of the process, is very scant, with only one temperature indication

(a) H. C. Reid, September 1994. "Tank C-106 Assessment of Local Sludge Dryout." ASD/HCR94-002, Rev. 1. Pacific Northwest Laboratory, Richland, Washington. (Internal Letter Report to Westinghouse Hanford Company.)

(b) For just that reason, the temperature increase that was observed in TC3 of riser 14 following the water addition on September 9, 1994, could have been predicted, or, at least, anticipated. 
recorded at mid-month. Data were recorded much more regularly beginning in April. More frequent recordings became available with the on-line TMACS data which began in early May.

During the process test, from March 4 through early June, 1994, TC3 and TC4 on riser 14 indicate temperatures that are in close agreement with each other. This observation, coupled with 1993 data, leads to several observational conclusions. First, TC3 (probably at least a foot below the nominal sludge/liquid interface) is in good convective thermal communication with surface liquid. Axial conduction in the riser 14 thermocouple tree cannot explain this low temperature in TC3. Second, TC4 (most likely in a position about equal to an FIC reading of 77 inches and, therefore, uncovered for most of the process test) is approximately 10 to $12^{\circ} \mathrm{F}$ cooler than TC4 on riser 8 . This difference indicates TC4 on riser 8 is either significantly lower and perhaps not in the liquid, or the sludge depth around riser 8 is quite a bit higher than at riser 14 , or the axial conduction effects in riser 8 are significantly greater than in riser 14. From video results, it is most likely the nominal sludge depth around riser 8 is greater than around riser 14. From analysis of thermocouple locations, the TC4 on riser 8 is lower than TC4 on riser 14. Both effects compound the fact that TC4 on riser 8 indicates higher temperatures than on TC4 on riser 14. The further conclusion that can be drawn from this observation is that TC4, when the FIC surface liquid level indication is in the 77- to 79-inch range, is a better indication of surface liquid temperature. At this point, a $10-12^{\circ} \mathrm{F}$ horizontal variation in surface liquid temperature distribution cannot be discounted.

From this discussion, it is apparent that historically some sort of enhanced heat transfer mechanism exists, at least in the upper region (TC3 and above) around riser 14. The theory that riser 14 exists in a well has been offered repeatedly. If such a well existed prior to the end of the process test about mid-June, it would have had certain physical characteristics, perhaps of a conical shape. Complete convective communication through a large well (greater than a 1-inch gap annular region) extending to the bottom of riser 14 can be shown by convection analysis as incapable of supporting the axial temperature gradient indicated by $\mathrm{TC} 2$ and TC1. Thus, for convective communication to exist below TC3, the gap would have to be very small, the liquid in the gap would have to be very viscous or particle laden, and the gap would have to be surrounded by consolidated or non-convecting sludge (that is, have some strength). With this picture of the character of the well, it is necessary to explain why TC3 temperature increases occurred in conjunction with water additions back in 1993.

At this point, it should be repeated that model analysis results have indicated a saturation zone probably developed during the 1992 ventilation outage. Evaluation of surface level variation as an indication of evaporation shows that removal of the excess energy in the tank took about five months. Furthermore, conduction model analysis results also indicate the normal state of the tank may have had a saturation zone, even after the excess energy had been removed. It is quite possible a saturation zone existed through 1993. If such a saturation zone did exist, its presence becomes the explanation for the temperature oscillations observed in riser 14 TCs. 
Assume a saturation zone somewhere in the tank. If it exists, it is most likely in some central location near the floor. It could, in fact, be in the salt cake that might exhibit a consolidated characteristic of a porous media. When water is added to the tank, the hydrostatic pressure at the bottom of the tank increases accordingly. This increase requires some continuous liquid communication path from the surface to the tank floor in some region outside the radius or extent of the saturation zone. The liquid addition would also increase the hydrostatic pressure on the surface of the saturation zone. Thus, the increased hydrostatic pressure has two effects. The first is to correspondingly increase the saturation temperature, which would tend to collapse the saturation zone. However, this process is time-controlled by the ability of liquid to permeate to the saturation zone boundary to replace the volume of steam after condensation. Second, the increased hydrostatic pressure at the bottom of the tank outside the saturation zone would provide an additional pressure gradient to push liquid laterally to the side of the saturation zone. These two effects can arguably cause either a net increase or a net decrease in the size of the saturation zone. The timecontrolling processes of both the heat transfer and the liquid micro-convection in the vicinity of the saturation zone boundary are complicated. This effect has in fact been hypothesized by others. ${ }^{\text {(a) }}$ On one hand, if the effect of adding water caused a net increase in the saturation zone size, it could partly explain the net decrease in the historical rate of surface level change following each water addition.

The other effect that either of the net changes in the saturation zone size has with a water addition is to cause sludge motion. These changes could be manifested by either a net upward swell (that confuses the surface level indications) or it could more locally cause liquid convection in surrounding media that are more like a consolidate particle/liquid material than a flowing, convecting sludge. It can be concluded from the photographs taken of the 1986 core sample, that both consolidated and flowable sludge material may be present in this tank. Another possible effect would be that a saturation zone steam void could develop in a flowable material region. In this case, the buoyancy effects could also induce convective sludge motion. Furthermore, sludge motion could have the effect of overcoming material strength in regions where the strength of the sludge, which could increase with time, is not too great.

Having concluded that a saturation zone steam void effect could induce motion in the sludge, it is useful to re-examine the temperature response of riser 14 . If steam-void-induced motion occurs, the temperature response of TC2 and TC3 in riser 14 during 1994 could logically be a result of either a net sludge motion or an effect of liquid movement through a surrounding consolidated material. If net sludge motion were the culprit, the motion would not need to be large to effect the magnitude of temperature changes observed. This conclusion is supported by examining computer modeled quasi-steady temperature fields in an assumed sludge field surrounding riser 14 . The motion would only need to be the size of the gap of

(a) M. J. Thurgood, Numerical Applications Incorporated, personal communication and Thurgood et al. (1994) 
the convective cooling well surrounding the thermocouple tree; the movement would only need to be enough to close the thin gap and move consolidated material into direct contact with the wall of the thermocouple tree. For this to occur, the nature of the consolidated material need only be of a rheological characteristic that has some amount of strength or cohesiveness. Many kinds of tank sludge exhibit such characteristics.

At this point, it is necessary to come back to beginning of the process test in March of 1994 . The last water addition was made at the first of March. From that time, until the sludge was largely uncovered in mid- to late-May, the tank was in good equilibrium, as indicated by the steady decrease in surface level. From March to May, one of two things must have happened. Either the saturation zone that had been present through 1993 had been completely eliminated through the natural course of events, or the saturation zone was present at the beginning of the process test in early March. It is possible the effect of decreasing liquid level, due to evaporation, caused the equilibrium saturation zone to increase in size proportional to the decrease in local hydrostatic pressure and saturation temperature. If the saturation zone were present throughout the process test and was growing proportional to the decrease in hydrostatic pressure, it would seem likely the surface level change would not have indicated such a historically consistent rate of decrease as to equal normal evaporation rate. From that observation, it seems more likely the steam void had been completely condensed. At a minimum, the steam void could have been confined to a small region in a consolidated porous media-type area, such as in the hard pan, and the porous media flow in the hard pan affected maintenance of a quasi-steady equilibrium size of saturation zone.

In the scenario where the saturation zone had been eliminated, it is straightforward to conclude that after the sludge had been uncovered sufficiently to reduce evaporative cooling, a net amount of energy was starting to be stored in the sludge. This net storage could have caused the saturation zone to re-form, causing a volume expansion and corresponding sludge movement. Such behavior could have resulted in the temperature perturbation observed in TC2 (and to a much lesser degree in TC1) of riser 14 in the June 9 and 10 time period, nearly seven days prior to re-adding water on June 17. Correspondingly, the same effect would occur if the saturation zone were present at the beginning of the process test and responded in a similar growth manner when evaporative cooling was reduced with sludge uncovered.

The nature of the observed temperature perturbation in TC2 on June 9 and 10 is such that it can only be explained by hypothesizing a net lateral movement through the middle of the sludge. The size of the movement would only need be large enough to reduce or eliminate any thin gap surrounding the tree. The return of the temperature indication of TC2 to its previous state indicates movement of a sludge that exhibits characteristics of an elastic or cohesive rheology. Subsequently on June 17, water was added to the tank and temperatures of all three thermocouples in the sludge increased simultaneously with the water addition. 
Interestingly enough, no surface level change was observed with the June 17 water addition. It was possible that when the sludge was uncovered, the June 17 water addition was of insufficient quantity to cover the sludge to a level where the FIC surface level indicator could respond. The temperature rise that accompanied the June 17 water addition was of a time response nature quite different than previously observed increases with water additions. Correspondingly, a very similar simultaneous response from all three thermocouples occurred in the sludge with the June 21 water addition (for which an FIC level indication appeared after the addition). Computer analysis results of these two temperature rise events are typical of sludge movement that comes into contact with the pipe wall, followed by a conduction heat-up event of consolidated sludge surrounding a previously cooled local domain. These two events, although of a different time constant than that of certain other events, are consistent with hypotheses of sludge motion.

Subsequent to the second water addition of June 21 , temperature indications on all three thermocouples in the sludge began increasing, consistent with a computer model of a conduction heat-up process. This process would occur in consolidated material from the local sludge environment that had reached quasi-thermal equilibrium around a convectivecooled well at riser 14 . Up to this point, the indicated temperature response of riser 14 , due to hypothesized sludge motion, has indicated temperature increases with each initial movement and a corresponding decrease at a later time.

Note that TC4 on riser 14 also showed a temperature increase beginning shortly after the 14,000 gallon water addition of July 3 . This water addition brought the tank FIC level back up to 79 inches, just above the place where TC4 is expected to be located. Comparing the indicated temperature response of TC4 in July 1994 with TC4 temperature response in the July to September period in 1993 shows they are of very similar magnitude.

Two more characteristic temperature indication responses need explaining. One concerns the six quasi-periodic oscillations that occurred just prior the 14,000 gallon water addition of raw water started on July 3, 1994; the other is the more chaotic nature of oscillations observed during the entire month of August.

The quasi-periodic oscillations have very unusual features that have not yet been fully explained. It appears at first glance (depending upon which figure is observed) the initial response is downward-trending temperatures. All three thermocouples seem to be in phase and tracking each other. The period grows with each oscillation, starting near 21 hours and ending near 34 hours. Expanding these oscillations in time leads to the identification of further coherency. The oscillations are presented in an expanded scale in Figure 6.2. Within each oscillation, definite correspondence exists with identifiable changes in the slope of the temperature indications. It is useful to note that TC2 always seems to be the leader. It even appears the first temperature response is in fact an upswing, but with a slightly slower response time than other movements discussed previously. Several of the corresponding features are noted by lines drawn vertically in Figure 6.2. Relative to the general trend of the indications before and after the oscillations, TC1 appears to oscillate 
below its eyeball average temperature; TC2 appears to oscillate around its eyeball average; and TC3 appears to oscillate above its eyeball average.

The only explanation for the character of these six periodic oscillations is some sort of quasiperiodic sludge movement is occurring and the movement is occurring in some sort of elastic or cohesive rheological material. With regard to the steam void, it could be the driving force, but it would necessarily be a rather periodic type of growth and collapse cycle. A more complete explanation and simulation analysis is still needed.

Note the periodic oscillations ceased with the completion of the addition of the 14,000 gallons of raw water started on July 3 . This addition would significantly increase the hydrostatic pressure at the tank bottom. Conceivably, this pressure increase caused a saturation zone to collapse, hence removing the steam void as a driving force for sludge movement. The temperature response indication for the period between July 4 and August 1 is symptomatic of a further conduction heat-up process. If it had collapsed with the July 3 water addition, the steam void could have reformed during this period.

After reaching peak temperatures on August 1, the indicated temperatures of riser 14 show a decreasing trend. After August 5, the indicated temperature oscillations exhibit a more chaotic character. Peak-to-peak oscillations as great as $30^{\circ} \mathrm{F}$ are observed, some of which are initially down-spikes. Again hypothesizing the steam void is the driving force, temperature oscillations are indicative of a bottom-heated, physically unstable, convective mixing process (albeit very slowly). The physically unstable nature during this time frame lies in the steam bubbles either being formed in a sludge region, or the net process of the steam bubble formation is causing a degradation of any cohesiveness or strength. The resultant sludge motion could overcome any cohesiveness or strength of any material in the direct vicinity of riser 14 . The net downward temperature trend of all three thermocouples in the sludge is indicative of a general convective process and a cooling process that would result from the more efficient heat transfer due to sludge movement.

This narrative emphasizes that formation, presence, or collapse of a saturated boiling process somewhere in the lower, central region of the sludge is inducing (or has induced) sludge motions that can explain the observed temperature indications in riser 14 . While this narrative is not definitive, it offers a sequentially consistent explanation of the events that could have occurred as a result of the process test. 


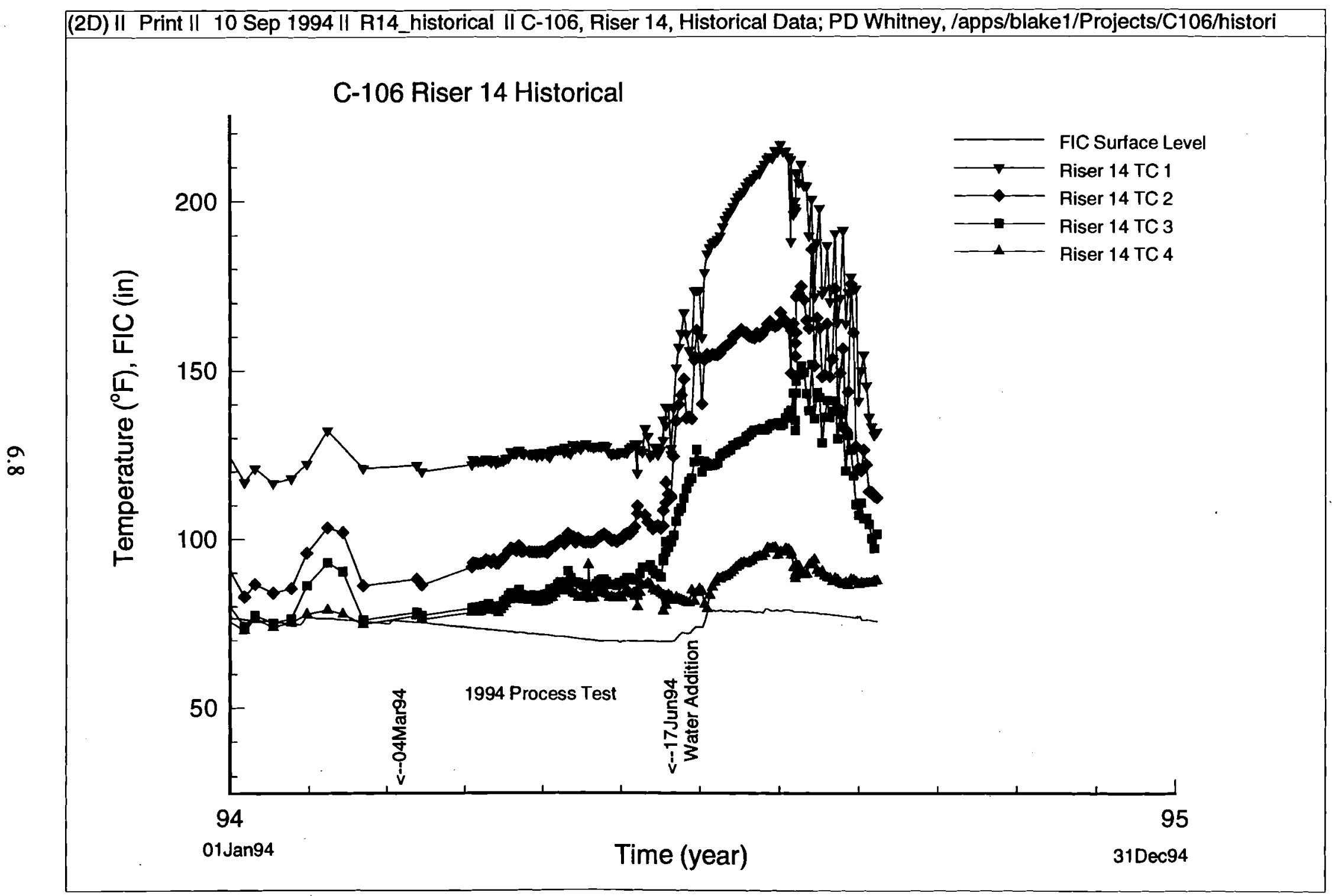

Figure 6.1. Riser 14 Temperatures - SACS Database, January through August 1994 


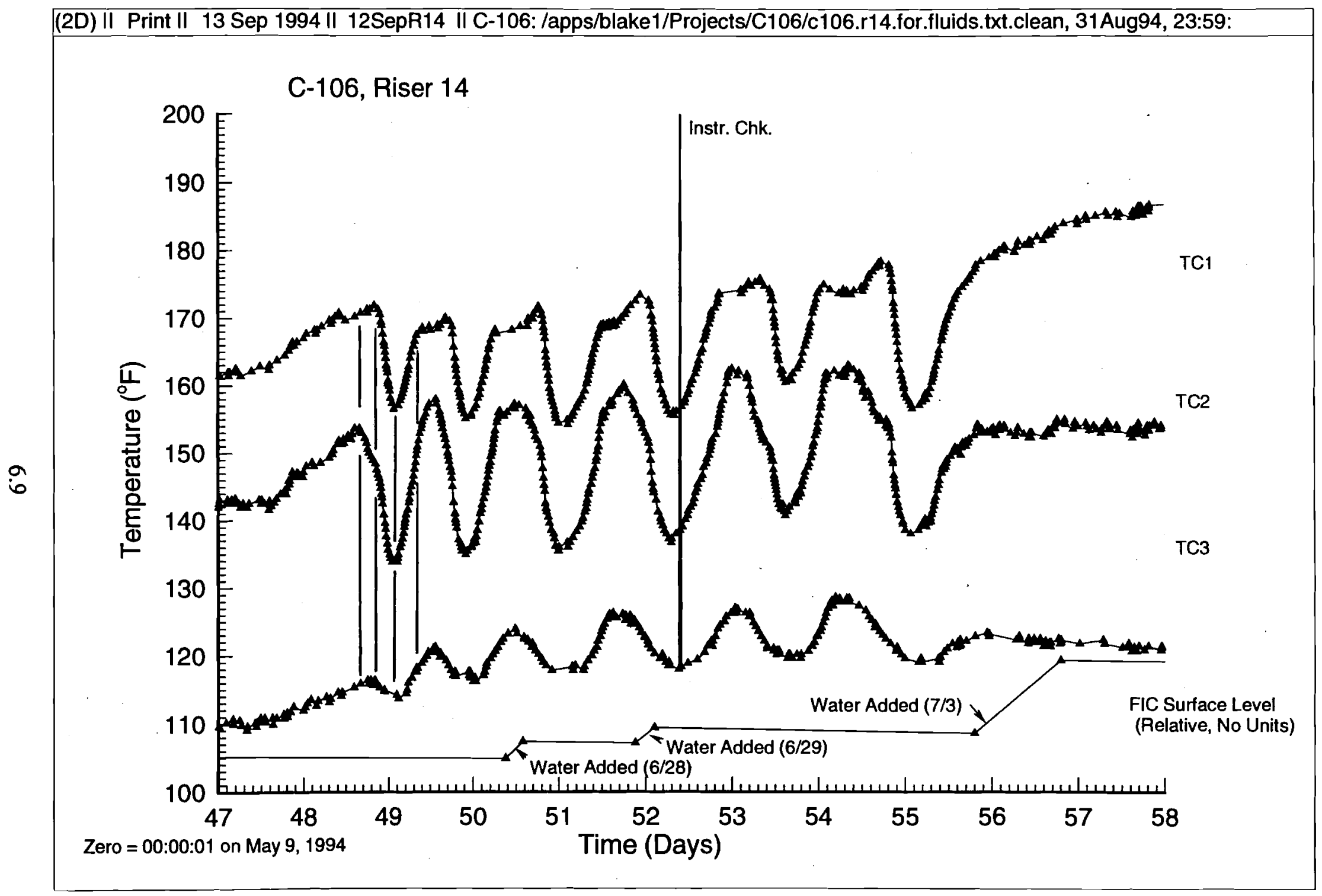

Figure 6.2. Riser 14 Temperatures - TMACS Database, Late June 1994, Showing Six Quasi-Periodic Oscillations 



\subsection{References}

Bander, T. J. 1993. Revised Thermal History of Tank 241-C-106. WHC-SD-WM-ER-200, Rev. 0, Westinghouse Hanford Company, Richland, Washington.

Bander, T. J., and B. Crea. 1994. An Assessment of the Potential for a Steam Bump in Hanford Waste Tank 241-C-106. Westinghouse Hanford Company, Richland, Washington. (C-106 Temperature Response White Paper dated August 6, 1994).

Marks' Standard Handbook for Mechanical Engineers, Eighth Edition, McGraw-Hill, New York, 1978, pages 4-61.

Thurgood, M. J., B. C. Fryer, and S. W. Claybrook. 1994. Preliminary Report Tank 106C Thermal Hydraulics Analysis Related to the March 10 - June 15, 1994 Process Test and Temperature Rise of Riser 14 Thermocouples. NAI-940708-1, Numerical Applications Incorporated, Richland, Washington.

Trent, D. S., and L. L. Eyler. 1993. TEMPEST - A Computer Program for ThreeDimensional Time-Dependent Computational Fluid Dynamics. PNL-8857, Pacific Northwest Laboratory, Richland, Washington. 


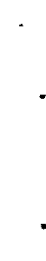




\section{Appendix}

Supporting Information 


\section{Appendix}

\section{Supporting Information}

This appendix contains several referenced memos and certain data that have not been widely distributed. They are included here in their original state for completeness.

A. 1 


\begin{tabular}{|c|c|c|c|c|}
\hline Date & CASS Log Entry & $\begin{array}{l}\text { gat. } \\
\text { added }\end{array}$ & $\begin{array}{l}\text { Star } \\
t \\
\text { Time }\end{array}$ & $\begin{array}{l}106-C \\
\text { FIC } \\
\text { level } \\
\text { change } \\
\text { (in.) } \\
\end{array}$ \\
\hline \multirow[t]{3}{*}{$6 / 17$} & 1025: Adding water to $106-C$ & \multirow{3}{*}{$\begin{array}{l}2347 \\
(.853 ") \\
(R W \text { add. } \\
\text { data } \\
\text { sheet) }\end{array}$} & \multirow[t]{3}{*}{1025} & \multirow{3}{*}{$\begin{array}{l}0.1 \\
\text { to } \\
69.5\end{array}$} \\
\hline & 1253: Stopped adding water to $106-C$ & & & \\
\hline & $\begin{array}{l}\text { 1335: Started adding water to } 106-C \\
\text { again }\end{array}$ & & & \\
\hline \multirow[t]{3}{*}{$6 / 22$} & 0920: Water being added to $106-C$ tank & \multirow{3}{*}{$\begin{array}{l}1749 \\
\left(.636^{\prime \prime}\right) \\
\text { (CASS } \\
\text { Log) }\end{array}$} & \multirow[t]{3}{*}{0920} & \multirow{3}{*}{$\begin{array}{l}1.0 \\
\text { to } \\
70.6\end{array}$} \\
\hline & 1205: Water has stopped at $106-C$. & & & \\
\hline & $\begin{array}{l}\text { 1330: Water addition to } 106-C \text { was } \\
1749 \text { gallons. }\end{array}$ & & & \\
\hline \multirow[t]{3}{*}{$6 / 23$} & $\begin{array}{l}\text { 0845: Begun adding water again to } \\
\text { 106-C. Level is at } 70.6 \text { now. }\end{array}$ & \multirow{3}{*}{$\begin{array}{l}2273 \\
(.827 ") \\
\text { (CASS } \\
\text { Log) }\end{array}$} & \multirow[t]{3}{*}{0845} & \multirow{3}{*}{$\begin{array}{l}0.7 \\
\text { to } \\
71.2\end{array}$} \\
\hline & 1135: Stopped water addition to $106-\mathrm{C}$ & & & \\
\hline & $\begin{array}{l}\text { 1455: } 2273 \text { gals of water was added to } \\
106-C \text { today. Level is at } 71.3\end{array}$ & & & \\
\hline \multirow[t]{2}{*}{$6 / 24$} & 0855: Operation adding water to $106-\mathrm{C}$ & \multirow{2}{*}{$\begin{array}{l}2122 \\
\text { (.772") } \\
\text { (RW usage } \\
\text { data sheet) }\end{array}$} & \multirow[t]{2}{*}{0855} & \multirow{2}{*}{$\begin{array}{l}0.6 \\
\text { to } \\
72.0\end{array}$} \\
\hline & $\begin{array}{l}\text { 1230: Operations shut the water } \\
\text { addition off to } 106-C \text {. FIC reading is } \\
72.3 \text {. }\end{array}$ & & & \\
\hline $6 / 28$ & $\begin{array}{l}\text { 0923: Notified that water is being } \\
\text { added to } 106-C \text {. }\end{array}$ & $\begin{array}{l}2706 \\
(.984 ") \\
\text { (6/27 RW } \\
\text { usage data } \\
\text { sheet) }\end{array}$ & - & $\begin{array}{l}1.1 \\
\text { to } \\
73.0\end{array}$ \\
\hline $6 / 29$ & $\begin{array}{l}\text { 2111: Started adding water to } 106- \\
\text { C...will add } 3575 \text { gal. or until level } \\
\text { reaches } 74.0 " \text {. }\end{array}$ & $\begin{array}{l}3075 \\
\left(1.118^{\prime \prime}\right) \\
\text { (RW usage } \\
\text { data sheet) }\end{array}$ & 2111 & $\begin{array}{l}1.1 \\
\text { to } \\
74.0 *\end{array}$ \\
\hline $7 / 3$ & & $\begin{array}{l}14425 \\
(5.246 ") \\
\text { (RW usage } \\
\text { data sheet) }\end{array}$ & 2000 & $\begin{array}{l}5.2 \\
\text { to } \\
78.8\end{array}$ \\
\hline
\end{tabular}

Then it fell back to 73.7 a few hours later. Maybe they stopped it when the first ripple went by and caused the level to read 74.0. 
Date: $\quad$ Sept. 9, 1994

To: $\quad$ Loren Eyler

From: Del Lessor

Subject: $\quad$ Separation Phenomena around Columns in Tank Waste

There are mechanisms which can produce an enclosing layer of almost pure liquid about a column, pipe, or probe inserted into a medium which contains solids suspended in a liquid. A layer of liquid formed by these processes could possibly occur around instrument trees inserted into the "gelled" region of a Hanford waste tank. Such a layer would probably allow more vertical convection near the instrument tree than would otherwise occur, and this should be kept in mind in interpreting temperature measurements from thermocouples on the instrument trees. The purpose of this note is to mention and discuss qualitatively some mechanisms that can lead to the formation of such a liquid layer.

When a compression or rarefaction front, either pulsed or oscillatory, travels in an inhomogeneous medium such as a suspension of solids or bubbles in a liquid, there is some relative motion of the suspended materials and the liquid. One can think of this in two intuitive ways. First, if the compression front requires motion of the material, suspended solids that are denser than the liquid possess more inertia and tend to lag behind the fluid motion. Alternatively, the compression front has an accompanying traveling pressure profile in the liquid, and the suspended denser solid experiences a net force toward the region of higher pressure, much like a solid experiences in a liquid whose static pressure profile is set up by a gravitational field.

Consider the consequences of this relative motion of solids and liquid under three scenarios:

1. If the number density and volume fraction of the suspended solids is low, the relative motion of liquid and solid from traveling periodic oscillatory compression or rarefaction waves produces primarily only an oscillatory position change of the solids.

2. If the oscillatory motion is a standing wave rather than a traveling wave, the solids tend to accumulate at the antinodes and bubbles at the nodes of the standing wave pattern, due to the time averaged effects of the oscillatory pressure gradient. This effect is used for degassification, and it could also be used for consolidation of solids. I think that this is strong effect for bubbles and a secondary effect for solids. The "rectification" effect is due, I think, to the difference in compressibility of the liquid and of the suspended material. The buoyancy-like net force during a compression part of a standing wave half-cycle is not exactly of equal magnitude and opposite sign to that during the corresponding rarefaction half-cycle, due to the density difference.

3. If the number density and volume fraction of suspended solids is high, even aperiodic pressure fronts and their associated material motion can result in consolidation of solids. This occurs if the forces at the molecular level give an attraction effect when the solids are brought into forceful contact.

The third of these three mechanisms is probably the one that contributes strongly to the 
formation of a solids-free layer around a probe, pipe, or instrument tree that experiences some motion relative to the inhomogeneous liquid-solid mix into which it is inserted. A transitory relative (of probe and liquid) acceleration and velocity of the probe in one direction results in an increase of the number density of solids at the surface on the side of the probe facing incoming liquid. The molecular level attraction forces produce a bonding together of solids, such that the resulting matrix does not follow the motion of the probe surface when the relative motion is reversed. A common example of this is enlarging a hole in water-saturated sand by wiggling a stick. After some period of time and many relative movements of probe and liquid, one might expect a nearly solids-free region to form with a thickness on the order of the larger relative displacements of probe and the surrounding medium. This phenomenon probably occurs even when the relative motion must first yield a region around the probe in material exhibiting the elastic solid to viscous fluid transition.

In a somewhat dynamic environment such as Tank 106-C, the solids-free layer might allow numerous possibilities for flow histories. For example, in addition to local convective flow, the layer may participate in convective flow patterns between layers or "aquifers" in the waste, with channels appearing and disappearing as material is yielded by shearing and then reacquires elastic solid-like properties from aging. 
TO: Tom Bander

cc: T. B. McCall

SUBJECT: Location Of TC Probes for Tank C-106

References:

H-2-90342, Piping Thermocouple Tree

H-2-90344, Piping Riser Schedule

H-2-34304, Thermocouple Probe Standard

H-2-73346, Piping Waste Tank Isolation TK 241-C-106

WHC-SD-WM-TI-553, Thermocouple Status

The following is my assessment on the locations of the TC probes in tank C-106. The probes are in Riser 8 and 14 .

Figure 1 depicts the layout and side elevation of tank C-106, the location of Riser 8 and 14 are depicted. Riser 8 flange is at elev. 646.16' while Riser 14 is at elevation $645.5^{\prime}$. The tank center bottom elevation is specified at 607'-0". The length of the TC probes are noted on Figure 1, these lengths were determined from the references noted above. The length of the R-8 TC probe is $37^{\prime}-113 / 4 "$, while the $\mathrm{R}-14$ probe is $37^{\prime}-6 "$ ":

The local tank bottom at a riser is determined from the following equation;

$$
\left.b=r-\sqrt{(} r^{2}-\frac{c^{2}}{4}\right)
$$

where :

$\mathrm{b}=$ distance above tank bottom center, ft.,

$r=$ radius of tank bottom, $569.59 \mathrm{ft}$,

$C=$ chord length at riser, $34^{\prime}-1$ 1/2" for R-8 and $15^{\prime}-7^{\prime \prime}$ for R-14. 
. therefore;

$$
\begin{aligned}
& b_{8}=1.0 \mathrm{ft} \text {. and, } \\
& b_{14}=2.5 \text { inches }
\end{aligned}
$$

The distance between the tip of the TC probe and the local tank bottom is then determined by; and;

$$
d_{8}=646.16^{\prime}-37.979^{\prime}-607^{\prime}+1^{\prime}=0.18^{\prime}=2-1 / 8^{\prime \prime}
$$

$$
\mathrm{d}_{14}=645.5^{\prime}-37.5^{\prime}-607^{\prime}+2.5 / 12=0.79^{\prime}=9.5^{\prime \prime}
$$

The design of the R-8 TC probe is depicted on $\mathrm{H}-2-90342$, the location of the tip of TC \#1 is 1-1/4" above the bottom of the probe. The design of the R-14 TC probe is depicted on $\mathrm{H}-2-34304$, the location of the tip of TC \# 1 is 4 " above the bottom of the probe. These dimensions locate the TC's above the tank bottom. Riser R-8 and R-14 TC's are spaced every $2^{\prime}$ above TC \#1.

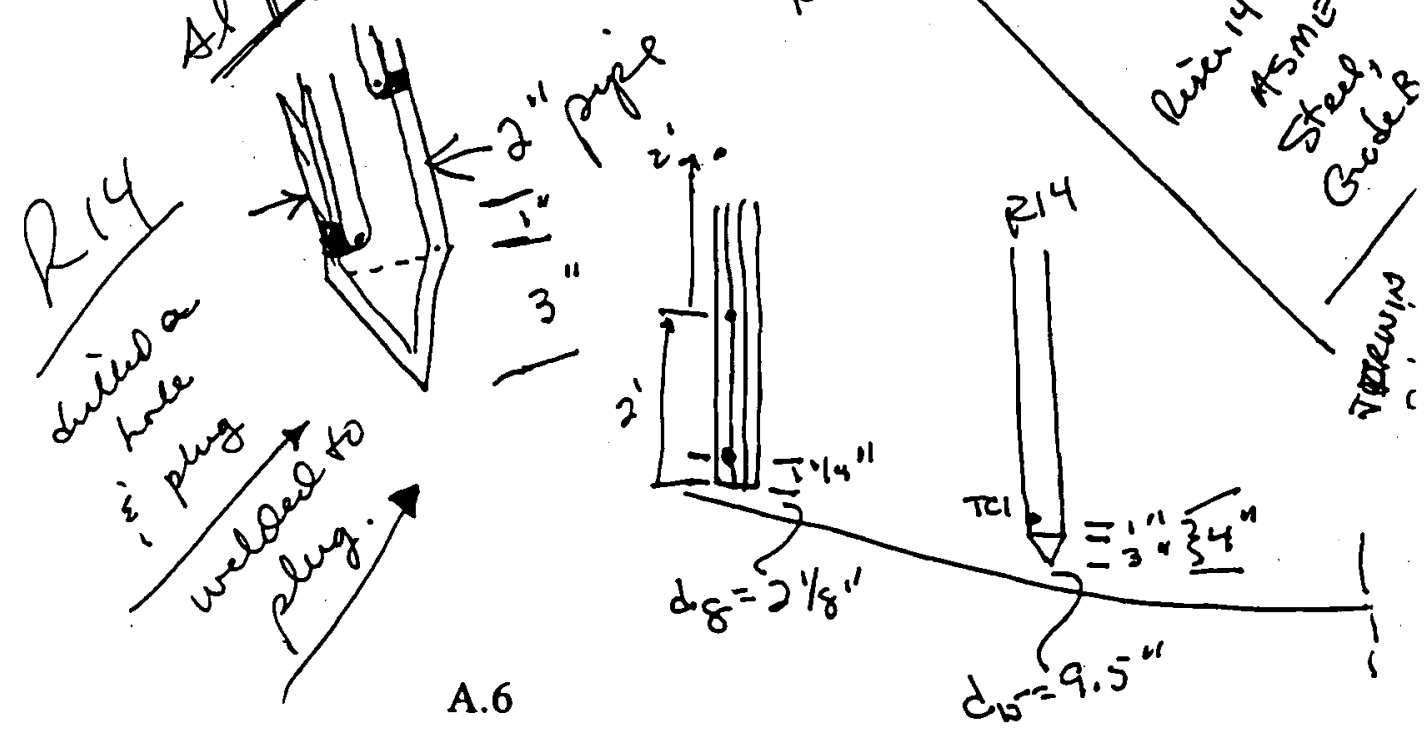




\section{C-106 TANK}

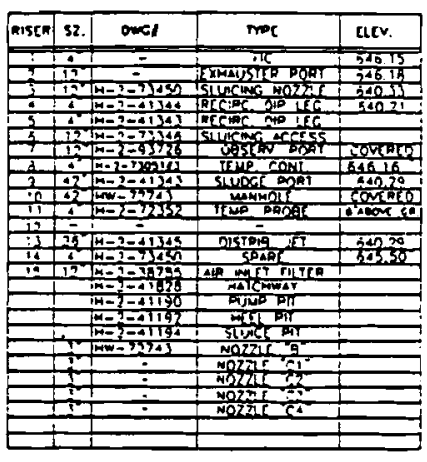

- includes o 6. SPacef

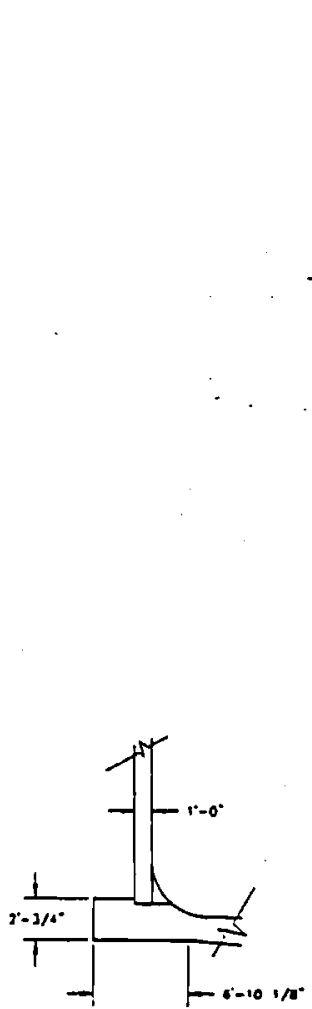

$1943-44$

$533.000 \mathrm{GAL}$
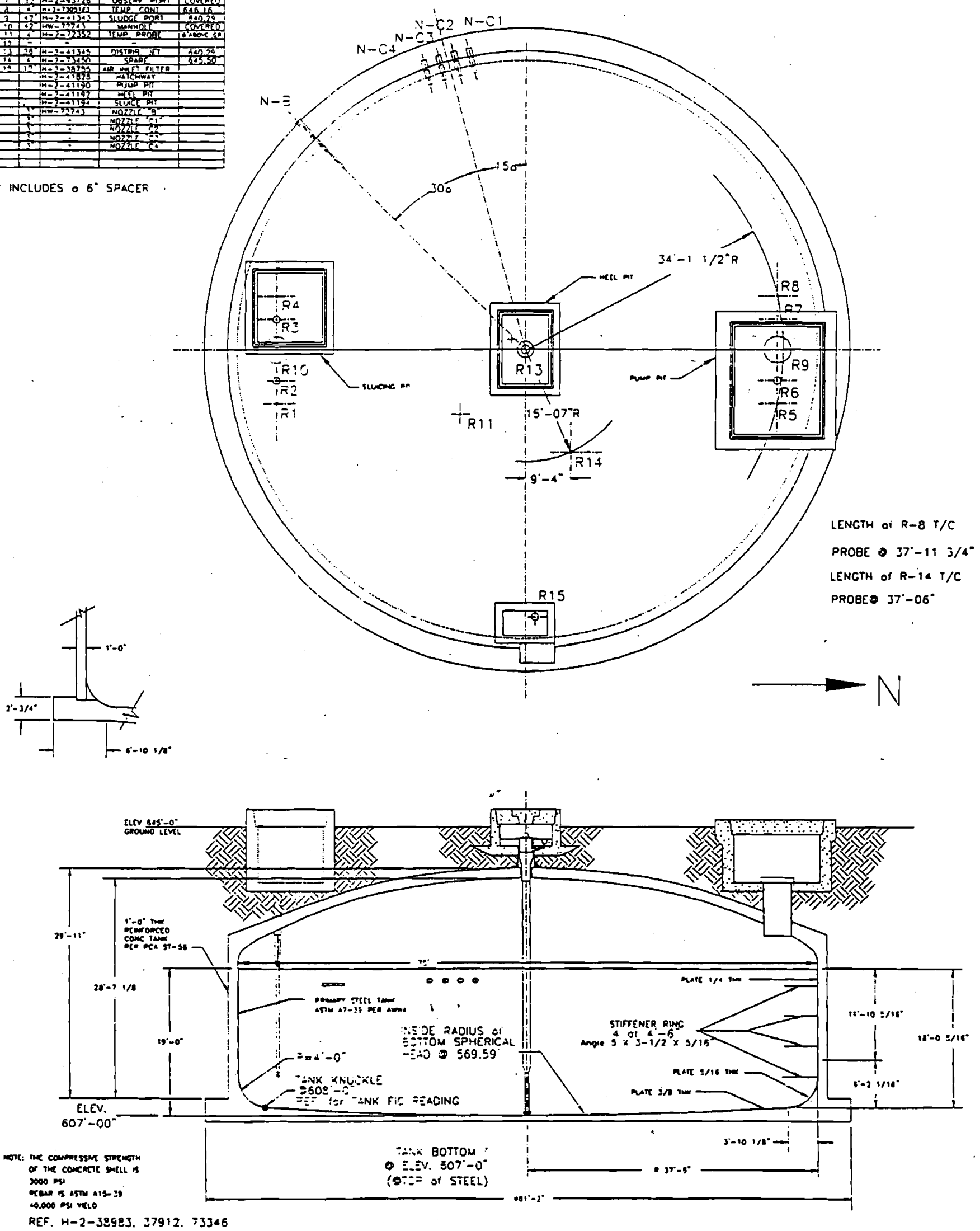


\begin{tabular}{|c|c|c|c|c|}
\hline epared By: & bLEyla & Date: ptug94 & Project: & C-106 Riser $14 \xi R_{i 2 e n} 8$ TC Thees \\
\hline ile/Subject: & & & & - per Bander / Eewin meno \\
\hline
\end{tabular}
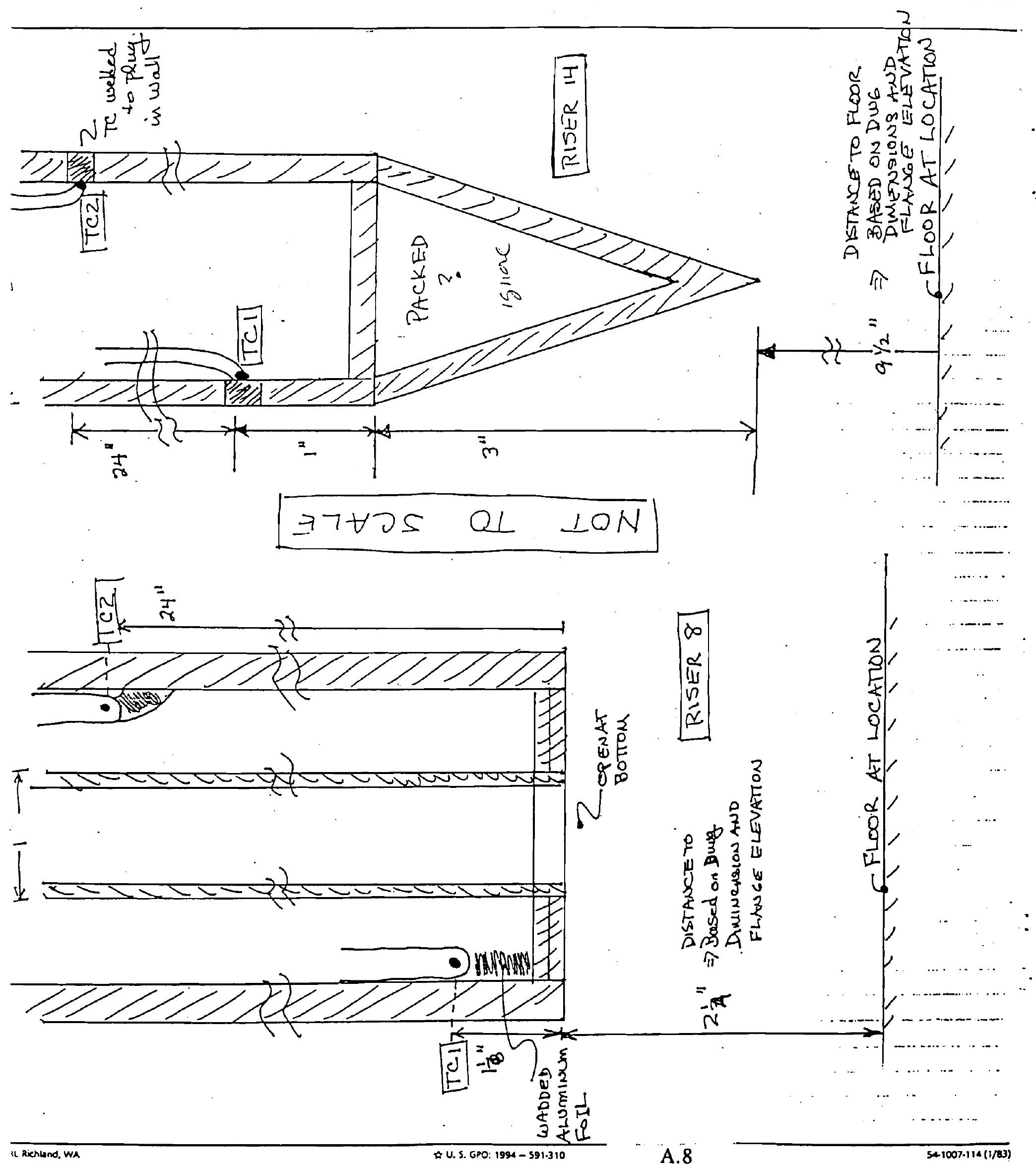
PNL-10514

UC-721

\section{Distribution}

No. of

Copies

2 DOE Office of Scientific and Technical Information

Onsite

5 U.S. Department of Energy Richland Operations Office

R.F. Christensen

G.W. Rosenwald

Public Reading Room

RL Docket File (2)

10 Westinghouse Hanford Company

T.J. Bander

R.J. Cash

E.J. (Ed) Lipke

T.B. McCall

D.M. Ogden

O.S. Wang (5)
No. of

Copies

19 Pacific Northwest Laboratory

Z.I. Antoniak

R.M. Bean

G.H. Beeman

L.L. Eyler (5)

J.A. Fort

B.M. Johnson

H.C. Reid

C.W. Stewart

P.D. Whitney

Publishing Coordination

Technical Report Files (5) 
. 